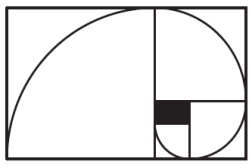

\title{
Lectures on singular stochastic
}

\section{PDEs}

\section{Massimiliano Gubinelli}

\section{Nicolas Perkowski}

\begin{abstract}
These are the notes for a course at the 18th Brazilian School of Probability held from August 3rd to 9th, 2014 in Mambucaba. The aim of the course is to introduce the basic problems of non-linear PDEs with stochastic and irregular terms. We explain how it is possible to handle them using two main techniques: the notion of energy solutions in [Gonçalves and Jara, Arch. Ration. Mech. Anal., 2014] and [Gubinelli and Jara, Stoch. Partial Diff. Equations: Analysis and Computations, 2013], and that of paracontrolled distributions, recently introduced in [Gubinelli, Imkeller, and Perkowski, Forum Math. Pi, 2015]. In order to maintain a link with physical intuitions, we motivate such singular SPDEs via a homogenization result for a diffusion in a random potential.
\end{abstract}





\section{Contents}

1 Introduction 5

2 Energy solutions 9

2.1 Distributions . . . . . . . . . . . . . . . . 999

2.2 The Stochastic Burgers equation . . . . . . . . . . . 11

2.3 The Ornstein-Uhlenbeck process . . . . . . . . . . . . . . 12

2.4 Gaussian computations . . . . . . . . . . . . . . . . 19

2.5 The Itô trick . . . . . . . . . . . . . . . . . . . . . . 27

2.6 Controlled distributions . . . . . . . . . . . . . . . . . . . . 33

2.7 Existence of solutions . . . . . . . . . . . . . . 34

$\begin{array}{llr}3 & \text { Besov spaces } & 37\end{array}$

4 Diffusion in a random environment 44

4.1 The 2d generalized parabolic Anderson model . . . . . . . . 50

4.2 More singular problems . . . . . . . . . . . . . . 52

4.3 Hairer's regularity structures . . . . . . . . . . . . . . 54

5 The paracontrolled PAM $\mathbf{5 5}$

5.1 The paraproduct and the resonant term . . . . . . . . 56

5.2 Commutator estimates and paralinearization . . . . . . . 59

5.3 Paracontrolled distributions . . . . . . . . . . . . . 66

5.4 Fixpoint ...................... 70

5.5 Renormalization ................ . . 71

5.6 Construction of the extended data . . . . . . . . . . 76

6 The stochastic Burgers equation $\quad 81$

6.1 Structure of the solution . . . . . . . . . . . . . . . . . 82

6.2 Paracontrolled solution . . . . . . . . . . . . 83

$\begin{array}{lr}\text { Bibliography } & 87\end{array}$ 



\section{Chapter 1}

\section{Introduction}

The aim of these lectures is to explain how to apply controlled path ideas [12] to solve basic problems in singular stochastic parabolic equations. The hope is that the insight gained by doing so can inspire new applications or the construction of other more powerful tools to analyze a wider class of problems.

To understand the origin of such singular equations, we have chosen to present the example of a homogenization problem for a singular potential in a linear parabolic equation. This point of view has the added benefit that it allows us to track back the renormalization needed to handle the singularities as effects living on other scales than those of interest. The basic problem is that of having to handle effects of the microscopic scales and their interaction through non-linearities on the macroscopic behaviour of the solution.

Mathematically, this problem translates into the attempt of making Schwartz's theory of distributions coexist with non-linear operations which are notoriously not continuous in the usual topologies on distributions. This is a very old problem of analysis and has been widely studied. The additional input which is not present in the usual approaches is that the singularities which force us to treat the problem in the setting of Schwartz's distributions are of a stochastic nature. So we dispose of two handles on the problem: the analytical one and the probabilistic one. The right mix of the two will provide an effective solution to a wide class of problems.

A first and deep understanding of these problems has been obtained starting from the late '90s by T. Lyons [25], who introduced a theory of rough paths in order to settle the conflict of topology and non-linearity in the context of driven differential equations, or more generally in the context of the non-linear analysis of time-varying signals. Nowadays there are many expositions of this theory $[27,9,26,8]$ and we refer the reader to the literature for more details. 
In $[12,13]$, the notion of controlled paths has been introduced in order to extend the applicability of the rough path ideas to a larger class of problems that are not necessarily related to the integration of ODEs but which still retain the one-dimensional nature of the directions in which the irregularity manifests itself. The controlled path approach has been used to make sense of the evolution of irregular objects such as vortex filaments and certain SPDEs. Later Hairer understood how to apply these ideas to the long standing problem of the Kardar-Parisi-Zhang equation [18], and his insights prompted the researchers to try more ambitious approaches to extend rough paths to a multidimensional setting.

In [14], in collaboration with P. Imkeller, we introduced a notion of paracontrolled distributions which is suitable to handle a wide class of SPDEs which were well out of reach with previously known methods. Paracontrolled distributions can be understood as an extension of controlled paths to a multidimensional setting, and they are based on new combinations of basic tools from harmonic analysis.

At the same time, Hairer managed to devise a vast generalization of the basic construction of controlled rough paths in the multidimensional and distributional setting, which he called the theory of regularity structures [19] and which subsumes standard analysis based on Hölder spaces and controlled rough path theory but goes well beyond that. Just a few days after the lectures in Mambucaba took place, it was announced that Martin Hairer was awarded a Fields Medal for his work on SPDEs and in particular for his theory of regularity structures [19] as a tool for dealing with singular SPDEs. This prize witnesses the exciting period we are experiencing: we now understand sound lines of attack to long standing problems, and there are countless opportunities to apply similar ideas to new problems.

The plan of the lectures is the following. We start by discussing energy solutions $[10,11,15]$ of the stationary stochastic Burgers equation (one of the avatars of the Kardar-Parisi-Zhang equation). ${ }^{1}$ Energy solutions have the advantage of being relatively easy to handle and of being based on tools that are familiar to probabilists. On the other side, they only apply in the specific example of the stochastic Burgers equation in equilibrium, and here we will only focus on the existence but not on the uniqueness of energy solutions. Starting our lectures in this way will allow us to introduce the reader to SPDEs in a progressive manner, and also to introduce Gaussian tools on the way (Wick products, hypercontractivity) and to present some of the basic phenomena that appear when dealing with singular SPDEs. Next we set up the analytical tools we need in the rest of the lectures: Besov spaces and some basic harmonic analysis based on

\footnotetext{
${ }^{1}$ The paper [11] is the revised published version of [10]. We would like to cite them together to acknowledge that the notion of energy solutions historically predates that of Hairer in [18].
} 
the Littlewood-Paley decomposition of distributions. In order to motivate the reader and to provide a physical ground for the intuition to stand on, we then discuss a homogenization problem for the linear heat equation with random potential which describes diffusion in a random environment. This will allow us to derive the need for the weak topologies we shall use and for irregular objects like the white noise from first principles and "concrete" applications. The homogenization problem also allows us to see that there are naturally appearing renormalization effects and to keep track of their mathematical meaning. Starting from these problems we introduce the two-dimensional parabolic Anderson model, the simplest SPDE in which most of the features of more difficult problems are already present, and we explain how to use paraproducts and the paracontrolled ansatz in order to keep the non-linear effect of the singular data under control. Then we return to the stochastic Burgers equation and show how to apply paracontrolled distributions in order to obtain the existence and uniqueness of solutions also in the non-stationary case. effect of the singular data under control. Then we return to the stochastic Burgers equation and show how to apply paracontrolled distributions in order to obtain the existence and uniqueness of solutions also in the non-stationary case. effect of the singular data under control. Then we return to the stochastic Burgers equation and show how to apply paracontrolled distributions in order to obtain the existence and uniqueness of solutions also in the non-stationary case.

Acknowledgements. The authors would like to thank the two anonymous referees for the careful reading and the manifold suggestions which helped up to greatly improve the manuscript. We would also like to thank the organizers of the Brazilian Summer Schools in Probability for the invitation and the researchers who attended the meeting for the wonderful atmosphere.

The main part of the research was carried out while N. P. was employed by Université Paris Dauphine. N. P. was supported by the Fondation Sciences Mathématiques de Paris (FSMP) and by a public grant overseen by the French National Research Agency (ANR) as part of the "Investissements d'Avenir" program (reference: ANR-10-LABX-0098).

Conventions and notations. We write $a \lesssim b$ if there exists a constant $C>0$, independent of the variables under consideration, such that $a \leqslant C b$. Similarly we define $\gtrsim$. We write $a \simeq b$ if $a \lesssim b$ and $b \lesssim a$. If we want to emphasize the dependence of $C$ on the variable $x$, then we write $a(x) \lesssim_{x} b(x)$.

If $a$ is a complex number, we write $a^{*}$ for its complex conjugate.

If $i$ and $j$ are index variables of Littlewood-Paley blocks (to be defined below), then $i \lesssim j$ is to be interpreted as $2^{i} \lesssim 2^{j}$, and similarly for $\simeq$ and 
$\lesssim$. In other words, $i \lesssim j$ means $i \leqslant j+N$ for some fixed $N \in \mathbb{N}$ that does not depend on $i$ or $j$.

We use standard multi-index notation: for $\mu \in \mathbb{N}_{0}^{d}$ we write $|\mu|=$ $\mu_{1}+\ldots+\mu_{d}$ and $\partial^{\mu}=\partial^{|\mu|} / \partial_{x_{1}}^{\mu_{1}} \ldots \partial_{x_{d}}^{\mu_{d}}$, as well as $x^{\mu}=x_{1}^{\mu_{1}} \cdot \ldots \cdot x_{d}^{\mu_{d}}$ for $x \in \mathbb{R}^{d}$.

For $\alpha>0$ we write $C_{b}^{\alpha}$ for the bounded functions $F: \mathbb{R} \rightarrow \mathbb{R}$ which are $\lfloor\alpha\rfloor$ times continuously differentiable with bounded and $(\alpha-\lfloor\alpha\rfloor)$-Hölder continuous derivatives of order $\lfloor\alpha\rfloor$, equipped with the norm

$$
\|F\|_{C_{b}^{\alpha}}=\sup _{\mu: 0 \leq|\mu| \leq\lfloor\alpha\rfloor}\left\|\partial^{\mu} F\right\|_{L^{\infty}}+\sup _{\mu:|\mu|=\lfloor\alpha\rfloor} \sup _{x \neq y} \frac{\left|\partial^{\mu} F(x)-\partial^{\mu} F(y)\right|}{|x-y|^{\alpha-\lfloor\alpha\rfloor}} .
$$

If we write $u \in \mathscr{C}^{\alpha-}$, then that means that $u$ is in $\mathscr{C}^{\alpha-\varepsilon}$ for all $\varepsilon>0$. The $\mathscr{C}^{\alpha}$ spaces will be defined below.

If $\mathbb{X}$ is a Banach space with norm $\|\cdot\|_{\mathbb{X}}$ and if $T>0$, then we define $C \mathbb{X}$ and $C_{T} \mathbb{X}$ as the spaces of continuous functions from $[0, \infty)$ respectively $[0, T]$ to $\mathbb{X}$, and $C_{T} \mathbb{X}$ is equipped with the supremum norm $\|\cdot\|_{C_{T} \mathbb{X}}$. If $\alpha \in(0,1)$ then we write $C^{\alpha} \mathbb{X}$ for the functions in $C \mathbb{X}$ that are $\alpha$-Hölder continuous on every interval $[0, T]$, and we write

$$
\|f\|_{C_{T}^{\alpha \mathbb{X}}}=\sup _{0 \leqslant s<t \leqslant T} \frac{\|f(t)-f(s)\|}{|t-s|^{\alpha}} .
$$




\section{Chapter 2}

\section{Energy solutions}

The first issue one encounters when dealing with singular SPDEs is the ill-posed character of the equation, even in a weak sense. Typically, the equation features some non-linearity that does not make sense in the natural spaces where solutions live and one has to provide a suitable smaller space in which it is possible to give an appropriate interpretation to "ambiguous quantities" that appear in the equation.

Energy solutions $[11,15]$ are a relatively simple tool in order to come up with well-defined non-linearities. Moreover, proving existence of energy solutions or even convergence to energy solutions is usually a quite simple problem, at least compared to the other approaches like paracontrolled solutions or regularity structures, where already existence requires quite a large amount of computations but where uniqueness can be established quite easily afterwards. The main drawback is that we lack of general uniqueness results for energy solutions. Only very recently, after the completion of these notes, we were able to prove that energy solutions for the stationary stochastic Burgers equation are unique. This topic will not be touched upon here. The interested reader can find the details in the preprint [17].

\subsection{Distributions}

We will need to use distributions defined on the $d$-dimensional torus $\mathbb{T}^{d}$ where $\mathbb{T}=\mathbb{R} /(2 \pi \mathbb{Z})$. We collect here some basic results and definitions. The space of distributions $\mathscr{S}^{\prime}=\mathscr{S}^{\prime}\left(\mathbb{T}^{d}\right)$ is the set of linear maps $f$ from $\mathscr{S}=C^{\infty}\left(\mathbb{T}^{d}, \mathbb{C}\right)$ to $\mathbb{C}$, such that there exist $k \in \mathbb{N}$ and $C>0$ with

$$
|\langle f, \varphi\rangle|:=|f(\varphi)| \leqslant C \sup _{|\mu| \leqslant k}\left\|\partial^{\mu} \varphi\right\|_{L^{\infty}\left(\mathbb{T}^{d}\right)}
$$


for all $\varphi \in \mathscr{S}$.

Example 1. Clearly $L^{p}=L^{p}\left(\mathbb{T}^{d}\right) \subset \mathscr{S}^{\prime}$ for all $p \geqslant 1$, and more generally the space of finite signed measures on $\left(\mathbb{T}^{d}, \mathscr{B}\left(\mathbb{T}^{d}\right)\right)$ is contained in $\mathscr{S}^{\prime}$. Another example of a distribution is $\varphi \mapsto \partial^{\mu} \varphi(x)$ for $\mu \in \mathbb{N}_{0}^{d}$ and $x \in \mathbb{T}$.

In particular, the Fourier transform $\mathscr{F} f: \mathbb{Z}^{d} \rightarrow \mathbb{C}$,

$$
\mathscr{F} f(k)=\hat{f}(k)=\left\langle f, e_{k}\right\rangle,
$$

with $e_{k}=e^{-i\langle k, \cdot\rangle} /(2 \pi)^{d / 2}$, is defined for all $f \in \mathscr{S}^{\prime}$, and it satisfies $|\mathscr{F} f(k)| \leqslant|P(k)|$ for a suitable polynomial $P$. Conversely, if $(g(k))_{k \in \mathbb{Z}^{d}}$ is at most of polynomial growth, then its inverse Fourier transform

$$
\mathscr{F}^{-1} g=\sum_{k \in \mathbb{Z}^{d}} g(k) e_{k}^{*}
$$

defines a distribution (here $e_{k}^{*}=e^{i\langle k, \cdot\rangle} /(2 \pi)^{d / 2}$ is the complex conjugate of $\left.e_{k}\right)$.

Exercise 1. Show that the Fourier transform of $\varphi \in \mathscr{S}$ decays faster than any rational function (we say that it is of rapid decay). Combine this with the fact that $\mathscr{F}$ defines a bijection from $L^{2}\left(\mathbb{T}^{d}\right)$ to $\ell^{2}\left(\mathbb{Z}^{d}\right)$ with inverse $\mathscr{F}^{-1}$ to show that $\mathscr{F}^{-1} \mathscr{F} f=f$ for all $f \in \mathscr{S}^{\prime}$ and $\mathscr{F} \mathscr{F}^{-1} g=g$ for all $g$ of polynomial growth. Extend the Parseval formula

$$
\left\langle f, \varphi^{*}\right\rangle_{L^{2}\left(\mathbb{T}^{d}\right)}=\int_{\mathbb{T}^{d}} f(x) \varphi(x)^{*} \mathrm{~d} x=\sum_{k} \hat{f}(k) \hat{\varphi}(k)^{*}
$$

from $f, \varphi \in L^{2}\left(\mathbb{T}^{d}\right)$ to $f \in \mathscr{S}^{\prime}$ and $\varphi \in \mathscr{S}$.

Exercise 2. Fix a complete probability space $(\Omega, \mathcal{F}, \mathbb{P})$. On that space let $\xi$ be a spatial white noise on $\mathbb{T}^{d}$, i.e. $\xi$ is a centered Gaussian process indexed by $L^{2}\left(\mathbb{T}^{d}\right)$, with covariance

$$
\mathbb{E}[\xi(f) \xi(g)]=\int_{\mathbb{T}^{d}} f(x) g(x) \mathrm{d} x .
$$

Show that there exists $\tilde{\xi}$ with $\mathbb{P}(\tilde{\xi}(f)=\xi(f))=1$ for all $f \in L^{2}$, such that $\tilde{\xi}(\omega) \in \mathscr{S}^{\prime}$ for all $\omega \in \Omega$.

Hint: Show that $\mathbb{E}\left[\sum_{k \in \mathbb{Z}^{d}} \exp \left(\lambda\left|\xi\left(e_{k}\right)\right|^{2}\right) /\left(1+|k|^{d+1}\right)\right]<\infty$ for some suitable $\lambda>0$.

Linear maps on $\mathscr{S}^{\prime}$ can be defined by duality: if $A: \mathscr{S} \rightarrow \mathscr{S}$ is such that for all $k \in \mathbb{N}$ there exists $n \in \mathbb{N}$ and $C>0$ with $\sup _{|\mu| \leqslant k}\left\|\partial^{\mu}(A \varphi)\right\|_{L^{\infty}} \leqslant C \sup _{|\mu| \leqslant n}\left\|\partial^{\mu} \varphi\right\|_{L^{\infty}}$, then we set $\left\langle{ }^{t} A f, \varphi\right\rangle=$ $\langle f, A \varphi\rangle$. Differential operators are defined by $\left\langle\partial^{\mu} f, \varphi\right\rangle=(-1)^{|\mu|}\left\langle f, \partial^{\mu} \varphi\right\rangle$. If $\varphi: \mathbb{Z}^{d} \rightarrow \mathbb{C}$ grows at most polynomially, then it defines a Fourier multiplier

$$
\varphi(\mathrm{D}): \mathscr{S}^{\prime} \rightarrow \mathscr{S}^{\prime}, \quad \varphi(\mathrm{D}) f=\mathscr{F}^{-1}(\varphi \mathscr{F} f) .
$$


Exercise 3. Use the Fourier inversion formula of Exercise 1 to show that for $f \in \mathscr{S}^{\prime}, \varphi \in \mathscr{S}$ and for $u, v: \mathbb{Z}^{d} \rightarrow \mathbb{C}$ with $u$ of polynomial growth and $v$ of rapid decay

$$
\mathscr{F}(f \varphi)(k)=(2 \pi)^{-d / 2} \sum_{\ell} \hat{f}(k-\ell) \hat{\varphi}(\ell)
$$

and

$$
\mathscr{F}^{-1}(u v)(x)=(2 \pi)^{d / 2}\left\langle\mathscr{F}^{-1} u,\left(\mathscr{F}^{-1} v\right)(x-\cdot)\right\rangle
$$

\subsection{The Stochastic Burgers equation}

Our aim here is to motivate the ideas at the base of the notion of energy solutions. We will not insist on a detailed formulation of all the available results. The reader can always refer to the original paper [15] for missing details. Applications to the large scale behavior of particle systems are studied in [11].

We will study the case of the stochastic Burgers equation on the torus $\mathbb{T}$. The solution of the stochastic Burgers equation is the derivative of the solution of the Kardar-Parisi-Zhang equation, a universal model for the fluctuations in random interface growth which has been at the center of several spectacular results of the past years. Excellent surveys on the KPZ equation and related areas are [6, 28, 29].

The unknown $u: \mathbb{R}_{+} \times \mathbb{T} \rightarrow \mathbb{R}$ should satisfy

$$
\partial_{t} u=\Delta u+\partial_{x} u^{2}+\partial_{x} \xi,
$$

where $\xi: \mathbb{R}_{+} \times \mathbb{T} \rightarrow \mathbb{R}$ is a space-time white noise defined on a given probability space $(\Omega, \mathcal{F}, \mathbb{P})$ fixed once and for all. That is, $\xi$ is a centered Gaussian process indexed by $L^{2}\left(\mathbb{R}_{+} \times \mathbb{T}\right)$ with covariance

$$
\mathbb{E}[\xi(f) \xi(g)]=\int_{\mathbb{R}_{+} \times \mathbb{T}} f(t, x) g(t, x) \mathrm{d} t \mathrm{~d} x .
$$

The equation has to be understood as a relation for processes which are distributions in space with sufficiently regular time dependence. In particular, if we test the above relation with $\varphi \in \mathscr{S}:=\mathscr{S}(\mathbb{T}):=C^{\infty}(\mathbb{T})$, denote with $u_{t}(\varphi)$ the pairing of the distribution $u(t, \cdot)$ with $\varphi$, and integrate in time over the interval $[0, t]$, we formally get

$$
u_{t}(\varphi)=u_{0}(\varphi)+\int_{0}^{t} u_{s}(\Delta \varphi) \mathrm{d} s-\int_{0}^{t}\left\langle u_{s}^{2}, \partial_{x} \varphi\right\rangle \mathrm{d} s-\int_{0}^{t} \xi_{s}\left(\partial_{x} \varphi\right) \mathrm{d} s .
$$


Let us discuss the various terms in this equation. In order to make sense of $u_{t}(\varphi)$ and $\int_{0}^{t} u_{s}(\Delta \varphi) \mathrm{d} s$, it is enough to assume that for all $\varphi \in \mathscr{S}$ the mapping $(t, \omega) \mapsto u_{t}(\varphi)(\omega)$ is a stochastic process with continuous trajectories. Next, if we denote $M_{t}(\varphi)=-\int_{0}^{t} \xi_{s}\left(\partial_{x} \varphi\right) \mathrm{d} s$ then, at least by a formal computation, we have that $\left(M_{t}(\varphi)\right)_{t \geqslant 0, \varphi \in \mathscr{S}}$ is a Gaussian random field with covariance

$$
\mathbb{E}\left[M_{t}(\varphi) M_{s}(\psi)\right]=(t \wedge s)\left\langle\partial_{x} \varphi, \partial_{x} \psi\right\rangle_{L^{2}(\mathbb{T})}
$$

In particular, for every $\varphi \in \mathscr{S}$ the stochastic process $\left(M_{t}(\varphi)\right)_{t \geqslant 0}$ is a Brownian motion with covariance

$$
\|\varphi\|_{H^{1}(\mathbb{T})}^{2}:=\left\langle\partial_{x} \varphi, \partial_{x} \varphi\right\rangle_{L^{2}(\mathbb{T})}
$$

We will use this fact to have a rigorous interpretation of the white noise $\xi$ appearing in the equation. Here we used the notation $M$ in order to stress the fact that $M_{t}(\varphi)$ is a martingale in its natural filtration and more generally in the filtration $\mathcal{F}_{t}=\sigma\left(M_{s}(\varphi): s \leqslant t, \varphi \in H^{1}(\mathbb{T})\right), t \geqslant 0$.

The most difficult term is of course the nonlinear one: $\int_{0}^{t}\left\langle u_{s}^{2}, \partial_{x} \varphi\right\rangle \mathrm{d} s$. In order to define it, we need to square the distribution $u_{t}$, an operation which in general can be quite dangerous. A natural approach would be to define it as the limit of some regularizations. For example, if $\rho: \mathbb{R} \rightarrow \mathbb{R}_{+}$ is a compactly supported $C^{\infty}$ function such that $\int_{\mathbb{R}} \rho(x) \mathrm{d} x=1$, and we set $\rho_{\varepsilon}(\cdot)=\rho(\cdot / \varepsilon) / \varepsilon$, then we can set $\mathcal{N}_{t, \varepsilon}(u)(x)=\int_{0}^{t}\left(\left(\rho_{\varepsilon} * u_{s}\right)(x)\right)^{2} \mathrm{~d} s$ and define $\mathcal{N}_{t}(u)=\lim _{\varepsilon \rightarrow 0} \mathcal{N}_{t, \varepsilon}(u)$ whenever the limit exists in $\mathscr{S}^{\prime}:=\mathscr{S}^{\prime}(\mathbb{T})$, the space of distributions on $\mathbb{T}$. Then the question arises which properties $u$ should have for this convergence to occur.

\subsection{The Ornstein-Uhlenbeck process}

Let us simplify the problem and start by studying the linearized equation obtained by neglecting the non-linear term. Let $X$ be a solution to

$$
X_{t}(\varphi)=X_{0}(\varphi)+\int_{0}^{t} X_{s}(\Delta \varphi) \mathrm{d} s+M_{t}(\varphi)
$$

for all $t \geqslant 0$ and $\varphi \in \mathscr{S}$. This equation has at most one solution (for fixed $X_{0}$ ). Indeed, the difference $D$ between two solutions should satisfy $D_{t}(\varphi)=\int_{0}^{t} D_{s}(\Delta \varphi) \mathrm{d} s$, which means that $D$ is a distributional solution to the heat equation. Taking $\varphi(x)=e_{k}(x)$, where

$$
e_{k}(x):=\exp (-i k x) / \sqrt{2 \pi}, \quad k \in \mathbb{Z}
$$


we get $D_{t}\left(e_{k}\right)=-k^{2} \int_{0}^{t} D_{s}\left(e_{k}\right) \mathrm{d} s$ and then by Gronwall's inequality $D_{t}\left(e_{k}\right)=0$ for all $t \geqslant 0$. This easily implies that $D_{t}=0$ in $\mathscr{S}^{\prime}$ for all $t \geqslant 0$.

To obtain the existence of a solution, observe that

$$
X_{t}\left(e_{k}\right)=X_{0}\left(e_{k}\right)-k^{2} \int_{0}^{t} X_{s}\left(e_{k}\right) \mathrm{d} s+M_{t}\left(e_{k}\right)
$$

and that $M_{t}\left(e_{0}\right)=0$, while for all $k \neq 0$ the process $\beta_{t}(k)=M_{t}\left(e_{k}\right) /(-i k)$ is a complex valued Brownian motion (i.e. real and imaginary part are independent Brownian motions with the same variance). The covariance of $\beta$ is given by

$$
\mathbb{E}\left[\beta_{t}(k) \beta_{s}(m)\right]=(t \wedge s) \delta_{k+m=0}
$$

and moreover $\beta_{t}(k)^{*}=\beta_{t}(-k)$ for all $k \neq 0$ (where $\cdot^{*}$ denotes complex conjugation), as well as $\beta_{t}(0)=0$. In other words, $\left(X_{t}\left(e_{k}\right)\right)$ is a complexvalued Ornstein-Uhlenbeck process ([23], Example 5.6.8) which solves a linear one-dimensional SDE and has an explicit representation given by the variation of constants formula

$$
X_{t}\left(e_{k}\right)=e^{-k^{2} t} X_{0}\left(e_{k}\right)-i k \int_{0}^{t} e^{-k^{2}(t-s)} \mathrm{d}_{s} \beta_{s}(k) .
$$

This is enough to determine $X_{t}(\varphi)$ for all $t \geqslant 0$ and $\varphi \in \mathscr{S}$.

Exercise 4. Show that $\left(X_{t}\left(e_{k}\right): t \in \mathbb{R}_{+}, k \in \mathbb{Z}\right)$ is a complex Gaussian random field, that is for all $n \in \mathbb{N}$, for all $t_{1}, \ldots, t_{n} \in \mathbb{R}_{+}, k_{1}, \ldots, k_{n} \in \mathbb{Z}$, the vector

$$
\left(\operatorname{Re}\left(X_{t_{1}}\left(e_{k_{1}}\right)\right), \ldots, \operatorname{Re}\left(X_{t_{n}}\left(e_{k_{n}}\right)\right), \operatorname{Im}\left(X_{t_{1}}\left(e_{k_{1}}\right)\right), \ldots, \operatorname{Im}\left(X_{t_{n}}\left(e_{k_{n}}\right)\right)\right)
$$

is multivariate Gaussian. Show that $X$ has mean $\mathbb{E}\left[X_{t}\left(e_{k}\right)\right]=e^{-k^{2} t} X_{0}\left(e_{k}\right)$ and covariance

$\mathbb{E}\left[\left(X_{t}\left(e_{k}\right)-\mathbb{E}\left[X_{t}\left(e_{k}\right)\right]\right)\left(X_{s}\left(e_{m}\right)-\mathbb{E}\left[X_{s}\left(e_{m}\right)\right]\right)\right]=k^{2} \delta_{k+m=0} \int_{0}^{t \wedge s} e^{-k^{2}(t-r)-k^{2}(s-r)} \mathrm{d} r$ as well as

$\mathbb{E}\left[\left(X_{t}\left(e_{k}\right)-\mathbb{E}\left[X_{t}\left(e_{k}\right)\right]\right)\left(X_{s}\left(e_{m}\right)-\mathbb{E}\left[X_{s}\left(e_{m}\right)\right]\right)^{*}\right]=k^{2} \delta_{k=m} \int_{0}^{t \wedge s} e^{-k^{2}(t-r)-k^{2}(s-r)} \mathrm{d} r$.

In particular,

$$
\mathbb{E}\left[\left|X_{t}\left(e_{k}\right)-\mathbb{E}\left[X_{t}\left(e_{k}\right)\right]\right|^{2}\right]=\frac{1-e^{-2 k^{2} t}}{2} .
$$

Next we examine the Sobolev regularity of $X$. For this purpose, we need the following definition. 
Definition 1. Let $\alpha \in \mathbb{R}$. Then the Sobolev space $H^{\alpha}$ is defined as

$$
H^{\alpha}:=H^{\alpha}(\mathbb{T}):=\left\{\rho \in \mathscr{S}^{\prime}:\|\rho\|_{H^{\alpha}}^{2}:=\sum_{k \in \mathbb{Z}}\left(1+|k|^{2}\right)^{\alpha}\left|\rho\left(e_{k}\right)\right|^{2}<\infty\right\} .
$$

We also write $C H^{\alpha}$ for the space of continuous functions from $\mathbb{R}_{+}$to $H^{\alpha}$.

Lemma 1. Let $\gamma \leqslant-1 / 2$ and assume that $X_{0} \in H^{\gamma}$. Then almost surely $X \in C H^{\gamma-}$.

Proof. Let $\alpha=\gamma-\varepsilon$ and consider

$$
\left\|X_{t}-X_{s}\right\|_{H^{\alpha}}^{2}=\sum_{k \in \mathbb{Z}}\left(1+|k|^{2}\right)^{\alpha}\left|X_{t}\left(e_{k}\right)-X_{s}\left(e_{k}\right)\right|^{2} .
$$

Let us estimate the $L^{2 p}(\Omega)$ norm of this quantity for $p \in \mathbb{N}$ by writing

$$
\mathbb{E}\left\|X_{t}-X_{s}\right\|_{H^{\alpha}}^{2 p}=\sum_{k_{1}, \ldots, k_{p} \in \mathbb{Z}} \prod_{i=1}^{p}\left(1+\left|k_{i}\right|^{2}\right)^{\alpha} \mathbb{E} \prod_{i=1}^{p}\left|X_{t}\left(e_{k_{i}}\right)-X_{s}\left(e_{k_{i}}\right)\right|^{2} .
$$

By Hölder's inequality, we get

$$
\mathbb{E}\left\|X_{t}-X_{s}\right\|_{H^{\alpha}}^{2 p} \lesssim \sum_{k_{1}, \ldots, k_{p} \in \mathbb{Z}} \prod_{i=1}^{p}\left(1+\left|k_{i}\right|^{2}\right)^{\alpha} \prod_{i=1}^{p}\left(\mathbb{E}\left|X_{t}\left(e_{k_{i}}\right)-X_{s}\left(e_{k_{i}}\right)\right|^{2 p}\right)^{1 / p}
$$

Note now that $X_{t}\left(e_{k_{i}}\right)-X_{s}\left(e_{k_{i}}\right)$ is a Gaussian random variable, so that there exists a universal constant $C_{p}$ for which

$$
\mathbb{E}\left|X_{t}\left(e_{k_{i}}\right)-X_{s}\left(e_{k_{i}}\right)\right|^{2 p} \leqslant C_{p}\left(\mathbb{E}\left|X_{t}\left(e_{k_{i}}\right)-X_{s}\left(e_{k_{i}}\right)\right|^{2}\right)^{p}
$$

Moreover,

$$
X_{t}\left(e_{k}\right)-X_{s}\left(e_{k}\right)=\left(e^{-k^{2}(t-s)}-1\right) X_{s}\left(e_{k}\right)+i k \int_{s}^{t} e^{-k^{2}(t-r)} \mathrm{d}_{r} \beta_{r}(k),
$$

leading to

$$
\begin{aligned}
& \mathbb{E}\left|X_{t}\left(e_{k}\right)-X_{s}\left(e_{k}\right)\right|^{2} \\
= & \left(e^{-k^{2}(t-s)}-1\right)^{2} \mathbb{E}\left|X_{s}\left(e_{k}\right)\right|^{2}+k^{2} \int_{s}^{t} e^{-2 k^{2}(t-r)} \mathrm{d} r \\
= & \left(e^{-k^{2}(t-s)}-1\right)^{2} e^{-2 k^{2} s}\left|X_{0}\left(e_{k}\right)\right|^{2}+\left(e^{-k^{2}(t-s)}-1\right)^{2} k^{2} \int_{0}^{s} e^{-2 k^{2}(s-r)} \mathrm{d} r \\
& +k^{2} \int_{s}^{t} e^{-2 k^{2}(t-r)} \mathrm{d} r \\
= & \left(e^{-k^{2} t}-e^{-k^{2} s}\right)^{2}\left|X_{0}\left(e_{k}\right)\right|^{2}+\frac{1}{2}\left(e^{-k^{2}(t-s)}-1\right)^{2}\left(1-e^{-2 k^{2} s}\right) \\
& +\frac{1}{2}\left(1-e^{-2 k^{2}(t-s)}\right) .
\end{aligned}
$$


For any $\kappa \in[0,1]$ and $k \neq 0$, we thus have

$$
\mathbb{E}\left|X_{t}\left(e_{k}\right)-X_{s}\left(e_{k}\right)\right|^{2} \lesssim\left(k^{2}(t-s)\right)^{\kappa}\left(\left|X_{0}\left(e_{k}\right)\right|^{2}+1\right),
$$

while for $k=0$ we have $\mathbb{E}\left|X_{t}\left(e_{0}\right)-X_{s}\left(e_{0}\right)\right|^{2}=0$. Let us introduce the notation $\mathbb{Z}_{0}=\mathbb{Z} \backslash\{0\}$. Therefore,

$$
\begin{aligned}
\mathbb{E}\left\|X_{t}-X_{s}\right\|_{H^{\alpha}}^{2 p} & \lesssim \sum_{k_{1}, \ldots, k_{p} \in \mathbb{Z}_{0}} \prod_{i=1}^{p}\left(1+\left|k_{i}\right|^{2}\right)^{\alpha} \prod_{i=1}^{p} \mathbb{E}\left|X_{t}\left(e_{k_{i}}\right)-X_{s}\left(e_{k_{i}}\right)\right|^{2} \\
& \lesssim(t-s)^{\kappa p} \sum_{k_{1}, \ldots, k_{p} \in \mathbb{Z}_{0}} \prod_{i=1}^{p}\left(1+\left|k_{i}\right|^{2}\right)^{\alpha}\left(k_{i}^{2}\right)^{\kappa}\left(\left|X_{0}\left(e_{k_{i}}\right)\right|^{2}+1\right) \\
& \lesssim(t-s)^{\kappa p}\left[\sum_{k \in \mathbb{Z}_{0}}\left(1+|k|^{2}\right)^{\alpha}\left(k^{2}\right)^{\kappa}\left(\left|X_{0}\left(e_{k}\right)\right|^{2}+1\right)\right]^{p} \\
& \lesssim(t-s)^{\kappa p}\left(\left\|X_{0}\right\|_{H^{\alpha+\kappa}(\mathbb{T})}^{2 p}+\left[\sum_{k \in \mathbb{Z}_{0}}\left(1+|k|^{2}\right)^{\alpha}\left(k^{2}\right)^{\kappa}\right]^{p}\right) .
\end{aligned}
$$

If $\alpha<-1 / 2-\kappa$, the sum on the right hand side is finite and we obtain an estimation for the modulus of continuity of $t \mapsto X_{t}$ in $L^{2 p}\left(\Omega ; H^{\alpha}\right)$ :

$$
\mathbb{E}\left\|X_{t}-X_{s}\right\|_{H^{\alpha}}^{2 p} \lesssim(t-s)^{\kappa p}\left[1+\left\|X_{0}\right\|_{H^{\alpha+\kappa}}^{2 p}\right] .
$$

Now Kolmogorov's continuity criterion allows us to conclude that almost surely $X \in C H^{\alpha}$ whenever $X_{0} \in H^{\alpha+\kappa}$.

Now note that the regularity of the Ornstein-Uhlenbeck process does not allow us to form the quantity $X_{t}^{2}$ point-wise in time since by Fourier inversion $X_{t}=\sum_{k} X_{t}\left(e_{k}\right) e_{k}^{*}$, and therefore we should have

$$
X_{t}^{2}\left(e_{k}\right)=(2 \pi)^{-1 / 2} \sum_{\ell+m=k} X_{t}\left(e_{\ell}\right) X_{t}\left(e_{m}\right) .
$$

Of course, at the moment this expression is purely formal since we cannot guarantee that the infinite sum converges. A reasonable thing to try is to approximate the square by regularizing the distribution, taking the square, and then trying to remove the regularization. Let $\Pi_{N}$ be the projector of a distribution onto a finite number of Fourier modes:

$$
\left(\Pi_{N} \rho\right)(x)=\sum_{|k| \leqslant N} \rho\left(e_{k}\right) e_{k}^{*}(x) .
$$

Then $\Pi_{N} X_{t}(x)$ is a smooth function of $x$ and we can consider $\left(\Pi_{N} X_{t}\right)^{2}$ which satisfies

$$
\left(\Pi_{N} X_{t}\right)^{2}\left(e_{k}\right)=(2 \pi)^{-1 / 2} \sum_{\ell+m=k} \mathbb{I}_{|\ell| \leqslant N,|m| \leqslant N} X_{t}\left(e_{\ell}\right) X_{t}\left(e_{m}\right) .
$$


We would then like to take the limit $N \rightarrow+\infty$. For convenience, we will perform the computations below in the limit $N=+\infty$, but one has to come back to the case of finite $N$ in order to make it rigorous.

Then

$$
\begin{aligned}
\mathbb{E}\left[X_{t}^{2}\left(e_{k}\right)\right]= & (2 \pi)^{-1 / 2} \delta_{k=0} \sum_{m \in \mathbb{Z}_{0}} \mathbb{E}\left[X_{t}\left(e_{-m}\right) X_{t}\left(e_{m}\right)\right] \\
= & (2 \pi)^{-1 / 2} \delta_{k=0} \sum_{m \in \mathbb{Z}_{0}} e^{-2 m^{2} t}\left|X_{0}\left(e_{m}\right)\right|^{2} \\
& +(2 \pi)^{-1 / 2} \delta_{k=0} \sum_{m \in \mathbb{Z}_{0}} m^{2} \int_{0}^{t} e^{-2 m^{2}(t-s)} \mathrm{d} s
\end{aligned}
$$

and

$$
\sum_{m \in \mathbb{Z}_{0}} m^{2} \int_{0}^{t} e^{-2 m^{2}(t-s)} \mathrm{d} s=\frac{1}{2} \sum_{m \in \mathbb{Z}_{0}}\left(1-e^{-2 m^{2} t}\right)=+\infty .
$$

This is not really a problem since in Burgers' equation only components of $u_{t}^{2}\left(e_{k}\right)$ with $k \neq 0$ appear (due to the presence of the derivative). However, $X_{t}^{2}\left(e_{k}\right)$ is not even a well-defined random variable. For the remainder of this subsection let us assume that $X_{0}=0$, which will slightly simplify the computation. If $k \neq 0$, we have

$$
\begin{aligned}
\mathbb{E}\left[\left|X_{t}^{2}\left(e_{k}\right)\right|^{2}\right] & =\mathbb{E}\left[X_{t}^{2}\left(e_{k}\right) X_{t}^{2}\left(e_{-k}\right)\right] \\
& =(2 \pi)^{-1} \sum_{\ell+m=k} \sum_{\ell^{\prime}+m^{\prime}=-k} \mathbb{E}\left[X_{t}\left(e_{\ell}\right) X_{t}\left(e_{m}\right) X_{t}\left(e_{\ell^{\prime}}\right) X_{t}\left(e_{m^{\prime}}\right)\right] .
\end{aligned}
$$

By Wick's theorem (see [22], Theorem 1.28), the expectation can be computed in terms of the covariances of all possible pairings of the four Gaussian random variables (3 possible combinations):

$$
\begin{aligned}
\mathbb{E}\left[X_{t}\left(e_{\ell}\right) X_{t}\left(e_{m}\right) X_{t}\left(e_{\ell^{\prime}}\right) X_{t}\left(e_{m^{\prime}}\right)\right]= & \mathbb{E}\left[X_{t}\left(e_{\ell}\right) X_{t}\left(e_{m}\right)\right] \mathbb{E}\left[X_{t}\left(e_{\ell^{\prime}}\right) X_{t}\left(e_{m^{\prime}}\right)\right] \\
& +\mathbb{E}\left[X_{t}\left(e_{\ell}\right) X_{t}\left(e_{\ell^{\prime}}\right)\right] \mathbb{E}\left[X_{t}\left(e_{m}\right) X_{t}\left(e_{m^{\prime}}\right)\right] \\
& +\mathbb{E}\left[X_{t}\left(e_{\ell}\right) X_{t}\left(e_{m^{\prime}}\right)\right] \mathbb{E}\left[X_{t}\left(e_{m}\right) X_{t}\left(e_{\ell^{\prime}}\right)\right] .
\end{aligned}
$$

Since $k \neq 0$, we have $\ell+m \neq 0$ and $\ell^{\prime}+m^{\prime} \neq 0$ which allows us to neglect the first term since it is zero. By symmetry of the summations, the two other terms give the same contribution and we remain with

$$
\begin{aligned}
\mathbb{E}\left[\left|X_{t}^{2}\left(e_{k}\right)\right|^{2}\right] & =\frac{1}{\pi} \sum_{\ell+m=k} \sum_{\ell^{\prime}+m^{\prime}=-k} \mathbb{E}\left[X_{t}\left(e_{\ell}\right) X_{t}\left(e_{\ell^{\prime}}\right)\right] \mathbb{E}\left[X_{t}\left(e_{m}\right) X_{t}\left(e_{m^{\prime}}\right)\right] \\
& =\frac{1}{\pi} \sum_{\ell+m=k} \mathbb{E}\left[X_{t}\left(e_{\ell}\right) X_{t}\left(e_{-\ell}\right)\right] \mathbb{E}\left[X_{t}\left(e_{m}\right) X_{t}\left(e_{-m}\right)\right] \\
& =\frac{1}{4 \pi} \sum_{\ell+m=k}\left(1-e^{-2 \ell^{2} t}\right)\left(1-e^{-2 m^{2} t}\right)=+\infty
\end{aligned}
$$


This shows that even when tested against smooth test functions, $X_{t}^{2}$ is not in $L^{2}(\Omega)$. This indicates that there are problems with $X_{t}^{2}$ and indeed one can show that $X_{t}^{2}\left(e_{k}\right)$ does not make sense as a random variable.

To understand this better, observe that the Ornstein-Uhlenbeck process can be decomposed as

$$
X_{t}\left(e_{k}\right)=i k \int_{-\infty}^{t} e^{-k^{2}(t-s)} \mathrm{d} \beta_{s}(k)-i k e^{-k^{2} t} \int_{-\infty}^{0} e^{k^{2} s} \mathrm{~d} \beta_{s}(k),
$$

where we extended the Brownian motions $\left(\beta_{s}(k)\right)_{s \geqslant 0}$ to two sided complex Brownian motions by considering independent copies. The interest in this decomposition is in the fact that it is not difficult to show that the second term gives rise to a smooth function if $t>0$, so all the irregularity of $X_{t}$ is described by the first term which we call $Y_{t}\left(e_{k}\right)$ and which is stationary in time. Note that $Y_{t}\left(e_{k}\right) \sim \mathcal{N}_{\mathbb{C}}(0,1 / 2)$ for all $k \in \mathbb{Z}_{0}$ and $t \in \mathbb{R}$, where we write

$$
U \sim \mathcal{N}_{\mathbb{C}}\left(0, \sigma^{2}\right)
$$

if $U=V+i W$, where $V$ and $W$ are independent random variables with distribution $\mathcal{N}\left(0, \sigma^{2} / 2\right)$. The random distribution $Y_{t}$ then satisfies $Y_{t}(\varphi) \sim \mathcal{N}\left(0,\|\varphi\|_{L^{2}(\mathbb{T})}^{2} / 2\right)$, and moreover it is $(1 / \sqrt{2}$ times $)$ the white noise on $\mathbb{T}$. It is also possible to deduce that the white noise on $\mathbb{T}$ is indeed the invariant measure of the Ornstein-Uhlenbeck process, that it is the only one, and that it is approached quite fast [23].

So we should expect that, at fixed time, the regularity of the OrnsteinUhlenbeck process is like that of the space white noise and this is a way of understanding our difficulties in defining $X_{t}^{2}$ since this will be, modulo smooth terms, the square of the space white noise.

A different matter is to make sense of the time-integral of $\partial_{x} X_{t}^{2}$. Let us give it a name and call it $J_{t}(\varphi)=\int_{0}^{t} \partial_{x} X_{s}^{2}(\varphi) \mathrm{d} s$. For $J_{t}\left(e_{k}\right)$, the computation of its variance gives a quite different result.

Lemma 2. Almost surely, $J \in C^{1 / 2-} H^{-1 / 2-}$.

Proof. Proceeding as in (2.2), we have now

$$
\mathbb{E}\left[\left|J_{t}\left(e_{k}\right)\right|^{2}\right]=\frac{1}{\pi} k^{2} \int_{0}^{t} \int_{0}^{t} \sum_{\ell+m=k} \mathbb{E}\left[X_{s}\left(e_{\ell}\right) X_{s^{\prime}}\left(e_{-\ell}\right)\right] \mathbb{E}\left[X_{s}\left(e_{m}\right) X_{s^{\prime}}\left(e_{-m}\right)\right] \mathrm{d} s \mathrm{~d} s^{\prime} .
$$

If $s>s^{\prime}$, we have

$$
\mathbb{E}\left[X_{s}\left(e_{\ell}\right) X_{s^{\prime}}\left(e_{-\ell}\right)\right]=\frac{1}{2} e^{-\ell^{2}\left(s-s^{\prime}\right)}\left(1-e^{-2 \ell^{2} s^{\prime}}\right),
$$


and therefore

$$
\begin{aligned}
\mathbb{E}\left[\left|J_{t}\left(e_{k}\right)\right|^{2}\right] & =\frac{k^{2}}{4 \pi} \int_{0}^{t} \int_{0}^{t} \sum_{\ell+m=k} e^{-\left(\ell^{2}+m^{2}\right)\left|s-s^{\prime}\right|}\left(1-e^{-2 \ell^{2}\left(s^{\prime} \wedge s\right)}\right)\left(1-e^{-2 m^{2}\left(s^{\prime} \wedge s\right)}\right) \mathrm{d} s \mathrm{~d} s^{\prime} \\
& \leqslant \frac{k^{2}}{4 \pi} \int_{0}^{t} \int_{0}^{t} \sum_{\ell+m=k} e^{-\left(\ell^{2}+m^{2}\right)\left|s-s^{\prime}\right|} \mathrm{d} s \mathrm{~d} s^{\prime} \\
& \leqslant \frac{1}{2 \pi} k^{2} t \sum_{\ell+m=k} \int_{0}^{\infty} e^{-\left(\ell^{2}+m^{2}\right) r} \mathrm{~d} r \\
& =\frac{1}{2 \pi} k^{2} t \sum_{\ell+m=k} \frac{1}{\ell^{2}+m^{2}} .
\end{aligned}
$$

Now for $k \neq 0$

$$
\sum_{\ell+m=k} \frac{1}{\ell^{2}+m^{2}} \lesssim \int_{\mathbb{R}} \frac{\mathrm{d} x}{x^{2}+(k-x)^{2}} \lesssim \frac{1}{|k|}
$$

So finally $\mathbb{E}\left[\left|J_{t}\left(e_{k}\right)\right|^{2}\right] \lesssim|k| t$. From which is easy to conclude that at fixed $t$ the random field $J_{t}$ belongs almost surely to $H^{-1 / 2-}$. Redoing a similar computation in the case $J_{t}\left(e_{k}\right)-J_{s}\left(e_{k}\right)$, we obtain $\mathbb{E}\left[\left|J_{t}\left(e_{k}\right)-J_{s}\left(e_{k}\right)\right|^{2}\right] \lesssim$ $|k| \times|t-s|$. To go from this estimate to a path-wise regularity result of the distribution $\left(J_{t}\right)_{t}$, following the line of reasoning of Lemma 1, we need to estimate the $p$-th moment of $J_{t}\left(e_{k}\right)-J_{s}\left(e_{k}\right)$. We already used in the proof of Lemma 1 that all moments of a Gaussian random variable are comparable. By Gaussian hypercontractivity (see Theorem 3.50 of [22]) this also holds for polynomials of Gaussian random variables, so that

$$
\mathbb{E}\left[\left|J_{t}\left(e_{k}\right)-J_{s}\left(e_{k}\right)\right|^{2 p}\right] \lesssim_{p}\left(\mathbb{E}\left[\left|J_{t}\left(e_{k}\right)-J_{s}\left(e_{k}\right)\right|^{2}\right]\right)^{p}
$$

From here we easily derive that almost surely $J \in C^{1 / 2-} H^{-1 / 2-}$ which is the space of $1 / 2$-Hölder continuous functions with values in $H^{-1 / 2-}$.

This shows that $\partial_{x} X_{t}^{2}$ exists as a space-time distribution but not as a continuous function of time with values in distributions in space. The key point in the proof of Lemma 2 is the fact that the correlation $\mathbb{E}\left[X_{s}\left(e_{\ell}\right) X_{s^{\prime}}\left(e_{-\ell}\right)\right]$ of the Ornstein-Uhlenbeck process decays quite rapidly in time.

The construction of the process $J$ does not solve our problem of constructing $\int_{0}^{t} \partial_{x} u_{s}^{2} \mathrm{~d} s$ since we need similar properties for the full solution $u$ of the non-linear dynamics (or for some approximations thereof), and all we have done so far relies on explicit computations and the specific Gaussian features of the Ornstein-Uhlenbeck process. But at least this give us a hint that indeed there could exist a way of making sense of the term $\partial_{x} u(t, x)^{2}$, even if only as a space-time distribution, and that in doing so we should exploit some decorrelation properties of the dynamics. 
So when dealing with the full solution $u$, we need a replacement for the Gaussian computations based on the explicit distribution of $X$ that we used above. This will be provided, in the current setting, by stochastic calculus along the time direction. Indeed, note that for each $\varphi \in \mathscr{S}$ the process $\left(X_{t}(\varphi)\right)_{t \geqslant 0}$ is a semimartingale in the filtration $\left(\mathcal{F}_{t}\right)_{t \geqslant 0}$.

Before proceeding with these computations, we need to develop some tools to describe the Itô formula for functions of the Ornstein-Uhlenbeck process. This will also serve us as an opportunity to set up some analysis on Gaussian spaces.

\subsection{Gaussian computations}

For cylindrical functions $F: \mathscr{S}^{\prime} \rightarrow \mathbb{R}$ of the form $F(\rho)=$ $f\left(\rho\left(\varphi_{1}\right), \ldots, \rho\left(\varphi_{n}\right)\right)$ with $\varphi_{1}, \ldots, \varphi_{n} \in \mathscr{S}$ and $f: \mathbb{R}^{n} \rightarrow \mathbb{R}$ at least $C_{b}^{2}$, we have by Itô's formula

$$
\mathrm{d}_{t} F\left(X_{t}\right)=\sum_{i=1}^{n} F_{i}\left(X_{t}\right) \mathrm{d} X_{t}\left(\varphi_{i}\right)+\frac{1}{2} \sum_{i, j=1}^{n} F_{i, j}\left(X_{t}\right) \mathrm{d}\left\langle X\left(\varphi_{i}\right), X\left(\varphi_{j}\right)\right\rangle_{t}
$$

where \langle\rangle$_{t}$ denotes the quadratic covariation of two continuous semimartingales and where $F_{i}(\rho)=\partial_{i} f\left(\rho\left(\varphi_{1}\right), \ldots, \rho\left(\varphi_{n}\right)\right)$ and $F_{i, j}(\rho)=$ $\partial_{i, j}^{2} f\left(\rho\left(\varphi_{1}\right), \ldots, \rho\left(\varphi_{n}\right)\right)$, with $\partial_{i}$ denoting the derivative with respect to the $i$-th argument. Now recall that $\mathrm{d} X_{t}\left(\varphi_{i}\right)=X_{t}\left(\Delta \varphi_{i}\right) \mathrm{d} t+\mathrm{d} M_{t}\left(\varphi_{i}\right)$ is a continuous semimartingale, and therefore

$$
\mathrm{d}\left\langle X\left(\varphi_{i}\right), X\left(\varphi_{j}\right)\right\rangle_{t}=\mathrm{d}\left\langle M\left(\varphi_{i}\right), M\left(\varphi_{j}\right)\right\rangle_{t}=\left\langle\partial_{x} \varphi_{i}, \partial_{x} \varphi_{j}\right\rangle_{L^{2}(\mathbb{T})} \mathrm{d} t
$$

and then

$$
\mathrm{d}_{t} F\left(X_{t}\right)=\sum_{i=1}^{n} F_{i}\left(X_{t}\right) \mathrm{d} M_{t}\left(\varphi_{i}\right)+L_{0} F\left(X_{t}\right) \mathrm{d} t
$$

where $L_{0}$ is the second-order differential operator defined on cylindrical functions $F$ as

$$
L_{0} F(\rho)=\sum_{i=1}^{n} F_{i}(\rho) \rho\left(\Delta \varphi_{i}\right)+\sum_{i, j=1}^{n} \frac{1}{2} F_{i, j}(\rho)\left\langle\partial_{x} \varphi_{i}, \partial_{x} \varphi_{j}\right\rangle_{L^{2}(\mathbb{T})}
$$

Another way to describe the generator $L_{0}$ is to give its value on the functions $\rho \mapsto \exp (\rho(\psi))$ for $\psi \in \mathscr{S}$, which is

$$
L_{0} e^{\rho(\psi)}=e^{\rho(\psi)}\left(\rho(\Delta \psi)-\frac{1}{2}\langle\psi, \Delta \psi\rangle_{L^{2}(\mathbb{T})}\right) .
$$


If $F, G$ are two cylindrical functions (which we can take of the form $F(\rho)=f\left(\rho\left(\varphi_{1}\right), \ldots, \rho\left(\varphi_{n}\right)\right)$ and $G(\rho)=g\left(\rho\left(\varphi_{1}\right), \ldots, \rho\left(\varphi_{n}\right)\right)$ for the same $\left.\varphi_{1}, \ldots, \varphi_{n} \in \mathscr{S}\right)$, we can check that

$$
L_{0}(F G)=\left(L_{0} F\right) G+F\left(L_{0} G\right)+\mathcal{E}(F, G),
$$

where the quadratic form $\mathcal{E}$ is given by

$$
\mathcal{E}(F, G)(\rho)=\sum_{i, j} F_{i}(\rho) G_{j}(\rho)\left\langle\partial_{x} \varphi_{i}, \partial_{x} \varphi_{j}\right\rangle_{L^{2}(\mathbb{T})} .
$$

In particular, the quadratic variation of the martingale obtained in the Itô formula for $F$ is given by

$$
\mathrm{d}\left\langle\int_{0} \sum_{i=1}^{n} F_{i}\left(X_{s}\right) \mathrm{d} M_{s}\left(\varphi_{i}\right)\right\rangle_{t}=\mathcal{E}(F, F)\left(X_{t}\right) \mathrm{d} t .
$$

Lemma 3. (Gaussian integration by parts) Let $\left(Z_{i}\right)_{i=1, \ldots, M}$ be an $M$-dimensional Gaussian vector with zero mean and covariance $\left(C_{i, j}\right)_{i, j=1, \ldots, M}$. Then for all $g \in C_{b}^{1}\left(\mathbb{R}^{M}\right)$ we have

$$
\mathbb{E}\left[Z_{k} g(Z)\right]=\sum_{\ell} C_{k, \ell} \mathbb{E}\left[\frac{\partial g(Z)}{\partial Z_{\ell}}\right] .
$$

Proof. Use that $\mathbb{E}\left[e^{i\langle Z, \lambda\rangle}\right]=e^{-\langle\lambda, C \lambda\rangle / 2}$ and moreover that

$$
\begin{aligned}
\mathbb{E}\left[Z_{k} e^{i\langle Z, \lambda\rangle}\right] & =(-i) \frac{\partial}{\partial \lambda_{k}} \mathbb{E}\left[e^{i\langle Z, \lambda\rangle}\right]=(-i) \frac{\partial}{\partial \lambda_{k}} e^{-\langle\lambda, C \lambda\rangle / 2}=i(C \lambda)_{k} e^{-\langle\lambda, C \lambda\rangle / 2} \\
& =i \sum_{\ell} C_{k, \ell} \lambda_{\ell} \mathbb{E}\left[e^{i\langle Z, \lambda\rangle}\right]=\sum_{\ell} C_{k, \ell} \mathbb{E}\left[\frac{\partial}{\partial Z_{\ell}} e^{i\langle Z, \lambda\rangle}\right] .
\end{aligned}
$$

The relation is true for trigonometric functions and taking Fourier transforms we see that it holds for all $g \in \mathscr{S}$. Is then a matter of taking limits to show that we can extend it to any $g \in C_{b}^{1}\left(\mathbb{R}^{M}\right)$.

As a first application of this formula let us show that $\mathbb{E}\left[L_{0} F(\eta)\right]=0$ for every cylindrical function, where $\eta$ is a space white noise with mean zero, i.e. $\eta(\varphi) \sim \mathcal{N}\left(0,\|\varphi\|_{L^{2}(\mathbb{T})}^{2} / 2\right)$ for all $\varphi \in L_{0}^{2}(\mathbb{T})$, and $\eta(1)=0$. Here we write $L_{0}^{2}(\mathbb{T})$ for the subspace of all $\varphi \in L^{2}(\mathbb{T})$ with $\int_{\mathbb{T}} \varphi \mathrm{d} x=0$. Indeed, note that by polarization $\mathbb{E}\left[\eta\left(\varphi_{i}\right) \eta\left(\Delta \varphi_{j}\right)\right]=\frac{1}{2}\left\langle\varphi_{i}, \Delta \varphi_{j}\right\rangle_{L^{2}(\mathbb{T})}$, leading to

$$
\begin{aligned}
\mathbb{E} \sum_{i, j=1}^{n} \frac{1}{2} F_{i, j}(\eta)\left\langle\partial_{x} \varphi_{i}, \partial_{x} \varphi_{j}\right\rangle_{L^{2}(\mathbb{T})} & =-\mathbb{E} \sum_{i, j=1}^{n} \frac{1}{2} F_{i, j}(\eta)\left\langle\varphi_{i}, \Delta \varphi_{j}\right\rangle_{L^{2}(\mathbb{T})} \\
& =-\frac{1}{2} \sum_{i, j=1}^{n}\left\langle\varphi_{i}, \Delta \varphi_{j}\right\rangle_{L^{2}(\mathbb{T})} \mathbb{E} \frac{\partial}{\partial \eta\left(\varphi_{i}\right)} F_{j}(\eta) \\
& =-\sum_{j=1}^{n} \mathbb{E}\left[\eta\left(\Delta \varphi_{j}\right) F_{j}(\eta)\right],
\end{aligned}
$$


so that $\mathbb{E}\left[L_{0} F(\eta)\right]=0$ (here we interpreted $\partial_{j} f$ as a function of $n+1$ variables, with trivial dependence on the $(n+1)$-th one). In combination with Itô's formula, this indicates that the white noise law should indeed be a stationary distribution for $X$ (convince yourself of it!). From now on we fix the initial distribution $X_{0} \sim \eta$, which means that $X_{t} \sim \eta$ for all $t \geqslant 0$.

As another application of the Gaussian integration by parts formula, we get

$$
\begin{aligned}
\frac{1}{2} \mathbb{E}[\mathcal{E}(F, G)(\eta)]=- & \frac{1}{2} \sum_{i, j} \mathbb{E}\left[F_{i}(\eta) G_{j}(\eta)\right]\left\langle\varphi_{i}, \Delta \varphi_{j}\right\rangle_{L^{2}(\mathbb{T})} . \\
=- & \frac{1}{2} \sum_{i, j} \mathbb{E}\left[\left(F(\eta) G_{j}(\eta)\right)_{i}\right]\left\langle\varphi_{i}, \Delta \varphi_{j}\right\rangle_{L^{2}(\mathbb{T})} \\
& +\frac{1}{2} \sum_{i, j} \mathbb{E}\left[F(\eta) G_{i j}(\eta)\right]\left\langle\varphi_{i}, \Delta \varphi_{j}\right\rangle_{L^{2}(\mathbb{T})} \\
=- & \sum_{j} \mathbb{E}\left[F(\eta) G_{j}(\eta) \eta\left(\Delta \varphi_{j}\right)\right] \\
& +\frac{1}{2} \sum_{i, j} \mathbb{E}\left[F(\eta) G_{i j}(\eta)\right]\left\langle\varphi_{i}, \Delta \varphi_{j}\right\rangle_{L^{2}(\mathbb{T})} \\
=- & \mathbb{E}\left[\left(F L_{0} G\right)(\eta)\right] .
\end{aligned}
$$

Combining this with (2.4) and with $\mathbb{E}\left[L_{0}(F G)(\eta)\right]=0$, we obtain $\mathbb{E}\left[\left(F L_{0} G\right)(\eta)\right]=\mathbb{E}\left[\left(G L_{0} F\right)(\eta)\right]$. That is, $L_{0}$ is a symmetric operator with respect to the law of $\eta$.

Consider now the operator D, defined on cylindrical functions $F$ by

$$
\mathrm{D} F(\rho)=\sum_{i} F_{i}(\rho) \varphi_{i}
$$

so that $\mathrm{D} F$ takes values in $\mathscr{S}^{\prime}$, the continuous linear functionals on $\mathscr{S}$.

Exercise 5. Show that $\mathrm{D}$ is independent of the specific representation of $F$, that is if

$$
F(\rho)=f\left(\rho\left(\varphi_{1}\right), \ldots, \rho\left(\varphi_{n}\right)\right)=g\left(\rho\left(\psi_{1}\right), \ldots, \rho\left(\psi_{m}\right)\right)
$$

for all $\rho \in \mathscr{S}^{\prime}$, then

$$
\sum_{i} \partial_{i} f\left(\rho\left(\varphi_{1}\right), \ldots, \rho\left(\varphi_{n}\right)\right) \varphi_{i}=\sum_{j} \partial_{j} g\left(\rho\left(\psi_{1}\right), \ldots, \rho\left(\psi_{m}\right)\right) \psi_{m} .
$$

Hint: One possible strategy is to show that for all $\theta \in \mathscr{S}$,

$$
\langle\mathrm{D} F(\rho), \theta\rangle=\left.\frac{\mathrm{d}}{\mathrm{d} \varepsilon} F(\rho+\varepsilon \theta)\right|_{\varepsilon=0} .
$$


By Gaussian integration by parts we get

$$
\begin{aligned}
\mathbb{E}[F(\eta)\langle\psi, \mathrm{D} G(\eta)\rangle]+\mathbb{E}[G(\eta)\langle\psi, \mathrm{D} F(\eta)\rangle] & =\sum_{i} \mathbb{E}\left[(F G)_{i}(\eta)\left\langle\psi, \varphi_{i}\right\rangle\right] \\
& =2 \mathbb{E}[\eta(\psi)(F G)(\eta)]
\end{aligned}
$$

and therefore

$$
\mathbb{E}[F(\eta)\langle\psi, \mathrm{D} G(\eta)\rangle]=\mathbb{E}[G(\eta)\langle\psi,-\mathrm{D} F(\eta)+2 \rho F(\eta)\rangle] .
$$

So if we consider the space $L^{2}(\operatorname{law}(\eta))$ with inner product $\mathbb{E}[F(\eta) G(\eta)]$, then the adjoint of $\mathrm{D}$ is given by $\mathrm{D}^{*} F(\rho)=-\mathrm{D} F(\rho)+2 \rho F(\rho)$. Let $\mathrm{D}_{\psi} F(\rho)=\langle\psi, \mathrm{D} F(\rho)\rangle$ and similarly for $\mathrm{D}_{\psi}^{*} F(\rho)=-\mathrm{D}_{\psi} F(\rho)+2 \rho(\psi) F(\rho)$.

Exercise 6. Let $\left(e_{n}\right)_{n \geqslant 1}$ be an orthonormal basis of $L^{2}(\mathbb{T})$. Show that

$$
L_{0}=\frac{1}{2} \sum_{n} \mathrm{D}_{e_{n}}^{*} \mathrm{D}_{\Delta e_{n}} .
$$

Recall that the commutator between two operators $A$ and $B$ is defined as $[A, B]:=A B-B A$. In our case we have

$$
\left[\mathrm{D}_{\theta}, \mathrm{D}_{\psi}^{*}\right] F(\rho)=\left(\mathrm{D}_{\theta} \mathrm{D}_{\psi}^{*}-\mathrm{D}_{\psi}^{*} \mathrm{D}_{\theta}\right) F(\rho)=2\langle\psi, \theta\rangle_{L^{2}(\mathbb{T})} F(\rho),
$$

whereas $\left[\mathrm{D}_{\theta}^{*}, \mathrm{D}_{\psi}^{*}\right]=0$. Therefore,

$$
\begin{aligned}
{\left[L_{0}, \mathrm{D}_{\psi}^{*}\right] } & =\frac{1}{2} \sum_{n}\left[\mathrm{D}_{e_{n}}^{*} \mathrm{D}_{\Delta e_{n}}, \mathrm{D}_{\psi}^{*}\right] \\
& =\frac{1}{2} \sum_{n} \mathrm{D}_{e_{n}}^{*}\left[\mathrm{D}_{\Delta e_{n}}, \mathrm{D}_{\psi}^{*}\right]+\frac{1}{2} \sum_{n}\left[\mathrm{D}_{e_{n}}^{*}, \mathrm{D}_{\psi}^{*}\right] \mathrm{D}_{\Delta e_{n}} \\
& =\sum_{n} \mathrm{D}_{e_{n}}^{*}\left\langle\psi, \Delta e_{n}\right\rangle_{L^{2}(\mathbb{T})}=\mathrm{D}_{\Delta \psi}^{*}
\end{aligned}
$$

So if $\psi$ is an eigenvector of $\Delta$ with eigenvalue $\lambda$, then $\left[L_{0}, D_{\psi}^{*}\right]=\lambda \mathrm{D}_{\psi}^{*}$. Let now $\left(\psi_{n}\right)_{n \in \mathbb{N}}$ be an orthonormal eigenbasis for $\Delta$ with eigenvalues $\Delta \psi_{n}=\lambda_{n} \psi_{n}$ and consider the functions

$$
H\left(\psi_{i_{1}}, \ldots, \psi_{i_{n}}\right): \mathscr{S}^{\prime} \rightarrow \mathbb{R}, \quad H\left(\psi_{i_{1}}, \ldots, \psi_{i_{n}}\right)(\rho)=\left(\mathrm{D}_{\psi_{i_{1}}}^{*} \cdots \mathrm{D}_{{\psi_{i_{n}}}_{1}}^{*} 1\right)(\rho)
$$

Then

$$
\begin{aligned}
L_{0} H\left(\psi_{i_{1}}, \ldots, \psi_{i_{n}}\right) & =L_{0} \mathrm{D}_{\psi_{i_{1}}}^{*} \cdots \mathrm{D}_{\psi_{i_{n}}}^{*} 1 \\
& =\mathrm{D}_{\psi_{i_{1}}}^{*} L_{0} \mathrm{D}_{\psi_{i_{2}}}^{*} \cdots \mathrm{D}_{\psi_{i_{n}}}^{*} 1+\lambda_{i_{1}} \mathrm{D}_{\psi_{i_{1}}}^{*} \cdots \mathrm{D}_{\psi_{i_{n}}}^{*} 1 \\
& =\cdots=\left(\lambda_{i_{1}}+\cdots+\lambda_{i_{n}}\right) H\left(\psi_{i_{1}}, \ldots, \psi_{i_{n}}\right),
\end{aligned}
$$


where we used that $L_{0} 1=0$. So these functions are eigenfunctions for $L_{0}$ and the eigenvalues are all the possible combinations of $\lambda_{i_{1}}+\cdots+\lambda_{i_{n}}$ for $i_{1}, \ldots, i_{n} \in \mathbb{N}$. We have immediately that for different $n$ these functions are orthogonal in $L^{2}(\operatorname{law}(\eta))$. They are actually orthogonal as soon as the indices $i$ differ since in that case there is an index $j$ which is in one but not in the other and using the fact that $\mathrm{D}_{\psi_{j}}^{*}$ is adjoint to $D_{\psi_{j}}$ and that $D_{\psi_{j}} G=0$ if $G$ does not depend on $\psi_{j}$ we get the orthogonality. The functions $H\left(\psi_{i_{1}}, \ldots, \psi_{i_{n}}\right)$ are polynomials and they are called Wick polynomials.

Lemma 4. For all $\psi \in \mathscr{S}$, almost surely

$$
\left(e^{\mathrm{D}_{\psi}^{*}} 1\right)(\eta)=e^{2 \eta(\psi)-\|\psi\|^{2}} .
$$

Proof. If $F$ is a cylindrical function of the form $F(\rho)=$ $f\left(\rho\left(\varphi_{1}\right), \ldots, \rho\left(\varphi_{m}\right)\right)$ with $f \in \mathscr{S}\left(\mathbb{R}^{m}\right)$, then

$$
\mathbb{E}\left[F(\eta)\left(e^{\mathrm{D}_{\psi}^{*}} 1\right)(\eta)\right]=\mathbb{E}\left[e^{\mathrm{D}_{\psi}} F(\eta)\right]=\mathbb{E}[F(\eta+\psi)]=\mathbb{E}\left[F(\eta) e^{2 \eta(\psi)-\|\psi\|^{2}}\right],
$$

where the second step follows from the fact that if we note $\Psi_{t}(\eta)=$ $F(\eta+t \psi)$ (note that every $\psi \in \mathscr{S}$ can be interpreted as an element of $\mathscr{S}^{\prime}$ ) we have $\partial_{t} \Psi_{t}(\eta)=\mathrm{D}_{\psi} \Psi_{t}(\eta)$ and $\Psi_{0}(\eta)=F(\eta)$ so that $\Psi_{t}(\eta)=\left(e^{t \mathrm{D}_{\psi}} F\right)(\eta)$ for all $t \geqslant 0$ and in particular for $t=1$. The last step is simply a Gaussian change of variables. Indeed if we take $\varphi_{1}=\psi$ and $\varphi_{k} \perp \psi$ for $k \geqslant 2$ we have

$$
\mathbb{E}[F(\eta+\psi)]=\mathbb{E}\left[f\left(\eta(\psi)+\langle\psi, \psi\rangle, \eta\left(\varphi_{2}\right), \ldots, \eta\left(\varphi_{m}\right)\right)\right]
$$

since $(\eta+\psi)\left(\varphi_{k}\right)=\eta\left(\varphi_{k}\right)$ for $k \geqslant 2$. Now observe that $\eta(\psi)$ is independent of $\left(\eta\left(\varphi_{2}\right), \ldots, \eta\left(\varphi_{m}\right)\right)$ so that

$$
\begin{aligned}
& \mathbb{E}\left[f\left(\eta(\psi)+\langle\psi, \psi\rangle, \eta\left(\varphi_{2}\right), \ldots, \eta\left(\varphi_{m}\right)\right)\right] \\
= & \int_{\mathbb{R}} \frac{e^{-z^{2} /\|\psi\|^{2}}}{\sqrt{\pi\|\psi\|^{2}}} \mathbb{E}\left[f\left(z+\langle\psi, \psi\rangle, \eta\left(\varphi_{2}\right), \ldots, \eta\left(\varphi_{m}\right)\right)\right] \\
= & \int_{\mathbb{R}} \frac{e^{-z^{2} /\|\psi\|^{2}}}{\sqrt{\pi\|\psi\|^{2}}} e^{2 z-\|\psi\|^{2}} \mathbb{E}\left[f\left(z, \eta\left(\varphi_{2}\right), \ldots, \eta\left(\varphi_{m}\right)\right)\right] \\
= & \mathbb{E}\left[F(\eta) e^{2 \eta(\psi)-\|\psi\|^{2}}\right] .
\end{aligned}
$$

To conclude the proof, it suffices to note that $\mathbb{E}\left[F(\eta)\left(e^{\mathrm{D}_{\psi}^{*}} 1\right)(\eta)\right]=$ $\mathbb{E}\left[F(\eta) e^{2 \eta(\psi)-\|\psi\|^{2}}\right]$ for all cylindrical functions $F$ implies that $\left(e^{\mathrm{D}_{\psi}^{*}} 1\right)(\eta)=$ $e^{2 \eta(\psi)-\|\psi\|^{2}}$.

Theorem 1. The Wick polynomials $\left\{H\left(\psi_{i_{1}}, \ldots, \psi_{i_{n}}\right)(\eta): n \geqslant\right.$ $\left.0, i_{1}, \ldots, i_{n} \in \mathbb{N}\right\}$ form an orthogonal basis of $L^{2}(\operatorname{law}(\eta))$. 
Proof. Taking $\psi=\sum_{i} \sigma_{i} \psi_{i}$ in Lemma 4, we get

$$
\begin{aligned}
e^{2 \sum_{i} \sigma_{i} \eta\left(\psi_{i}\right)-\sum_{i} \sigma_{i}^{2}\left\|\psi_{i}\right\|^{2}} & =\left(e^{\mathrm{D}_{\psi}^{*}} 1\right)(\eta)=\sum_{n \geqslant 0} \frac{\left(\left(\mathrm{D}_{\psi}^{*}\right)^{n} 1\right)(\eta)}{n !} \\
& =\sum_{n \geqslant 0} \sum_{i_{1}, \ldots, i_{n}} \frac{\sigma_{i_{1}} \cdots \sigma_{i_{n}}}{n !} H(\underbrace{\psi_{i_{1}}, \ldots, \psi_{i_{n}}}_{n \text { times }})(\eta),
\end{aligned}
$$

which is enough to show that any random variable in $L^{2}$ can be expanded in a series of Wick polynomials showing that the Wick polynomials are an orthogonal basis of $L^{2}(\operatorname{law}(\eta))$ (but they are still not normalized). Indeed assume that $Z \in L^{2}(\operatorname{law}(\eta))$ but $Z \perp H\left(\psi_{i_{1}}, \ldots, \psi_{i_{n}}\right)(\eta)$ for all $n \geqslant 0$, $i_{1}, \ldots, i_{n} \in \mathbb{N}$, then

$$
\begin{aligned}
0 & =e^{\sum_{i} \sigma_{i}^{2}\left\|\psi_{i}\right\|^{2}} \mathbb{E}\left[Z\left(e^{\mathrm{D}_{\psi}^{*}} 1\right)(\eta)\right] \\
& =e^{\sum_{i} \sigma_{i}^{2}\left\|\psi_{i}\right\|^{2}} \mathbb{E}\left[Z e^{2 \sum_{i} \sigma_{i} \eta\left(\psi_{i}\right)-\sum_{i} \sigma_{i}^{2}\left\|\psi_{i}\right\|^{2}}\right] \\
& =\mathbb{E}\left[Z e^{2 \sum_{i} \sigma_{i} \eta\left(\psi_{i}\right)}\right] .
\end{aligned}
$$

Since the $\sigma_{i}$ are arbitrary, this means that $Z$ is orthogonal to any polynomial in $\eta$ (consider the derivatives in $\sigma \equiv 0$ ) and then that it is orthogonal also to $\exp \left(i \sum_{i} \sigma_{i} \eta\left(\psi_{i}\right)\right)$. So let $f \in \mathscr{S}\left(\mathbb{R}^{M}\right)$ and $\sigma_{i}=0$ for $i>m$, and observe that

$$
\begin{aligned}
0 & =(2 \pi)^{-m / 2} \int \mathrm{d} \sigma_{1} \cdots \mathrm{d} \sigma_{m} \mathscr{F} f\left(\sigma_{1}, \ldots, \sigma_{m}\right) \mathbb{E}\left[Z e^{i \sum_{i} \sigma_{i} \eta\left(\psi_{i}\right)}\right] \\
& =\mathbb{E}\left[Z f\left(\eta\left(\psi_{1}\right), \ldots, \eta\left(\psi_{M}\right)\right)\right]
\end{aligned}
$$

which means that $Z$ is orthogonal to all the random variables in $L^{2}$ which are measurable with respect to the $\sigma$-field generated by $\left(\eta\left(\psi_{n}\right)\right)_{n \geqslant 0}$. This implies $Z=0$. That is, Wick polynomials form a basis for $L^{2}(\operatorname{law}(\eta))$.

Example 2. The first few (un-normalized) Wick polynomials are

$$
\begin{gathered}
H\left(\psi_{i}\right)(\rho)=\mathrm{D}_{\psi_{i}}^{*} 1(\rho)=2 \rho\left(\psi_{i}\right) \\
H\left(\psi_{i}, \psi_{j}\right)(\rho)=\mathrm{D}_{\psi_{i}}^{*} \mathrm{D}_{\psi_{j}}^{*} 1=2 \mathrm{D}_{\psi_{i}}^{*} \rho\left(\psi_{j}\right)=-2 \delta_{i=j}+4 \rho\left(\psi_{i}\right) \rho\left(\psi_{j}\right),
\end{gathered}
$$

and

$$
\begin{aligned}
H\left(\psi_{i}, \psi_{j}, \psi_{k}\right)(\rho)= & \mathrm{D}_{\psi_{i}}^{*}\left(-2 \delta_{j=k}+4 \rho\left(\psi_{j}\right) \rho\left(\psi_{k}\right)\right) \\
= & -4 \delta_{j=k} \rho\left(\psi_{i}\right)-4 \delta_{i=j} \rho\left(\psi_{k}\right)-4 \delta_{i=k} \rho\left(\psi_{j}\right) \\
& +8 \rho\left(\psi_{i}\right) \rho\left(\psi_{j}\right) \rho\left(\psi_{k}\right) .
\end{aligned}
$$


Some other properties of Wick polynomials can be derived using the commutation relation between $\mathrm{D}$ and $\mathrm{D}^{*}$. By linearity $\mathrm{D}_{\varphi+\psi}^{*}=\mathrm{D}_{\varphi}^{*}+\mathrm{D}_{\psi}^{*}$, so that using the symmetry of $H$ we get

$$
H_{n}(\varphi+\psi):=H \underbrace{(\varphi+\psi, \ldots, \varphi+\psi)}_{n}=\sum_{0 \leqslant k \leqslant n}\left(\begin{array}{l}
n \\
k
\end{array}\right) H(\underbrace{\varphi, \ldots, \varphi}_{k}, \underbrace{\psi, \ldots, \psi}_{n-k}) .
$$

Then note that by Lemma 4 we have

$$
\begin{aligned}
\left(e^{\mathrm{D}_{\varphi}^{*}} 1\right)(\eta)\left(e^{\mathrm{D}_{\psi}^{*}} 1\right)(\eta) & =e^{2 \eta(\varphi)-\|\varphi\|^{2}} e^{2 \eta(\psi)-\|\psi\|^{2}}=e^{2 \eta(\varphi+\psi)-\|\varphi+\psi\|^{2}+2\langle\varphi, \psi\rangle} \\
& =\left(e^{\mathrm{D}_{\varphi+\psi}^{*}} 1\right)(\eta) e^{2\langle\varphi, \psi\rangle} .
\end{aligned}
$$

Expanding the exponentials,

$$
\begin{aligned}
\sum_{m, n} \frac{H_{m}(\varphi)}{m !} \frac{H_{n}(\psi)}{n !} & =\sum_{r, \ell} \frac{H_{r}(\varphi+\psi)}{r !} \frac{(2\langle\varphi, \psi\rangle)^{\ell}}{\ell !} \\
& =\sum_{p, q, \ell} \frac{H(\overbrace{\varphi, \ldots, \varphi}^{p}, \overbrace{\psi, \ldots, \psi}^{q})}{p ! q !} \frac{(2\langle\varphi, \psi\rangle)^{\ell}}{\ell !},
\end{aligned}
$$

and identifying the terms of the same homogeneity in $\varphi$ and $\psi$ respectively we get

$$
H_{m}(\varphi) H_{n}(\psi)=\sum_{p+\ell=m} \sum_{q+\ell=n} \frac{m ! n !}{p ! q ! \ell !} H(\overbrace{\varphi, \ldots, \varphi}^{p}, \overbrace{\psi, \ldots, \psi}^{q})(2\langle\varphi, \psi\rangle)^{\ell} .
$$

This gives a general formula for such products. By polarization of this multilinear form, we can also get a general formula for the products of general Wick polynomials. Indeed taking $\varphi=\sum_{i=1}^{m} \kappa_{i} \varphi_{i}$ and $\psi=$ $\sum_{j=1}^{n} \lambda_{j} \psi_{j}$ for arbitrary real coefficients $\kappa_{1}, \ldots, \kappa_{m}$ and $\lambda_{1}, \ldots, \lambda_{n}$, we have

$$
\begin{aligned}
& H_{m}\left(\sum_{i=1}^{m} \kappa_{i} \varphi_{i}\right) H_{n}\left(\sum_{j=1}^{n} \lambda_{j} \psi_{j}\right) \\
= & \sum_{i_{1}, \ldots, i_{m}} \sum_{j_{1}, \ldots, j_{n}} \kappa_{i_{1}} \cdots \kappa_{i_{m}} \lambda_{j_{1}} \cdots \lambda_{j_{m}} H\left(\varphi_{i_{1}}, \ldots, \varphi_{i_{m}}\right) H\left(\psi_{j_{1}}, \ldots, \psi_{j_{n}}\right) .
\end{aligned}
$$

Deriving this with respect to all the $\kappa, \lambda$ parameters and setting them to zero, we single out the term

$$
\begin{aligned}
& \sum_{\sigma \in S_{m}, \omega \in S_{n}} H\left(\varphi_{\sigma(1)}, \ldots, \varphi_{\sigma(m)}\right) H\left(\psi_{\omega(1)}, \ldots, \psi_{\omega(n)}\right) \\
= & m ! n ! H\left(\varphi_{1}, \ldots, \varphi_{m}\right) H\left(\psi_{1}, \ldots, \psi_{n}\right),
\end{aligned}
$$


where $S_{k}$ denotes the symmetric group on $\{1, \ldots, k\}$, and where we used the symmetry of the Wick polynomials. Doing the same also for the right hand side of (2.8) we get

$$
\begin{aligned}
& H\left(\varphi_{1}, \ldots, \varphi_{m}\right) H\left(\psi_{1}, \ldots, \psi_{n}\right) \\
= & \sum_{p+\ell=m} \sum_{q+\ell=n} \frac{1}{p ! q ! \ell !} \sum_{i, j} H(\overbrace{\varphi_{i_{1}}, \ldots, \varphi_{i_{p}}}^{p} \overbrace{\psi_{j_{1}}, \ldots, \psi_{j_{q}}}) \prod_{r=1}^{\ell}\left(2\left\langle\varphi_{i_{p+r}}, \psi_{j_{q+r}}\right\rangle\right),
\end{aligned}
$$

where the sum over $i, j$ runs over $i_{1}, \ldots, i_{m}$ permutation of $1, \ldots, m$ and similarly for $j_{1}, \ldots, j_{n}$. Since $H\left(\varphi_{i_{1}}, \ldots, \varphi_{i_{p}}, \psi_{j_{1}}, \ldots, \psi_{j_{q}}\right)(\eta)$ is orthogonal to 1 whenever $p+q>0$, we obtain in particular

$$
\begin{aligned}
\mathbb{E}\left[H\left(\psi_{1}, \ldots, \psi_{n}\right)(\eta) H\left(\psi_{1}, \ldots, \psi_{n}\right)(\eta)\right] & =\frac{1}{n !} \sum_{i, j} \prod_{r=1}^{n}\left(2\left\langle\psi_{i_{r}}, \psi_{j_{r}}\right\rangle\right) \\
& =\sum_{\sigma \in S_{n}} \prod_{r=1}^{n}\left(2\left\langle\psi_{r}, \psi_{\sigma(r)}\right\rangle\right) .
\end{aligned}
$$

In conclusion, we have shown that the family

$$
\left\{\left(\sum_{\sigma \in S_{n}} \prod_{r=1}^{n}\left(2\left\langle\psi_{r}, \psi_{\sigma(r)}\right\rangle\right)\right)^{-1 / 2} H\left(\psi_{i_{1}}, \ldots, \psi_{i_{n}}\right)(\eta): n \geqslant 0, i_{1}, \ldots, i_{n} \in \mathbb{N}\right\}
$$

is an orthonormal basis of $L^{2}(\operatorname{law}(\eta))$.

Remark 1. In our problem it will be convenient to take the Fourier basis as basis in the above computations. Let $e_{k}(x)=\exp (i k x) / \sqrt{2 \pi}=$ $a_{k}(x)+i b_{k}(x)$ where $\left(\sqrt{2} a_{k}\right)_{k \in \mathbb{N}}$ and $\left(\sqrt{2} b_{k}\right)_{k \in \mathbb{N}}$ form together a real valued orthonormal basis for $L^{2}(\mathbb{T})$. Then $\rho\left(e_{k}\right)^{*}=\rho\left(e_{-k}\right)$ whenever $\rho$ is real valued, and we will denote $\mathrm{D}_{k}=\mathrm{D}_{e_{k}}=\mathrm{D}_{a_{k}}+i \mathrm{D}_{b_{k}}$ and similarly for $\mathrm{D}_{k}^{*}=\mathrm{D}_{a_{k}}^{*}-i \mathrm{D}_{b_{k}}^{*}=-\mathrm{D}_{-k}+2 \rho\left(e_{-k}\right)$. In this way, $\mathrm{D}_{k}^{*}$ is the adjoint of $\mathrm{D}_{k}$ with respect to the Hermitian scalar product on $L^{2}(\Omega ; \mathbb{C})$ and the Ornstein-Uhlenbeck generator takes the form

$$
L_{0}=\sum_{k \in \mathbb{N}}\left(\mathrm{D}_{\partial_{x} a_{k}}^{*} \mathrm{D}_{\partial_{x} a_{k}}+\mathrm{D}_{\partial_{x} b_{k}}^{*} \mathrm{D}_{\partial_{x} b_{k}}\right)=\frac{1}{2} \sum_{k \in \mathbb{Z}} k^{2} \mathrm{D}_{k}^{*} \mathrm{D}_{k}
$$

(convince yourself of the last identity by observing that $D_{k}^{*} D_{k}+D_{-k}^{*} D_{-k}=$ $\left.2\left(\mathrm{D}_{a_{k}}^{*} \mathrm{D}_{a_{k}}+\mathrm{D}_{b_{k}}^{*} \mathrm{D}_{b_{k}}\right) !\right)$. Similarly,

$$
\mathcal{E}(F, G)=\sum_{k \in \mathbb{Z}} k^{2}\left(\mathrm{D}_{k} F\right)^{*}\left(\mathrm{D}_{k} G\right)
$$




\subsection{The Itô trick}

We are ready now to start our computations. Recall that we want to analyse $J_{t}(\varphi)=\int_{0}^{t} \partial_{x} X_{s}^{2}(\varphi) \mathrm{d} s$ using Itô calculus with respect to the Ornstein-Uhlenbeck process. We want to understand $J_{t}$ as a correction term in Itô's formula: if we can find a function $G$ such that $L_{0} G\left(X_{t}\right)=$ $\partial_{x} X_{t}^{2}$, then we get from Itô's formula

$$
\int_{0}^{t} \partial_{x} X_{s}^{2} \mathrm{~d} s=G\left(X_{t}\right)-G\left(X_{0}\right)-M_{G, t}
$$

where $M_{G}$ is a martingale depending on $G$. Of course, $G$ will not be a cylindrical function but we only defined $L_{0}$ on cylindrical functions. So to make the following calculations rigorous we would again have to replace $\partial_{x} X_{t}^{2}$ by $\partial_{x} \Pi_{n} X_{t}^{2}$ and then pass to the limit, see the paper [15] for details. As before we will perform the calculations already in the limit $N=+\infty$, in order to simplify the computations and not to obscure the ideas through technicalities. The next problem is that the pointwise evaluation $\int_{0}^{t} \partial_{x} X_{s}^{2}(x) \mathrm{d} s$ does not make any sense because the integral will only be defined as a space distribution. So we will consider

$$
G: \mathscr{S}^{\prime} \rightarrow \mathscr{S}^{\prime}
$$

instead of $G: \mathscr{S}^{\prime} \rightarrow \mathbb{C}$. Note however that we can reduce every such $G$ to a function from $\mathscr{S}^{\prime}$ to $\mathbb{C}$ by considering $\rho \mapsto G(\rho)\left(e_{k}\right)$ for all $k$.

Now for a fixed $k$, we have

$$
\partial_{x} X_{t}^{2}\left(e_{k}\right)=\frac{i k}{\sqrt{2}} \sum_{\ell+m=k} X_{t}\left(e_{\ell}\right) X_{t}\left(e_{m}\right)=\frac{i k}{\sqrt{2}} \sum_{\ell+m=k} H_{\ell, m}\left(X_{t}\right),
$$

where $H_{\ell, m}(\rho)=\frac{1}{4}\left(\mathrm{D}_{-\ell}^{*} \mathrm{D}_{-m}^{*} 1\right)(\rho)=\rho\left(e_{\ell}\right) \rho\left(e_{m}\right)-\frac{1}{2} \delta_{\ell+m=0}$ is a second order Wick polynomial so that $L_{0} H_{\ell, m}=-\left(\ell^{2}+m^{2}\right) H_{\ell, m}$ by $(2.7)$. Therefore, it is enough to take

$$
G\left(X_{t}\right)\left(e_{k}\right)=-i k \sum_{\ell+m=k} \frac{H_{\ell, m}\left(X_{t}\right)}{\ell^{2}+m^{2}}
$$

This corresponds to the distribution $G\left(X_{t}\right)(\varphi)=-\int_{0}^{\infty} \partial_{x}\left(e^{s \Delta} X_{t}\right)^{2}(\varphi) \mathrm{d} s$ (check it!). Then

$$
G\left(X_{t}\right)(\varphi)=G\left(X_{0}\right)(\varphi)+M_{G, t}(\varphi)+J_{t}(\varphi)
$$

where $M_{G, t}(\varphi)$ is a martingale with quadratic variation

$$
\mathrm{d}\left\langle M_{G, *}(\varphi), M_{G, *}(\varphi)\right\rangle_{t}=\mathcal{E}(G(*)(\varphi), G(*)(\varphi))\left(X_{t}\right) \mathrm{d} t .
$$


We can estimate

$\mathbb{E}\left[\left|J_{t}(\varphi)-J_{s}(\varphi)\right|^{2 p}\right] \lesssim_{p} \mathbb{E}\left[\left|M_{G, t}(\varphi)-M_{G, s}(\varphi)\right|^{2 p}\right]+\mathbb{E}\left[\left|G\left(X_{t}\right)(\varphi)-G\left(X_{s}\right)(\varphi)\right|^{2 p}\right]$.

To bound the martingale expectation, we will use the following Burkholder inequality:

Lemma 5. Let $m$ be a continuous local martingale with $m_{0}=0$. Then for all $T \geqslant 0$ and $p>1$,

$$
\mathbb{E}\left[\sup _{t \leqslant T}\left|m_{t}\right|^{2 p}\right] \leqslant C_{p} \mathbb{E}\left[\langle m\rangle_{T}^{p}\right] .
$$

Proof. Start by assuming that $m$ and $\langle m\rangle$ are bounded. Itô's formula yields

$$
\mathrm{d}\left|m_{t}\right|^{2 p}=(2 p)\left|m_{t}\right|^{2 p-1} \mathrm{~d} m_{t}+\frac{1}{2}(2 p)(2 p-1)\left|m_{t}\right|^{2 p-2} \mathrm{~d}\langle m\rangle_{t},
$$

and therefore

$$
\mathbb{E}\left[\left|m_{T}\right|^{2 p}\right]=C_{p} \mathbb{E}\left[\int_{0}^{T}\left|m_{s}\right|^{2 p-2} \mathrm{~d}\langle m\rangle_{s}\right] \leqslant C_{p} \mathbb{E}\left[\sup _{t \leqslant T}\left|m_{t}\right|^{2 p-2}\langle m\rangle_{T}\right] .
$$

By the Cauchy-Schwarz inequality we get

$$
\mathbb{E}\left[\left|m_{T}\right|^{2 p}\right] \leqslant C_{p} \mathbb{E}\left[\sup _{t \leqslant T}\left|m_{t}\right|^{2 p}\right]^{(2 p-2) / 2 p} \mathbb{E}\left[\langle m\rangle_{T}^{p}\right]^{1 / p} .
$$

But now Doob's $L^{p}$ inequality yields $\mathbb{E}\left[\sup _{t \leqslant T}\left|m_{t}\right|^{2 p}\right] \leqslant C_{p}^{\prime} \mathbb{E}\left[\left|m_{T}\right|^{2 p}\right]$, and this implies the claim in the bounded case. The unbounded case can be treated with a localization argument.

Applying Burkholder's inequality, we obtain

$$
\begin{aligned}
\mathbb{E}\left[\left|J_{t}(\varphi)-J_{s}(\varphi)\right|^{2 p}\right] \lesssim_{p} \mathbb{E}\left[\left|\int_{s}^{t} \mathcal{E}(G(*)(\varphi), G(*)(\varphi))\left(X_{r}\right) \mathrm{d} r\right|^{p}\right] \\
+\mathbb{E}\left[\left|G\left(X_{t}\right)(\varphi)-G\left(X_{s}\right)(\varphi)\right|^{2 p}\right] \\
\leqslant(t-s)^{p-1} \int_{s}^{t} \mathbb{E}\left[\left|\mathcal{E}(G(*)(\varphi), G(*)(\varphi))\left(X_{r}\right)\right|^{p}\right] \mathrm{d} r \\
\quad+\mathbb{E}\left[\left|G\left(X_{t}\right)(\varphi)-G\left(X_{s}\right)(\varphi)\right|^{2 p}\right] \\
=(t-s)^{p} \mathbb{E}\left[|\mathcal{E}(G(*)(\varphi), G(*)(\varphi))(\eta)|^{p}\right] \\
+\mathbb{E}\left[\left|G\left(X_{t}\right)(\varphi)-G\left(X_{s}\right)(\varphi)\right|^{2 p}\right],
\end{aligned}
$$

using that $X_{r} \sim \eta$. Now

$$
\mathrm{D}_{m} G(\rho)\left(e_{k}\right)=-2 i k \frac{\rho\left(e_{k-m}\right)}{(k-m)^{2}+m^{2}},
$$


and therefore

$$
\begin{aligned}
\mathcal{E}\left(G(*)\left(e_{k}\right), G(*)\left(e_{k}\right)\right)(\rho) & =\sum_{m} m^{2} \mathrm{D}_{-m} G(\rho)\left(e_{-k}\right) \mathrm{D}_{m} G(\rho)\left(e_{k}\right) \\
& =4 k^{2} \sum_{\ell+m=k} m^{2} \frac{\left|\rho\left(e_{\ell}\right)\right|^{2}}{\left(\ell^{2}+m^{2}\right)^{2}} \\
& \lesssim k^{2} \sum_{\ell+m=k} \frac{\left|\rho\left(e_{\ell}\right)\right|^{2}}{\ell^{2}+m^{2}}
\end{aligned}
$$

which implies that

$$
\begin{aligned}
\mathbb{E}\left[\left|\mathcal{E}\left(G(*)\left(e_{k}\right), G(*)\left(e_{k}\right)\right)(\eta)\right|\right] & \lesssim k^{2} \mathbb{E} \sum_{\ell+m=k} \frac{\left|\eta\left(e_{\ell}\right)\right|^{2}}{\ell^{2}+m^{2}} \\
& \lesssim k^{2} \sum_{\ell+m=k} \frac{1}{\ell^{2}+m^{2}} \\
& \lesssim|k| .
\end{aligned}
$$

A similar computation gives also that

$$
\mathbb{E}\left[\left|\mathcal{E}\left(G(*)\left(e_{k}\right), G(*)\left(e_{k}\right)\right)(\eta)\right|^{p}\right] \lesssim|k|^{p} .
$$

Further, we have

$$
\begin{aligned}
\mathbb{E}\left[\left|G\left(X_{t}\right)\left(e_{k}\right)-G\left(X_{s}\right)\left(e_{k}\right)\right|^{2}\right] & \lesssim k^{2} \sum_{\ell+m=k} \mathbb{E}\left[\frac{\left.\mid H_{\ell, m}\left(X_{t}\right)-H_{\ell, m}\left(X_{s}\right)\right)^{2}}{\left(\ell^{2}+\left.m^{2}\right|^{2}\right.}\right] \\
& \lesssim k^{2}|t-s| \sum_{\ell+m=k} \frac{m^{2}}{\left(\ell^{2}+m^{2}\right)^{2}} \lesssim|k||t-s| .
\end{aligned}
$$

And finally, since $G$ is a second order polynomial of a Gaussian process we can apply once more Gaussian hypercontractivity to obtain

$$
\mathbb{E}\left[\left|J_{t}\left(e_{k}\right)-J_{s}\left(e_{k}\right)\right|^{2 p}\right] \lesssim_{p}(t-s)^{p}|k|^{p} .
$$

The advantage of the Itô trick with respect to the explicit Gaussian computation is that it goes over to the non-Gaussian case. Indeed note that while the boundary term $G\left(X_{t}\right)(\varphi)-G\left(X_{s}\right)(\varphi)$ has been estimated using a lot of the Gaussian information about $X$, we used only the law at a fixed time to handle the term $\int_{s}^{t} \mathcal{E}(G(*)(\varphi), G(*)(\varphi))\left(X_{r}\right) \mathrm{d} r$.

In order to carry over these computation to the solution of the non-linear dynamics $u$, we need to replace the generator of $X$ with that of $u$ and to have a way to handle the boundary terms. The idea is now to reverse the Markov process $u$ in time, which will allow us to kill the antisymmetric 
part of the generator and at the same time kill the boundary terms. Indeed observe that if $u$ solves the stochastic Burgers equation, then formally we have the Itô formula

$$
\mathrm{d}_{t} F\left(u_{t}\right)=\sum_{i=1}^{n} F_{i}\left(u_{t}\right) \mathrm{d} M_{t}\left(\varphi_{i}\right)+L F\left(u_{t}\right) \mathrm{d} t
$$

where $L$ is now the full generator of the non-linear dynamics, given by

$$
L F(\rho)=L_{0} F(\rho)+\sum_{i} F_{i}(\rho)\left\langle\partial_{x} \rho^{2}, \varphi_{i}\right\rangle=L_{0} F(\rho)+B F(\rho),
$$

where

$$
B F(\rho)=\sum_{k}\left(\partial_{x} \rho^{2}\right)\left(e_{k}\right) \mathrm{D}_{k} F(\rho) .
$$

Formally, the non-linear term is antisymmetric with respect to the invariant measure of $L_{0}$. Indeed since $B$ is a first order operator

$\mathbb{E}[(B F(\eta)) G(\eta)]=\mathbb{E}[(B(F G)(\eta))]-\mathbb{E}[F(\eta)(B G(\eta))]=-\mathbb{E}[F(\eta)(B G(\eta))]$

provided $\mathbb{E}[B F(\eta)]=0$ for any cylinder function $F$. Let us show this. We have

$$
\begin{aligned}
\mathbb{E}[B F(\eta)] & =\sum_{k} \mathbb{E}\left[\left(\partial_{x} \eta^{2}\right)\left(e_{k}\right) \mathrm{D}_{k} F(\eta)\right] \\
& =-\sum_{k} \mathbb{E}\left[\left(\mathrm{D}_{k}\left(\partial_{x} \eta^{2}\right)\left(e_{k}\right)\right) F(\eta)\right]+\sum_{k} \mathbb{E}\left[\mathrm{D}_{k}\left[\left(\partial_{x} \eta^{2}\right)\left(e_{k}\right) F(\eta)\right]\right]
\end{aligned}
$$

But now we get from $(2.11)$

$$
\mathrm{D}_{k}\left(\partial_{x} \eta^{2}\right)\left(e_{k}\right)=\sqrt{2} i k \eta\left(e_{0}\right)=\pi^{-1 / 2} i k\langle\eta, 1\rangle=0,
$$

where we used that $\langle\eta, 1\rangle=0$. Gaussian integration by parts then formally gives

$$
\begin{aligned}
\mathbb{E}[B F(\eta)] & =\sum_{k} \mathbb{E}\left[\mathrm{D}_{k}\left[\left(\partial_{x} \eta^{2}\right)\left(e_{k}\right) F(\eta)\right]\right]=\sum_{k} \mathbb{E}\left[\eta\left(e_{k}\right)\left(\partial_{x} \eta^{2}\right)\left(e_{k}\right) F(\eta)\right] \\
& =\mathbb{E}\left[\left\langle\eta, \partial_{x} \eta^{2}\right\rangle F(\eta)\right]=\frac{1}{3} \mathbb{E}\left[\left\langle 1, \partial_{x} \eta^{3}\right\rangle F(\eta)\right]=0
\end{aligned}
$$

since $\left\langle 1, \partial_{x} \eta^{3}\right\rangle=-\left\langle\partial_{x} 1, \eta^{3}\right\rangle=0$ (but of course $\left\langle\eta, \partial_{x} \eta^{2}\right\rangle$ is not well defined).

The dynamics of $u$ backwards in time has a Markovian description which is the subject of the next exercise. 
Exercise 7. Let $\left(y_{t}\right)_{t \geqslant 0}$ be a stationary Markov process on a Polish space, with semigroup $\left(P_{t}\right)_{t \geqslant 0}$ and stationary distribution $\mu$. Show that if $P_{t}^{*}$ is the adjoint of $P_{t}$ in $L^{2}(\mu)$, then $\left(P_{t}^{*}\right)$ is a semigroup of operators on $L^{2}(\mu)$ (that is $P_{0}^{*}=\mathrm{id}$ and $P_{s+t}^{*}=P_{s}^{*} P_{t}^{*}$ as operators on $L^{2}(\mu)$ ). Show that if $y_{0} \sim \mu$, then for all $T>0$ the process $\hat{y}_{t}=y_{T-t}, t \in[0, T]$, is also Markov, with semigroup $\left(P_{t}^{*}\right)_{t \in[0, T]}$, and that $\mu$ is also an invariant distribution for $\left(P_{t}^{*}\right)$. Show also that if $\left(P_{t}\right)$ has generator $L$ then $\left(P_{t}^{*}\right)$ has generator $L^{*}$ which is the adjoint of $L$ with respect to $L^{2}(\mu)$.

Now if we reverse the process in time letting $\hat{u}_{t}=u_{T-t}$, we have by stationarity

$$
\mathbb{E}\left[F\left(\hat{u}_{t}\right) G\left(\hat{u}_{0}\right)\right]=\mathbb{E}\left[F\left(u_{T-t}\right) G\left(u_{T}\right)\right]=\mathbb{E}\left[F\left(u_{0}\right) G\left(u_{t}\right)\right] .
$$

So if we denote by $\hat{L}$ the generator of $\hat{u}$ :

$$
\begin{aligned}
\mathbb{E}\left[\hat{L} F\left(\hat{u}_{0}\right) G\left(\hat{u}_{0}\right)\right] & =\left.\frac{\mathrm{d}}{\mathrm{d} t}\right|_{t=0} \mathbb{E}\left[F\left(\hat{u}_{t}\right) G\left(\hat{u}_{0}\right)\right] \\
& =\left.\frac{\mathrm{d}}{\mathrm{d} t}\right|_{t=0} \mathbb{E}\left[F\left(u_{0}\right) G\left(u_{t}\right)\right] \\
& =\mathbb{E}\left[L G\left(u_{0}\right) F\left(u_{0}\right)\right],
\end{aligned}
$$

which means that $\hat{L}$ is the adjoint of $L$, that is

$$
\hat{L} F(\rho)=L_{0} F(\rho)-B F(\rho)=L_{0} F(\rho)-\sum_{k}\left(\partial_{x} \rho^{2}\right)\left(e_{k}\right) \mathrm{D}_{k} F(\rho) .
$$

In other words, the reversed process solves

$$
\hat{u}_{t}(\varphi)=\hat{u}_{0}(\varphi)+\int_{0}^{t} \hat{u}_{s}(\Delta \varphi) \mathrm{d} s+\int_{0}^{t}\left\langle\hat{u}_{s}^{2}, \partial_{x} \varphi\right\rangle \mathrm{d} s-\int_{0}^{t} \hat{\xi}_{s}\left(\partial_{x} \varphi\right) \mathrm{d} s
$$

for a different space-time white noise $\hat{\xi}$. Then Itô's formula for $\hat{u}$ gives

$$
\mathrm{d}_{t} F\left(\hat{u}_{t}\right)=\sum_{i=1}^{n} F_{i}\left(\hat{u}_{t}\right) \mathrm{d} \hat{M}_{t}\left(\varphi_{i}\right)+\hat{L} F\left(\hat{u}_{t}\right) \mathrm{d} t
$$

where for all test functions $\varphi$, the process $\hat{M}(\varphi)$ is a martingale in the filtration of $\hat{u}$ with covariance

$$
\mathrm{d}\langle\hat{M}(\varphi), \hat{M}(\psi)\rangle_{t}=\left\langle\partial_{x} \varphi, \partial_{x} \psi\right\rangle_{L^{2}(\mathbb{T})} \mathrm{d} t
$$

Combining the Itô formulas for $u$ and $\hat{u}$, we get

$$
F\left(u_{T}\right)(\varphi)=F\left(u_{0}\right)(\varphi)+M_{F, T}(\varphi)+\int_{0}^{T} L F\left(u_{s}\right)(\varphi) \mathrm{d} s
$$


and

$$
\begin{aligned}
F\left(u_{0}\right)(\varphi) & =F\left(\hat{u}_{T}\right)(\varphi)=F\left(\hat{u}_{0}\right)(\varphi)+\hat{M}_{F, T}(\varphi)+\int_{0}^{T} \hat{L} F\left(\hat{u}_{s}\right)(\varphi) \mathrm{d} s \\
& =F\left(u_{T}\right)(\varphi)+\hat{M}_{F, T}(\varphi)+\int_{0}^{T} \hat{L} F\left(u_{s}\right)(\varphi) \mathrm{d} s
\end{aligned}
$$

and summing up these two equalities gives

$$
0=M_{F, T}(\varphi)+\hat{M}_{F, T}(\varphi)+\int_{0}^{T}(\hat{L}+L) F\left(u_{s}\right)(\varphi) \mathrm{d} s
$$

that is

$$
2 \int_{0}^{T} L_{0} F\left(u_{s}\right)(\varphi) \mathrm{d} s=-M_{F, T}(\varphi)-\hat{M}_{F, T}(\varphi) .
$$

An added benefit of this forward-backward representation is that the only term which required quite a lot of informations about $X$, that is the boundary term $F\left(X_{t}\right)(\varphi)-F\left(X_{s}\right)(\varphi)$ does not appear at all now. As above if $2 L_{0} F(\rho)=\partial_{x} \rho^{2}$, we end up with

$$
\int_{0}^{T} \partial_{x} u_{s}^{2}(\varphi) \mathrm{d} s=-M_{F, T}(\varphi)-\hat{M}_{F, T}(\varphi) .
$$

Exercise 8. Perform a similar formal calculation as in (2.13) to see that $\mathbb{E}[L F(\eta)]=0$ for all cylindrical functions $F$, so that $\eta$ should also be invariant for the stochastic Burgers equation. Combine this with (2.14) to show that setting $\mathcal{N}_{t}^{N}(\varphi)=\int_{0}^{t} \partial_{x}\left(\Pi_{N} u_{s}\right)^{2}(\varphi) \mathrm{d} s$ we have

$$
\mathbb{E}\left[\left|\mathcal{N}_{t}^{N}\left(e_{k}\right)-\mathcal{N}_{s}^{N}\left(e_{k}\right)\right|^{2 p}\right] \lesssim p(t-s)^{p}|k|^{p}
$$

and letting $\mathcal{N}_{t}^{N, M}=\mathcal{N}_{t}^{N}-\mathcal{N}_{t}^{M}$ we get

$$
\mathbb{E}\left[\left|\mathcal{N}_{t}^{N, M}\left(e_{k}\right)-\mathcal{N}_{s}^{N, M}\left(e_{k}\right)\right|^{2 p}\right] \lesssim_{p}(|k| / N)^{\varepsilon p}(t-s)^{p}|k|^{p}
$$

for all $1 \leqslant N \leqslant M$. Use this to derive that

$$
\left(\mathbb{E}\left[\left\|\mathcal{N}_{t}^{N, M}-\mathcal{N}_{s}^{N, M}\right\|_{H^{\alpha}}^{2 p}\right]\right)^{1 / 2 p} \lesssim_{p, \alpha} N^{-\varepsilon / 2}(t-s)^{1 / 2}
$$

for all $\alpha<-1-\varepsilon$, and realize that this estimate allows you to prove compactness of the approximations $\mathcal{N}^{N}$ and then convergence to a limit $\mathcal{N}$ in $L^{2 p}\left(\Omega ; C^{1 / 2-} H^{-1-}\right)$. 


\subsection{Controlled distributions}

Let us cook up a definition which will allow us to rigorously perform the formal computations above in a general setting.

Definition 2. Let $u, \mathcal{A}: \mathbb{R}_{+} \times \mathbb{T} \rightarrow \mathscr{S}^{\prime}(\mathbb{T})$ be a couple of generalized (i.e. distribution-valued) processes such that

i. For all $\varphi \in \mathscr{S}(\mathbb{T})$ the process $t \mapsto u_{t}(\varphi)$ is a continuous semimartingale satisfying

$$
u_{t}(\varphi)=u_{0}(\varphi)+\int_{0}^{t} u_{s}(\Delta \varphi) \mathrm{d} s+\mathcal{A}_{t}(\varphi)+M_{t}(\varphi)
$$

where $t \mapsto M_{t}(\varphi)$ is a martingale with quadratic variation $\langle M(\varphi), M(\psi)\rangle_{t}=\left\langle\partial_{x} \varphi, \partial_{x} \psi\right\rangle_{L^{2}(\mathbb{T})} t$ and $t \mapsto \mathcal{A}_{t}(\varphi)$ is a finite variation process with $\mathcal{A}_{0}(\varphi)=0$.

ii. For all $t \geqslant 0$ the random distribution $\varphi \mapsto u_{t}(\varphi)$ is a zero mean space white noise with variance $\|\varphi\|_{L_{0}^{2}}^{2} / 2$.

iii. For any $T>0$ the reversed process $\hat{u}_{t}=u_{T-t}$ has again properties $i$, ii with martingale $\hat{M}$ and finite variation part $\hat{\mathcal{A}}$ such that $\hat{\mathcal{A}}_{t}(\varphi)=$ $-\left(\mathcal{A}_{T}(\varphi)-\mathcal{A}_{T-t}(\varphi)\right)$.

Any pair of processes $(u, \mathcal{A})$ satisfying these condition will be called controlled by the Ornstein-Uhlenbeck process and we will denote the set of all such processes with $\mathcal{Q}_{\mathrm{ou}}$.

Theorem 2 ([15], Lemma 1). Assume that $(u, \mathcal{A}) \in \mathcal{Q}_{\text {ou }}$ and for any $N \geqslant 1, t \geqslant 0, \varphi \in \mathscr{S}$ let

$$
\mathcal{N}_{t}^{N}(\varphi)=\int_{0}^{t} \partial_{x}\left(\Pi_{N} u_{s}\right)^{2}(\varphi) \mathrm{d} s
$$

Then for any $p \geqslant 1\left(\mathcal{N}^{N}\right)_{N \geqslant 1}$ converges in $L^{p}(\Omega)$ to a space-time distribution $\mathcal{N} \in C^{1 / 2-} H^{-1-}$.

We are now at a point where we can give a meaning to our original equation.

Definition 3. A pair of random distribution $(u, \mathcal{A}) \in \mathcal{Q}_{\text {ou }}$ is an energy solution to the stochastic Burgers equation if it satisfies

$$
u_{t}(\varphi)=u_{0}(\varphi)+\int_{0}^{t} u_{s}(\Delta \varphi) \mathrm{d} s+\mathcal{N}_{t}(\varphi)+M_{t}(\varphi)
$$

for all $t \geqslant 0$ and $\varphi \in \mathscr{S}$. That is if $\mathcal{A}=\mathcal{N}$. 
Now we are in a relatively standard setting of needing to prove existence and uniqueness of such energy solutions. Note that in general the solutions are pairs of processes $(u, \mathcal{A})$.

Remark 2. The notion of energy solution has been introduced (in a slightly different way) in the work of Gonçalves and Jara [11] on macroscopic universal fluctuations of weakly asymmetric interacting particle systems.

\subsection{Existence of solutions}

For the existence, the way to proceed is quite standard. We approximate the equation, construct approximate solutions and then try to have enough compactness to have limiting points which then naturally will satisfy the requirements for energy solutions. For any $N \geqslant 1$ consider solutions $u^{N}$ to

$$
\partial_{t} u^{N}=\Delta u^{N}+\partial_{x} \Pi_{N}\left(\Pi_{N} u^{N}\right)^{2}+\partial_{x} \xi
$$

These are generalized functions such that

$$
\mathrm{d} u_{t}^{N}\left(e_{k}\right)=-k^{2} u_{t}^{N}\left(e_{k}\right) \mathrm{d} t+\left[\partial_{x} \Pi_{N}\left(\Pi_{N} u^{N}\right)^{2}\right]\left(e_{k}\right) \mathrm{d} t+i k \mathrm{~d} \beta_{t}(k)
$$

for $k \in \mathbb{Z}$ and $t \geqslant 0$. We take $u_{0}$ to be the white noise with covariance $u_{0}(\varphi) \sim \mathcal{N}\left(0,\|\varphi\|^{2} / 2\right)$. The point of our choice of the non-linearity is that this (infinite-dimensional) system of equations decomposes into a finite dimensional system for $\left(v^{N}(k)=\Pi_{N} u^{N}\left(e_{k}\right)\right)_{k:|k| \leqslant N}$ and an infinite number of one-dimensional equations for each $u^{N}\left(e_{k}\right)$ with $|k|>N$. Indeed if $|k|>N$ we have $\left[\partial_{x} \Pi_{N}\left(\Pi_{N} u^{N}\right)^{2}\right]\left(e_{k}\right)=0$ so $u_{t}\left(e_{k}\right)=X_{t}\left(e_{k}\right)$ the Ornstein-Uhlenbeck process with initial condition $X_{0}\left(e_{k}\right)=u_{0}\left(e_{k}\right)$ which renders it stationary in time (check it). The equation for $\left(v^{N}(k)\right)_{|k| \leqslant N}$ reads

$$
\mathrm{d} v_{t}^{N}(k)=-k^{2} v_{t}^{N}(k) \mathrm{d} t+b_{k}\left(v_{t}^{N}\right) \mathrm{d} t+i k \mathrm{~d} \beta_{t}(k), \quad|k| \leqslant N, t \geqslant 0
$$

where

$$
b_{k}\left(v_{t}^{N}\right)=i k \sum_{\ell+m=k} \mathbb{I}_{|\ell|,|k|,|m| \leqslant N} v_{t}^{N}(\ell) v_{t}^{N}(m) .
$$

This is a standard finite-dimensional ODE having global solutions for all initial conditions which gives rise to a nice Markov process. The fact that solutions do not blow up even if the interaction is quadratic can be seen by computing the evolution of the norm

$$
A_{t}=\sum_{|k| \leqslant N}\left|v_{t}^{N}(k)\right|^{2}
$$


and by showing that

$$
\begin{aligned}
\mathrm{d} A_{t}= & 2 \sum_{|k| \leqslant N} v_{t}^{N}(-k) \mathrm{d} v_{t}^{N}(k) \\
= & -2 \sum_{|k| \leq N} k^{2}\left|v_{t}^{N}(k)\right|^{2} \mathrm{~d} t+2 \sum_{|k| \leqslant N} v_{t}^{N}(-k) b_{k}\left(v_{t}^{N}\right) \mathrm{d} t \\
& +2 i k \sum_{|k| \leqslant N} v_{t}^{N}(-k) \mathrm{d} \beta_{t}(k) .
\end{aligned}
$$

Since $A$ is nonnegative, we increase its absolute value by omitting the first contribution. But now

$$
\begin{aligned}
\sum_{|k| \leqslant N} v_{t}^{N}(-k) b_{k}\left(v_{t}^{N}\right) & =2 i \sum_{k, \ell, m: \ell+m=k} \mathbb{I}_{|\ell|,|k|,|m| \leqslant N} k v_{t}^{N}(\ell) v_{t}^{N}(m) v_{t}^{N}(-k) \\
& =-2 i \sum_{k, \ell, m: \ell+m+k=0} \mathbb{I}_{|\ell|,|k|,|m| \leqslant N}(k) v_{t}^{N}(\ell) v_{t}^{N}(m) v_{t}^{N}(k)
\end{aligned}
$$

and by symmetry of this expression it is equal to

$$
-\frac{2}{3} i \sum_{k, \ell, m: \ell+m+k=0} \mathbb{I}_{|\ell|,|k|,|m| \leqslant N}(k+\ell+m) v_{t}^{N}(\ell) v_{t}^{N}(m) v_{t}^{N}(k)=0,
$$

so $\left|A_{t}\right| \leq\left|A_{0}+M_{t}\right|$ where $\mathrm{d} M_{t}=2 \sum_{|k| \leqslant N} \mathbb{I}_{|k| \leqslant N}(i k) v_{t}^{N}(-k) \mathrm{d} \beta_{t}(k)$. Now

$$
\mathbb{E}\left[M_{T}^{2}\right] \lesssim \int_{0}^{T} \sum_{|k| \leqslant N} k^{2}\left|v_{t}^{N}(k)\right|^{2} \mathrm{~d} t \lesssim N^{2} \int_{0}^{T} A_{t} \mathrm{~d} t
$$

and then by martingales inequalities

$$
\begin{aligned}
\mathbb{E}\left[\sup _{t \in[0, T]}\left(A_{t}\right)^{2}\right] & \leqslant 2 \mathbb{E}\left[A_{0}^{2}\right]+2 \mathbb{E}\left[\sup _{t \in[0, T]}\left(M_{t}\right)^{2}\right] \leqslant 2 \mathbb{E}\left[A_{0}^{2}\right]+8 \mathbb{E}\left[M_{T}^{2}\right] \\
& \leqslant 2 \mathbb{E}\left[A_{0}^{2}\right]+C N^{2} \int_{0}^{T} \mathbb{E}\left(A_{t}\right) \mathrm{d} t .
\end{aligned}
$$

Now Gronwall's inequality gives

$$
\mathbb{E}\left[\sup _{t \in[0, T]}\left(A_{t}\right)^{2}\right] \lesssim e^{C N^{2} T} \mathbb{E}\left[A_{0}^{2}\right],
$$

from where we can deduce (by a continuation argument) that almost surely there is no blowup at finite time for the dynamics. The generator $L^{N}$ for the Galerkin dynamics is given by

$$
L^{N} F(\rho)=L_{0} F(\rho)+B^{N} F(\rho),
$$


where

$$
B^{N} F(\rho)=\sum_{k} \mathbb{I}_{|k| \leqslant N}\left(\partial_{x} \rho^{2}\right)\left(e_{k}\right) \mathrm{D}_{k} F(\rho) .
$$

And again the non-linear drift $B^{N}$ is antisymmetric with respect to the invariant measure of $L_{0}$ by a computation similar to that for the full drift $B$. Next, using Echeverría's criterion [7] we can obtain the invariance of the white noise from its infinitesimal invariance which can be checked at the level of the generator $L^{N}$. Finally it is also possible to rigorously show that the reversed process is a Markov process with generator

$$
\hat{L}^{N} F(\rho)=L_{0} F(\rho)-B^{N} F(\rho),
$$

thus proving that the reversed non-linear drift is the opposite of the forward one. Taking

$$
\mathcal{A}_{t}^{N}\left(e_{k}\right)=\int_{0}^{t} b_{k}\left(v_{s}^{N}\right) d s
$$

we obtain that $\left(v^{N}, \mathcal{A}^{N}\right) \in \mathcal{Q}_{\mathrm{ou}}$. Note that this result depends on the fact that we kept the full linear part $L_{0}$ of the generator. A more standard Galerkin truncation would have lead us to a process which is controlled by the Galerkin-truncated OU process. Estimates would have resulted in a similar way but our setup is simpler.

Given that $\left(v^{N}, \mathcal{A}^{N}\right)$ is controlled by the OU process, the Itô trick applied to $\mathcal{A}^{N}$ provides enough compactness in order to pass to the limit as $N \rightarrow \infty$ and build an energy solution to the Stochastic Burgers equation. See [15] for additional details on the limiting procedure and [30] for details on how to implement the Itô trick on the level of diffusions.

Remark 3. There is however one small catch: For a controlled distribution $(u, \mathcal{A})$ we required $\mathcal{A}(\varphi)$ to be of finite variation for every test function $\varphi$. The solution $\left(v^{N}, \mathcal{A}^{N}\right)$ to the truncated equation will satisfy this, but in the limit $\mathcal{A}(\varphi)$ will only have vanishing quadratic variation and it will not be of finite variation (in other words $u(\varphi)$ is a Dirichlet process and not a semimartingale). Luckily in this setting it is still possible to derive an Itô formula and everything goes through as described above, see [15] for details. 


\section{Chapter 3}

\section{Besov spaces}

Here we collect some classical results from harmonic analysis which we will need in the following. We concentrate on distributions and SPDEs on the torus, but everything in this Section applies mutatis mutandis on the full space $\mathbb{R}^{d}$, see [14]. The only problem is that then the stochastic terms will no longer be in the Besov spaces $\mathscr{C}^{\alpha}$ which we encounter below but rather in weighted Besov spaces. Handling SPDEs in weighted function spaces is more delicate and we prefer here to concentrate on the simpler situation of the torus.

We will use Littlewood-Paley blocks to obtain a decomposition of distributions into an infinite series of smooth functions. Of course, we have already such a decomposition at our disposal: $f=\sum_{k} \hat{f}(k) e_{k}^{*}$. But it turns out to be convenient not to consider each Fourier coefficient separately, but to work with projections on dyadic Fourier blocks.

Definition 4. A dyadic partition of unity $(\chi, \rho)$ consists of two nonnegative radial functions $\chi, \rho \in C^{\infty}\left(\mathbb{R}^{d}, \mathbb{R}\right)$, where $\chi$ is supported in a ball $\mathscr{B}=\{|x| \leqslant c\}$ and $\rho$ is supported in an annulus $\mathscr{A}=\{a \leqslant|x| \leqslant b\}$ for suitable $a, b, c>0$, such that

$$
\text { 1. } \chi+\sum_{j \geqslant 0} \rho\left(2^{-j} \cdot\right) \equiv 1 \text { and }
$$

2. $\operatorname{supp}(\chi) \cap \operatorname{supp}\left(\rho\left(2^{-j} \cdot\right)\right)=\emptyset$ for $j \geqslant 1$ and $\operatorname{supp}\left(\rho\left(2^{-i} \cdot\right)\right) \cap$ $\operatorname{supp}\left(\rho\left(2^{-j} \cdot\right)\right)=\emptyset$ for all $i, j \geqslant 0$ with $|i-j| \geqslant 1$.

We will often write $\rho_{-1}=\chi$ and $\rho_{j}=\rho\left(2^{-j}\right.$.) for $j \geqslant 0$.

Dyadic partitions of unity exist, see [1]. From now on we fix a dyadic partition of unity $(\chi, \rho)$ and define the dyadic blocks

$$
\Delta_{j} f=\rho_{j}(\mathrm{D}) f=\mathscr{F}^{-1}\left(\rho_{j} \hat{f}\right), \quad j \geqslant-1,
$$


where here and in the following we use that every function on $\mathbb{R}^{d}$ can be naturally interpreted as a function on $\mathbb{Z}^{d}$. We also use the notation

$$
S_{j} f=\sum_{i \leqslant j-1} \Delta_{i} f
$$

as well as $K_{i}=(2 \pi)^{d / 2} \mathscr{F}^{-1} \rho_{i}$ so that

$$
K_{i} * f=\mathscr{F}^{-1}\left(\rho_{i} \mathscr{F} f\right)=\Delta_{i} f .
$$

From this representation we can also see the reason for considering smooth partitions rather than indicator functions: From Young's inequality we get only $\left\|\mathbb{I}_{\left[2^{j}, 2^{j+1}\right)}(|\mathrm{D}|) f\right\|_{L^{\infty}} \leq\left\|\mathscr{F}^{-1} \mathbb{I}_{\left[2^{j}, 2^{j+1}\right.}\right\|_{L^{1}}\|f\|_{L^{\infty}} \lesssim j\|f\|_{L^{\infty}}$ for $f \in L^{\infty}$, whereas $\left\|\rho_{j}(\mathrm{D}) f\right\|_{L^{\infty}} \lesssim\|f\|_{L^{\infty}}$ uniformly in $j$.

Every dyadic block has a compactly supported Fourier transform and is therefore in $\mathscr{S}$. It is easy to see that $f=\sum_{j \geqslant-1} \Delta_{j} f=\lim _{j \rightarrow \infty} S_{j} f$ for all $f \in \mathscr{S}^{\prime}$.

For $\alpha \in \mathbb{R}$, the Hölder-Besov space $\mathscr{C}^{\alpha}$ is given by $\mathscr{C}^{\alpha}=B_{\infty, \infty}^{\alpha}\left(\mathbb{T}^{d}, \mathbb{R}\right)$, where for $p, q \in[1, \infty]$ we define

$$
B_{p, q}^{\alpha}=B_{p, q}^{\alpha}\left(\mathbb{T}^{d}, \mathbb{R}\right)=\left\{f \in \mathscr{S}^{\prime}:\|f\|_{B_{p, q}^{\alpha}}=\left(\sum_{j \geqslant-1}\left(2^{j \alpha}\left\|\Delta_{j} f\right\|_{L^{p}}\right)^{q}\right)^{1 / q}<\infty\right\},
$$

with the usual interpretation as $\ell^{\infty}$ norm if $q=\infty$. Then $B_{p, q}^{\alpha}$ is a Banach space and while the norm $\|\cdot\|_{B_{p, q}^{\alpha}}$ depends on $(\chi, \rho)$, the space $B_{p, q}^{\alpha}$ does not and any other dyadic partition of unity corresponds to an equivalent norm (for $(p, q)=(\infty, \infty)$ this follows from Lemma 10 below, for the general case see [1], Lemma 2.69). We write $\|\cdot\|_{\alpha}$ instead of $\|\cdot\|_{B_{\infty, \infty}^{\alpha}}$.

Exercise 9. Let $\delta_{0}$ denote the Dirac delta in 0. Show that $\delta_{0} \in \mathscr{C}^{-d}$.

If $\alpha \in(0, \infty) \backslash \mathbb{N}$, then $\mathscr{C}^{\alpha}$ is the space of $\lfloor\alpha\rfloor$ times differentiable functions whose partial derivatives of order $\lfloor\alpha\rfloor$ are $(\alpha-\lfloor\alpha\rfloor)$-Hölder continuous (see page 99 of [1]). Note however, that for $k \in \mathbb{N}$ the space $\mathscr{C}^{k}$ is strictly larger than $C^{k}$, the space of $k$ times continuously differentiable functions. Below we will give the proof for $\alpha \in(0,1)$, but before we still need some tools.

Recall that Schwartz functions on $\mathbb{R}^{d}$ are functions $f \in C^{\infty}\left(\mathbb{R}^{d}\right)$ such that for every multiindex $\mu$ and all $n \geq 0$ we have

$$
\sup _{x \in \mathbb{R}^{d}}(1+|x|)^{n}\left|\partial^{\mu} f(x)\right|<\infty .
$$

Lemma 6. (Poisson summation) Let $\varphi: \mathbb{R}^{d} \rightarrow \mathbb{C}$ be a Schwartz function. Then

$$
\mathscr{F}^{-1} \varphi(x)=\sum_{k \in \mathbb{Z}^{d}} \mathscr{F}_{\mathbb{R}^{d}}^{-1} \varphi(x+2 \pi k),
$$

for all $x \in \mathbb{T}^{d}$, where $\mathscr{F}_{\mathbb{R}^{d}}^{-1} \varphi(x)=(2 \pi)^{-d / 2} \int_{\mathbb{R}^{d}} \varphi(y) e^{i\langle x, y\rangle} \mathrm{d} y$. 
Proof. Let $g(x)=\sum_{k \in \mathbb{Z}^{d}} \mathscr{F}_{\mathbb{R}^{d}}^{-1} \varphi(x+2 \pi k)$. The function $\mathscr{F}_{\mathbb{R}^{d}}^{-1} \varphi$ is of rapid decay since $\varphi \in \mathscr{S}$ so the sum converges absolutely and defines a continuous function $g: \mathbb{R}^{d} \rightarrow \mathbb{R}$ which is periodic of period $2 \pi$ in every direction. The Fourier transform over the torus $\mathbb{T}^{d}$ of this function is

$$
\begin{aligned}
\mathscr{F} g(y) & =\int_{\mathbb{T}^{d}} e^{-i\langle x, y\rangle} g(x) \frac{\mathrm{d} x}{(2 \pi)^{d / 2}} \\
& =\int_{\mathbb{T}^{d}} \sum_{k \in \mathbb{Z}^{d}} \mathscr{F}_{\mathbb{R}^{d}}^{-1} \varphi(x+2 \pi k) e^{-i\langle x+2 \pi k, y\rangle} \frac{\mathrm{d} x}{(2 \pi)^{d / 2}}
\end{aligned}
$$

since $e^{-i\langle 2 \pi k, y\rangle}=1$ for all $y \in \mathbb{Z}^{d}$. By dominated convergence the sum and the integral can be combined in an overall integration over $\mathbb{R}^{d}$ :

$$
\mathscr{F} g(y)=\int_{\mathbb{R}^{d}} \mathscr{F}_{\mathbb{R}^{d}}^{-1} \varphi(x) e^{-i\langle x, y\rangle} \frac{\mathrm{d} x}{(2 \pi)^{d / 2}}=\mathscr{F}_{\mathbb{R}^{d}} \mathscr{F}_{\mathbb{R}^{d}}^{-1} \varphi(y)=\varphi(y),
$$

where $\mathscr{F}_{\mathbb{R}^{d}} f(x)=\mathscr{F}_{\mathbb{R}^{d}}^{-1} f(-x)$. So we deduce that $g(x)=\mathscr{F}^{-1} \varphi(x)$.

Exercise 10. Show that $\|\cdot\|_{\alpha} \leqslant\|\cdot\|_{\beta}$ for $\alpha \leqslant \beta$, that $\|\cdot\|_{L^{\infty}} \lesssim\|\cdot\|_{\alpha}$ for $\alpha>0$, that $\|\cdot\|_{\alpha} \lesssim\|\cdot\|_{L^{\infty}}$ for $\alpha \leqslant 0$, and that $\left\|S_{j} \cdot\right\|_{L^{\infty}} \lesssim 2^{j \alpha}\|\cdot\|_{\alpha}$ for $\alpha<0$. These inequalities will be very important for us in the following and we will often use them without mentioning it specifically.

Hint: When proving $\|\cdot\|_{\alpha} \lesssim\|\cdot\|_{L^{\infty}}$ for $\alpha \leqslant 0$, you might need Poisson's summation formula.

The following Bernstein inequality is extremely useful when dealing with functions with compactly supported Fourier transform.

Lemma 7. (Bernstein inequality) Let $\mathscr{B}$ be a ball and $k \in \mathbb{N}_{0}$. For any $\lambda \geqslant 1,1 \leqslant p \leqslant q \leqslant \infty$, and $f \in L^{p}$ with $\operatorname{supp}(\mathscr{F} f) \subseteq \lambda \mathscr{B}$ we have

$$
\max _{\mu \in \mathbb{N}^{d}:|\mu|=k}\left\|\partial^{\mu} f\right\|_{L^{q}} \lesssim_{k, \mathscr{B}} \lambda^{k+d\left(\frac{1}{p}-\frac{1}{q}\right)}\|f\|_{L^{p}}
$$

Proof. Let $\psi$ be a compactly supported $C^{\infty}$ function on $\mathbb{R}^{d}$ such that $\psi \equiv 1$ on $\mathscr{B}$ and write $\psi_{\lambda}(x)=\psi\left(\lambda^{-1} x\right)$. Then

$$
\begin{aligned}
\partial^{\mu} f(x) & =\partial^{\mu} \mathscr{F}^{-1}\left(\psi_{\lambda} \mathscr{F} f\right)(x)=(2 \pi)^{d / 2}\left\langle f, \partial^{\mu}\left(\mathscr{F}^{-1} \psi_{\lambda}\right)(x-\cdot)\right\rangle \\
& =(2 \pi)^{d / 2}\left(f * \partial^{\mu}\left(\mathscr{F}^{-1} \psi_{\lambda}\right)\right)(x) .
\end{aligned}
$$

By Young's inequality, we get

$$
\left\|\partial^{\mu} f\right\|_{L^{q}} \lesssim\|f\|_{L^{p}}\left\|\partial^{\mu}\left(\mathscr{F}^{-1} \psi_{\lambda}\right)\right\|_{L^{r}}
$$

where $1+1 / q=1 / p+1 / r$. Now it is a short exercise to verify 


$$
\begin{aligned}
\|\cdot\|_{L^{r}} \leqslant\|\cdot\|_{L^{1}}^{1 / r}\|\cdot\|_{L^{\infty}}^{1-1 / r}, \text { and } & \\
\left\|\partial^{\mu}\left(\mathscr{F}^{-1} \psi_{\lambda}\right)\right\|_{L^{1}} & =\int_{\mathbb{T}^{d}}\left|\sum_{k} \partial^{\mu}\left(\mathscr{F}_{\mathbb{R}^{d}}^{-1} \psi_{\lambda}\right)(x+2 \pi k)\right| \mathrm{d} x \\
& \leqslant \int_{\mathbb{R}^{d}}\left|\partial^{\mu}\left(\mathscr{F}_{\mathbb{R}^{d}}^{-1} \psi_{\lambda}\right)(x)\right| \mathrm{d} x \\
& =\lambda^{|\mu|} \int_{\mathbb{R}^{d}} \lambda^{d}\left|\left(\partial^{\mu} \mathscr{F}_{\mathbb{R}^{d}}^{-1} \psi\right)(\lambda x)\right| \mathrm{d} x \\
& \simeq \lambda^{|\mu|},
\end{aligned}
$$

whereas

$$
\begin{aligned}
\sup _{x \in \mathbb{T}^{d}}\left|\sum_{k} \partial^{\mu}\left(\mathscr{F}_{\mathbb{R}^{d}}^{-1} \psi_{\lambda}\right)(x+2 \pi k)\right| & =\lambda^{d+|\mu|} \sup _{x \in \mathbb{T}^{d}}\left|\sum_{k}\left(\partial^{\mu} \mathscr{F}_{\mathbb{R}^{d}}^{-1} \psi\right)(\lambda(x+2 \pi k))\right| \\
& \lesssim \lambda^{d+|\mu|} \sup _{x \in \mathbb{T}^{d}} \sum_{k}(1+\lambda|x+2 \pi k|)^{-2 d} \\
& \lesssim \lambda^{d+|\mu|} \sup _{x \in \mathbb{T}^{d}} \sum_{k}(1+|x+2 \pi k|)^{-2 d} \\
& \lesssim \lambda^{d+|\mu|}
\end{aligned}
$$

We end up with

$$
\begin{aligned}
\left\|\partial^{\mu} f\right\|_{L^{q}} & \lesssim\|f\|_{L^{p}}\left\|\partial^{\mu}\left(\mathscr{F}^{-1} \psi_{\lambda}\right)\right\|_{L^{r}} \\
& \lesssim\|f\|_{L^{p}} \lambda^{|\mu| / r} \lambda^{(d+|\mu|)(1-1 / r)} \\
& =\|f\|_{L^{p}} \lambda^{|\mu|+d(1 / p-1 / q)} .
\end{aligned}
$$

It then follows immediately that for $\alpha \in \mathbb{R}, f \in \mathscr{C}^{\alpha}, \mu \in \mathbb{N}_{0}^{d}$, we have $\partial^{\mu} f \in \mathscr{C}^{\alpha-|\mu|}$. Another simple application of the Bernstein inequalities is the Besov embedding theorem, the proof of which we leave as an exercise.

Lemma 8. (Besov embedding) Let $1 \leqslant p_{1} \leqslant p_{2} \leqslant \infty$ and $1 \leqslant q_{1} \leqslant$ $q_{2} \leqslant \infty$, and let $\alpha \in \mathbb{R}$. Then $B_{p_{1}, q_{1}}^{\alpha}$ is continuously embedded into $B_{p_{2}, q_{2}}^{\alpha-d\left(1 / p_{1}-1 / p_{2}\right)}$.

Exercise 11. In the setting of Exercise 2, use Besov embedding to show that $\mathbb{E}\left[\|\tilde{\xi}\|_{-d / 2-\varepsilon}^{p}\right]<\infty$ for all $p \geqslant 1$ and $\varepsilon>0$ (in particular $\tilde{\xi} \in \mathscr{C}^{-d / 2-}$ almost surely).

Hint: Estimate $\mathbb{E}\left[\|\tilde{\xi}\|_{B_{2 p, 2 p}^{\alpha}}^{2 p}\right]$ using Gaussian hypercontractivity (equivalence of moments).

As another application of the Bernstein inequality, let us show that $\mathscr{C}^{\alpha}=C^{\alpha}$ for $\alpha \in(0,1)$. 
Lemma 9. For $\alpha \in(0,1)$ we have $\mathscr{C}^{\alpha}=C^{\alpha}$, the space of $\alpha$-Hölder continuous functions, and

$$
\|f\|_{\alpha} \simeq\|f\|_{C^{\alpha}}=\|f\|_{L^{\infty}}+\sup _{x \neq y} \frac{|f(x)-f(y)|}{d_{\mathbb{T}^{d}}(x, y)^{\alpha}},
$$

where $d_{\mathbb{T}^{d}}(x, y)$ denotes the canonical distance on $\mathbb{T}^{d}$.

Proof. Start by noting that for $f \in \mathscr{C}^{\alpha}$ we have $\|f\|_{L^{\infty}} \leqslant \sum_{j}\left\|\Delta_{j} f\right\|_{L^{\infty}} \leqslant$ $\sum_{j} 2^{-j \alpha}\|f\|_{\alpha} \lesssim\|f\|_{\alpha}$. Let now $x \neq y \in \mathbb{T}^{d}$ and choose $j_{0}$ with $2^{-j_{0}} \simeq d_{\mathbb{T}^{d}}(x, y)$. For $j \leqslant j_{0}$ we use Bernstein's inequality to obtain

$$
\begin{aligned}
\left|\Delta_{j} f(x)-\Delta_{j} f(y)\right| & \lesssim\left\|\mathrm{D} \Delta_{j} f\right\|_{L^{\infty}} d_{\mathbb{T}^{d}}(x, y) \\
& \lesssim 2^{j}\left\|\Delta_{j} f\right\|_{L^{\infty}} d_{\mathbb{T}^{d}}(x, y) \\
& \leqslant 2^{j(1-\alpha)}\|f\|_{\alpha} d_{\mathbb{T}^{d}}(x, y),
\end{aligned}
$$

whereas for $j>j_{0}$ we simply estimate

$$
\left|\Delta_{j} f(x)-\Delta_{j} f(y)\right| \lesssim\left\|\Delta_{j} f\right\|_{L^{\infty}} \lesssim 2^{-j \alpha}\|f\|_{\alpha} .
$$

Summing over $j$, we get

$$
\begin{aligned}
|f(x)-f(y)| & \leqslant \sum_{j \leqslant j_{0}} 2^{j(1-\alpha)}\|f\|_{\alpha} d_{\mathbb{T}^{d}}(x, y)+\sum_{j>j_{0}} 2^{-j \alpha}\|f\|_{\alpha} \\
& \simeq\|f\|_{\alpha}\left(2^{j_{0}(1-\alpha)} d_{\mathbb{T}^{d}}(x, y)+2^{-j_{0} \alpha}\right) \simeq\|f\|_{\alpha} d_{\mathbb{T}^{d}}(x, y)^{\alpha} .
\end{aligned}
$$

Conversely, if $f \in C^{\alpha}$, then we estimate $\left\|\Delta_{-1} f\right\|_{L^{\infty}} \lesssim\|f\|_{L^{\infty}}$. For $j \geqslant 0$, the function $\rho_{j}$ satisfies $\int\left(\mathscr{F}^{-1} \rho_{j}\right)(x) \mathrm{d} x=0$, and therefore

$$
\begin{aligned}
\left|\Delta_{j} f(x)\right| & =\left|\int_{\mathbb{T}^{d}} \mathscr{F}^{-1} \rho_{j}(x-y)(f(y)-f(x)) \mathrm{d} y\right| \\
& =\left|\int_{\mathbb{T}^{d}} \sum_{k} \mathscr{F}_{\mathbb{R}^{d}}^{-1} \rho_{j}(x-y+2 \pi k)(f(y)-f(x)) \mathrm{d} y\right| \\
& =\left|\int_{\mathbb{R}^{d}} \mathscr{F}_{\mathbb{R}^{d}}^{-1} \rho_{j}(x-y)(f(y)-f(x)) \mathrm{d} y\right| .
\end{aligned}
$$

Now $|f(y)-f(x)| \leqslant\|f\|_{C^{\alpha}} d_{\mathbb{T}^{d}}(x, y)^{\alpha} \leqslant\|f\|_{C^{\alpha}}|x-y|^{\alpha}$, and thus we end up with

$$
\begin{aligned}
\left|\Delta_{j} f(x)\right| & \leqslant\|f\|_{C^{\alpha}}\left|2^{j d} \int_{\mathbb{R}^{d}}\right|\left(\mathscr{F}_{\mathbb{R}^{d}}^{-1} \rho\right)\left(2^{j}(x-y)\right)|| x-\left.y\right|^{\alpha} \mathrm{d} y \mid \\
& =\|f\|_{C^{\alpha}} 2^{-j \alpha}\left|2^{j d} \int_{\mathbb{R}^{d}}\right|\left(\mathscr{F}_{\mathbb{R}^{d}}^{-1} \rho\right)\left(2^{j}(x-y)\right) \|\left. 2^{j}(x-y)\right|^{\alpha} \mathrm{d} y \mid \\
& \lesssim\|f\|_{C^{\alpha}} 2^{-j \alpha} .
\end{aligned}
$$


The following lemma, a characterization of Besov regularity for functions that can be decomposed into pieces which are localized in Fourier space, will be immensely useful in what follows.

\section{Lemma 10.}

1. Let $\mathscr{A}$ be an annulus, let $\alpha \in \mathbb{R}$, and let $\left(u_{j}\right)$ be a sequence of smooth functions such that $\mathscr{F} u_{j}$ has its support in $2^{j} \mathscr{A}$, and such that $\left\|u_{j}\right\|_{L^{\infty}} \lesssim 2^{-j \alpha}$ for all $j$. Then

$$
u=\sum_{j \geqslant-1} u_{j} \in \mathscr{C}^{\alpha} \quad \text { and } \quad\|u\|_{\alpha} \lesssim \sup _{j \geqslant-1}\left\{2^{j \alpha}\left\|u_{j}\right\|_{L^{\infty}}\right\} .
$$

2. Let $\mathscr{B}$ be a ball, let $\alpha>0$, and let $\left(u_{j}\right)$ be a sequence of smooth functions such that $\mathscr{F} u_{j}$ has its support in $2^{j} \mathscr{B}$, and such that $\left\|u_{j}\right\|_{L^{\infty}} \lesssim 2^{-j \alpha}$ for all $j$. Then

$$
u=\sum_{j \geqslant-1} u_{j} \in \mathscr{C}^{\alpha} \quad \text { and } \quad\|u\|_{\alpha} \lesssim \sup _{j \geqslant-1}\left\{2^{j \alpha}\left\|u_{j}\right\|_{L^{\infty}}\right\} .
$$

Proof. If $\mathscr{F} u_{j}$ is supported in $2^{j} \mathscr{A}$, then $\Delta_{i} u_{j} \neq 0$ only for $i \sim j$. Hence, we obtain

$$
\begin{aligned}
\left\|\Delta_{i} u\right\|_{L^{\infty}} & \leqslant \sum_{j: j \sim i}\left\|\Delta_{i} u_{j}\right\|_{L^{\infty}} \\
& \leqslant \sup _{k \geqslant-1}\left\{2^{k \alpha}\left\|u_{k}\right\|_{L^{\infty}}\right\} \sum_{j: j \sim i} 2^{-j \alpha} \\
& \simeq \sup _{k \geqslant-1}\left\{2^{k \alpha}\left\|u_{k}\right\|_{L^{\infty}}\right\} 2^{-i \alpha} .
\end{aligned}
$$

If $\mathscr{F} u_{j}$ is supported in $2^{j} \mathscr{B}$, then $\Delta_{i} u_{j} \neq 0$ only for $i \lesssim j$. Therefore,

$$
\begin{aligned}
\left\|\Delta_{i} u\right\|_{L^{\infty}} & \leqslant \sum_{j: j \gtrsim i}\left\|\Delta_{i} u_{j}\right\|_{L^{\infty}} \\
& \leqslant \sup _{k \geqslant-1}\left\{2^{k \alpha}\left\|u_{k}\right\|_{L^{\infty}}\right\} \sum_{j: j \gtrsim i} 2^{-j \alpha} \\
& \lesssim \sup _{k \geqslant-1}\left\{2^{k \alpha}\left\|u_{k}\right\|_{L^{\infty}}\right\} 2^{-i \alpha},
\end{aligned}
$$

using $\alpha>0$ in the last step.

When solving SPDEs, we will need the smoothing properties of the heat semigroup. We define $\mathscr{L}^{\alpha}=C \mathscr{C}^{\alpha} \cap C^{\alpha / 2} L^{\infty}$ for $\alpha \in(0,2)$. For $T>0$ we set $\mathscr{L}_{T}^{\alpha}=C_{T} \mathscr{C}^{\alpha} \cap C_{T}^{\alpha / 2} L^{\infty}$ and we equip $\mathscr{L}_{T}^{\alpha}$ with the norm

$$
\|\cdot\|_{\mathscr{L}_{T}^{\alpha}}=\max \left\{\|\cdot\|_{C_{T} \mathscr{C}^{\alpha}},\|\cdot\|_{C_{T}^{\alpha / 2} L^{\infty}}\right\} .
$$


The notation $\mathscr{L}^{\alpha}$ is chosen to be reminiscent of the operator $\mathscr{L}=\partial_{t}-\Delta$ and indeed the parabolic spaces $\mathscr{L}^{\alpha}$ are adapted to $\mathscr{L}$ in the sense that the temporal regularity "counts twice", which is due to the fact that $\mathscr{L}$ contains a first order temporal but a second order spatial derivative. If we would replace $\Delta$ by a fractional Laplacian $-(-\Delta)^{\sigma}$, then we would have to consider the space $C \mathscr{C}^{\alpha} \cap C^{\alpha /(2 \sigma)} L^{\infty}$ instead of $\mathscr{L}^{\alpha}$.

We have the following Schauder estimate on the scale of $\left(\mathscr{L}^{\alpha}\right)_{\alpha}$ spaces:

Lemma 11. Let $\alpha \in(0,2)$ and let $\left(P_{t}\right)_{t \geqslant 0}$ be the semigroup generated by the periodic Laplacian, $\mathscr{F}\left(P_{t} f\right)(k)=e^{-t|k|^{2}} \mathscr{F} f(k)$. For $f \in C \mathscr{C}^{\alpha-2}$ define $J f(t)=\int_{0}^{t} P_{t-s} f_{s} \mathrm{~d} s$. Then $J f$ is the solution to $\mathscr{L} J f=f, J f(0)=0$, and we have

$$
\|J f\|_{\mathscr{L}_{T}^{\alpha}} \lesssim(1+T)\|f\|_{C_{T} \mathscr{C}^{\alpha-2}}
$$

for all $T>0$. If $u \in \mathscr{C}^{\alpha}$, then $t \mapsto P_{t} u$ is the solution to $\mathscr{L} P . u=0$, $P_{0} u=u$, and we have

$$
\left\|t \mapsto P_{t} u\right\|_{\mathscr{L}_{T}^{\alpha}} \lesssim\|u\|_{\alpha}
$$

Bibliographic notes. For a gentle introduction to Littlewood-Paley theory and Besov spaces see the recent monograph [1], where most of our results are taken from. There the case of tempered distributions on $\mathbb{R}^{d}$ is considered. The theory on the torus is developed in [31]. The Schauder estimates for the heat semigroup are classical and can be found in $[14,16]$. 


\section{Chapter 4}

\section{Diffusion in a random environment}

Let us consider the following $d$-dimensional homogenization problem. Fix $\varepsilon>0$ and let $u^{\varepsilon}: \mathbb{R}_{+} \times \mathbb{T}^{d} \rightarrow \mathbb{R}$ be the solution to the Cauchy problem

$$
\partial_{t} u^{\varepsilon}(t, x)=\Delta u^{\varepsilon}(t, x)+\varepsilon^{-\alpha} V(x / \varepsilon) u^{\varepsilon}(t, x), \quad u^{\varepsilon}(0)=u_{0},
$$

where $V: \mathbb{T}_{\varepsilon}^{d} \rightarrow \mathbb{R}$ is a random field defined on the rescaled torus $\mathbb{T}_{\varepsilon}^{d}=\left(\mathbb{R} /\left(2 \pi \varepsilon^{-1} \mathbb{Z}\right)\right)^{d}$. This model describes the diffusion of particles in a random medium (replacing $\partial_{t}$ by $i \partial_{t}$ gives the Schrödinger equation of a quantum particle evolving in a random potential). For a review of related results the reader can give a look at the recent paper of $\mathrm{Bal}$ and $\mathrm{Gu}[2]$. The limit $\varepsilon \rightarrow 0$ corresponds to looking at the large scale behavior of the model since (4.1) can be understood as the equation for the macroscopic density $u^{\varepsilon}(t, x)=u\left(t / \varepsilon^{2}, x / \varepsilon\right)$ which corresponds to a microscopic density $u: \mathbb{R}_{+} \times \mathbb{T}_{\varepsilon}^{d} \rightarrow \mathbb{R}$ evolving according to the parabolic equation

$$
\partial_{t} u(t, x)=\Delta u(t, x)+\varepsilon^{2-\alpha} V(x) u(t, x), \quad u(0, \cdot)=u_{0}(\varepsilon \cdot) .
$$

Slightly abusing notation, we do not index $u$ or $V$ by $\varepsilon$ despite the fact that they of course depend on it. We assume that $V: \mathbb{T}_{\varepsilon}^{d} \rightarrow \mathbb{R}$ is Gaussian and has mean zero and homogeneous correlation function $C_{\varepsilon}$ given by

$$
C_{\varepsilon}(x-y)=\mathbb{E}[V(x) V(y)]=(\varepsilon / \sqrt{2 \pi})^{d} \sum_{k \in \varepsilon \mathbb{Z}^{d}} e^{i\langle x-y, k\rangle} R(k) .
$$

On $R: \mathbb{R}^{d} \rightarrow \mathbb{R}_{+}$we make the following hypothesis: for some $\beta \in(0, d]$ we have $R(k)=|k|^{\beta-d} \tilde{R}(k)$ where $\tilde{R} \in \mathscr{S}\left(\mathbb{R}^{d}\right)$ is a smooth radial function of rapid decay. For $\beta<d$ it would be equivalent to require that spatial correlations (in the limit $\varepsilon \rightarrow 0$ ) decay as $|x|^{-\beta}$. For $\beta=d$ this hypothesis 
means that spatial correlations are of rapid decay. Indeed by dominated convergence

$$
\begin{aligned}
\lim _{\varepsilon \rightarrow 0} C_{\varepsilon}(x) & =\int_{\mathbb{R}^{d}} \frac{\mathrm{d} k}{(2 \pi)^{d / 2}} e^{i\langle x, k\rangle} R(k)=\int_{\mathbb{R}^{d}} \frac{\mathrm{d} k}{(2 \pi)^{d / 2}} e^{i\langle x, k\rangle}|k|^{\beta-d} \tilde{R}(k) \\
& =(2 \pi)^{d / 2}\left(\mathscr{F}_{\mathbb{R}^{d}}^{-1}\left(|\cdot|^{\beta-d}\right) * \mathscr{F}_{\mathbb{R}^{d}}^{-1}(\tilde{R})\right)(x) .
\end{aligned}
$$

Here we applied the formula of Exercise 3, which also holds for the Fourier transform on $\mathbb{R}^{d}$. Now $\mathscr{F}_{\mathbb{R}^{d}}^{-1}(\tilde{R}) \in \mathscr{S}\left(\mathbb{R}^{d}\right)$ and $\mathscr{F}_{\mathbb{R}^{d}}^{-1}\left(|\cdot|^{\beta-d}\right)(x) \simeq|x|^{-\beta}$ if $0<\beta<d$ (see for example Proposition 1.29 of [1]), so $\lim _{\varepsilon \rightarrow 0}\left|C_{\varepsilon}(x)\right| \lesssim$ $|x|^{-\beta}$ for $|x| \rightarrow+\infty$.

Let us write $V_{\varepsilon}(x)=\varepsilon^{-\alpha} V(x / \varepsilon)$ so that (4.1) can be rewritten as $\partial_{t} u^{\varepsilon}=\Delta u^{\varepsilon}+V_{\varepsilon} u^{\varepsilon}$, and let us compute the variance of the LittlewoodPaley blocks of $V_{\varepsilon}$.

In order to perform more easily some computations we can introduce a family of centered complex Gaussian random variables $\{g(k)\}_{k \in \varepsilon \mathbb{Z}_{0}}$ such that $g(k)^{*}=g(-k)$ and $\mathbb{E}\left[g(k) g\left(k^{\prime}\right)\right]=\delta_{k+k^{\prime}=0}$ and represent $V_{\varepsilon}(x)$ as

$$
V_{\varepsilon}(x)=\frac{\varepsilon^{d / 2-\alpha}}{(\sqrt{2 \pi})^{d / 2}} \sum_{k \in \varepsilon \mathbb{Z}^{d}} e^{i\langle x, k / \varepsilon\rangle} \sqrt{R(k)} g(k) .
$$

Lemma 12. Assume $\beta-2 \alpha \geqslant 0$. We have for any $\varepsilon>0$ and $i \geqslant 0$ and any $0 \leqslant \kappa \leqslant \beta-2 \alpha$ :

$$
\mathbb{E}\left[\left|\Delta_{i} V_{\varepsilon}(x)\right|^{2}\right] \lesssim 2^{(2 \alpha+\kappa) i} \varepsilon^{\kappa}
$$

This estimate implies that if $\beta>2 \alpha$, then for all $\delta>0$ we have $V_{\varepsilon} \rightarrow 0$ in $L^{2}\left(\Omega ; B_{2,2}^{-\alpha-\delta}\left(\mathbb{T}^{d}\right)\right)$ as $\varepsilon \rightarrow 0$.

Proof. A spectral computation gives

$$
\Delta_{i} V_{\varepsilon}(x)=\frac{\varepsilon^{d / 2-\alpha}}{(\sqrt{2 \pi})^{d / 2}} \sum_{k \in \varepsilon \mathbb{Z}^{d}} e^{i\langle x, k / \varepsilon\rangle} \rho_{i}(k / \varepsilon) \sqrt{R(k)} g(k)
$$

so

$$
\begin{aligned}
\mathbb{E}\left[\left|\Delta_{i} V_{\varepsilon}(x)\right|^{2}\right] & =\varepsilon^{d}(\sqrt{2 \pi})^{-d} \varepsilon^{-2 \alpha} \sum_{k \in \varepsilon \mathbb{Z}^{d}} \rho_{i}(k / \varepsilon)^{2} R(k) \\
& =(\sqrt{2 \pi})^{-d} \varepsilon^{d-2 \alpha} \sum_{k \in \varepsilon \mathbb{Z}^{d}} \rho\left(k /\left(\varepsilon 2^{i}\right)\right)^{2} R(k) \\
& \lesssim \varepsilon^{d-2 \alpha} 2^{i d} \sup _{k \in \varepsilon 2^{i} \mathscr{A}} R(k),
\end{aligned}
$$

where $\mathscr{A}$ is the annulus in which $\rho$ is supported. Now recall that $\beta \leq d$ so that $\left(\varepsilon 2^{i}\right)^{\beta-d} \geq 1$ whenever $\varepsilon 2^{i} \leqslant 1$, which leads to $\mathbb{E}\left[\left|\Delta_{i} V_{\varepsilon}(x)\right|^{2}\right] \lesssim$ $2^{i d} \varepsilon^{d-2 \alpha}\left(\varepsilon 2^{i}\right)^{\beta-d}=\varepsilon^{\beta-2 \alpha} 2^{i \beta}$ in that case. The assumption $\beta-2 \alpha \geqslant 0$ 
then implies $\mathbb{E}\left[\left|\Delta_{i} V_{\varepsilon}(x)\right|^{2}\right] \lesssim 2^{(2 \alpha+\kappa) i} \varepsilon^{\kappa}$ for any $0 \leqslant \kappa \leqslant \beta-2 \alpha$. In the case $\varepsilon 2^{i}>1$ we use that $\int_{\mathbb{R}^{d}} R(k) \mathrm{d} k<+\infty$ to estimate

$$
\varepsilon^{d} \sum_{k \in \varepsilon \mathbb{Z}^{d}} \rho\left(k /\left(\varepsilon 2^{i}\right)\right)^{2} R(k) \leqslant \varepsilon^{d} \sum_{k \in \mathbb{Z}^{d}} R(\varepsilon k) \lesssim \int_{\mathbb{R}^{d}} R(k) \mathrm{d} k<+\infty,
$$

and then $\mathbb{E}\left[\left|\Delta_{i} V_{\varepsilon}(x)\right|^{2}\right] \lesssim \varepsilon^{-2 \alpha} \lesssim 2^{2 \alpha i}\left(\varepsilon 2^{i}\right)^{\kappa}$ for any small $\kappa>0$.

Remark 4. Using Gaussian hypercontractivity, we get from Lemma 12 that

$$
\mathbb{E}\left[\left|\Delta_{i} V_{\varepsilon}(x)\right|^{2 p}\right] \lesssim \mathbb{E}\left[\left|\Delta_{i} V_{\varepsilon}(x)\right|^{2}\right]^{p} \lesssim 2^{(2 \alpha+\kappa) p i} \varepsilon^{\kappa p}
$$

whenever $p \geq 1$, and therefore

$$
\lim _{\varepsilon \rightarrow 0} \mathbb{E}\left[\left\|V_{\varepsilon}\right\|_{B_{2 p, 2 p}^{-\alpha-\delta}}^{2 p}\right]=\lim _{\varepsilon \rightarrow 0} \sum_{i \geq-1} 2^{i(-\alpha-\delta) 2 p} \int_{\mathbb{T}} \mathbb{E}\left[\left|\Delta_{i} V_{\varepsilon}(x)\right|^{2 p}\right] \mathrm{d} x=0
$$

whenever $\delta>0$. By the Besov embedding theorem, this shows that for all $p, \delta>0$

$$
\lim _{\varepsilon \rightarrow 0} \mathbb{E}\left[\left\|V_{\varepsilon}\right\|_{\mathscr{C}-\alpha-\delta}^{p}\right]=0 .
$$

Slightly improving the computation carried out in equation (4.2) we can also see that if $\beta-2 \alpha<0$, then essentially $V_{\varepsilon}$ does not converge in any reasonable sense since the variance of the Littlewood-Paley blocks explodes.

Remark 5. The same calculation as in (4.2) shows that

$$
\mathbb{E}\left[\Delta_{i} V_{\varepsilon}(x) \Delta_{j} V_{\varepsilon}(x)\right]=0
$$

whenever $|i-j|>1$, because in that case $\rho_{i} \rho_{j} \equiv 0$.

The previous analysis shows that it is reasonable to take $\alpha \leqslant \beta / 2$ in order to have some hope of obtaining a well defined limit as $\varepsilon \rightarrow 0$. In this case $V_{\varepsilon}$ stays bounded in probability (at least) in spaces of distributions of regularity $-\alpha-$. This brings us to the problem of obtaining estimates for the parabolic PDE

$$
\mathscr{L} u^{\varepsilon}(t, x)=\left(\partial_{t}-\Delta\right) u^{\varepsilon}(t, x)=V_{\varepsilon}(x) u^{\varepsilon}(t, x), \quad(t, x) \in[0, T] \times \mathbb{T}^{d},
$$

depending only on negative regularity norms of $V_{\varepsilon}$. On one side the regularity of $u^{\varepsilon}$ is then limited by the regularity of the right hand side which cannot be better than that of $V_{\varepsilon}$. On the other side the product of $V_{\varepsilon}$ with $u^{\varepsilon}$ can cause problems since we try to multiply an (a priori) irregular object with one of limited regularity.

Assume that $V_{\varepsilon}$ converges to zero in $\mathscr{C}^{\gamma-2}$ for $\gamma>0$. It is then reasonable to assume that also $V_{\varepsilon} u^{\varepsilon} \in C_{T} \mathscr{C}^{\gamma-2}$, uniformly in $\varepsilon>0$, and 
that $u^{\varepsilon} \in C_{T} \mathscr{C}^{\gamma}$ as a consequence of the regularizing effect of the heat operator (Lemma 11). We will see in Section 5.1 below that the product $V_{\varepsilon} u^{\varepsilon}$ is under control only if $\gamma+\gamma-2>0$, that is if $\gamma>1$. If $V_{\varepsilon} \rightarrow 0$ in $\mathscr{C}^{-1+}$, it is not difficult to show that $u^{\varepsilon}$ converges as $\varepsilon \rightarrow 0$ to the solution $u$ of the linear equation $\mathscr{L} u=0$ (for example this will follow from our analysis below, but in fact it is much simpler to show). In this case the random potential will not have any effect in the limit.

The interesting situation then is when $\gamma \leqslant 1$. To understand what could happen in this case let us use a simple transformation of the solution. Write $u^{\varepsilon}=\exp \left(X^{\varepsilon}\right) v^{\varepsilon}$ where $X^{\varepsilon}$ satisfies the equation $\mathscr{L} X^{\varepsilon}=V_{\varepsilon}$ with initial condition $X^{\varepsilon}(0, \cdot)=0$. Then

$$
\begin{aligned}
\mathscr{L} u^{\varepsilon} & =\exp \left(X^{\varepsilon}\right)\left(v^{\varepsilon} \mathscr{L} X^{\varepsilon}+\mathscr{L} v^{\varepsilon}-v^{\varepsilon}\left(\partial_{x} X^{\varepsilon}\right)^{2}-2\left\langle\partial_{x} X^{\varepsilon}, \partial_{x} v^{\varepsilon}\right\rangle_{\mathbb{R}^{d}}\right) \\
& =\exp \left(X^{\varepsilon}\right) v^{\varepsilon} V_{\varepsilon} .
\end{aligned}
$$

Since $\exp \left(X^{\varepsilon}\right)>0$ on $[0, T] \times \mathbb{T}^{d}$, this implies that $v^{\varepsilon}$ satisfies

$$
\mathscr{L} v^{\varepsilon}-v^{\varepsilon}\left|\partial_{x} X^{\varepsilon}\right|^{2}-2\left\langle\partial_{x} X^{\varepsilon}, \partial_{x} v^{\varepsilon}\right\rangle_{\mathbb{R}^{d}}=0, \quad(t, x) \in[0, T] \times \mathbb{T}^{d} .
$$

Our Schauder estimates imply that $X^{\varepsilon}=J V_{\varepsilon} \in C_{T} \mathscr{C}^{\gamma}$ with uniform bounds in $\varepsilon>0$, so that the problematic term is $\left|\partial_{x} X^{\varepsilon}\right|^{2}$ for which this estimate does not guarantee existence.

Note that $J\left(e^{i\langle\cdot, k\rangle}\right)(t, x)=e^{i\langle x, k\rangle}\left(1-e^{-t|k|^{2}}\right) /|k|^{2}$, which yields

$$
\partial_{x} X^{\varepsilon}(t, x)=\frac{\varepsilon^{d / 2-\alpha}}{(\sqrt{2 \pi})^{d / 2}} \sum_{k \in \varepsilon \mathbb{Z}_{0}^{d}} e^{i\langle x, k / \varepsilon\rangle} G_{\varepsilon}(t, k) g(k)
$$

where $\mathbb{Z}_{0}^{d}=\mathbb{Z}^{d} \backslash\{0\}$ and where

$$
G_{\varepsilon}(t, k)=i \frac{k}{\varepsilon} \frac{\left[1-e^{-t|k / \varepsilon|^{2}}\right]}{|k / \varepsilon|^{2}} \sqrt{R(k)} .
$$

Lemma 13. Assume that

$$
\sigma^{2}=(\sqrt{2 \pi})^{d} \int_{\mathbb{R}^{d}} \frac{R(k)}{k^{2}} \mathrm{~d} k<+\infty .
$$

Then if $\alpha=1$ and $t>0$ we have

$$
\lim _{\varepsilon \rightarrow 0} \mathbb{E}\left[\left|\partial_{x} X^{\varepsilon}\right|^{2}(t, x)\right]=\sigma^{2}
$$

and if $\alpha<1$ and $t>0$

$$
\lim _{\varepsilon \rightarrow 0} \mathbb{E}\left[\left(\left|\partial_{x} X^{\varepsilon}\right|\right)^{2}(t, x)\right]=0 .
$$

Moreover

$$
\operatorname{Var}\left[\Delta_{q}\left(\left|\partial_{x} X^{\varepsilon}\right|^{2}\right)(t, x)\right] \lesssim \varepsilon^{4-4 \alpha} \min \left(\sigma^{4},\left(\varepsilon 2^{q}\right)^{\beta-2}\|\tilde{R}\|_{\infty} \sigma^{2}\right) .
$$


Proof. A computation similar to that leading to equation (4.2) gives

$$
\begin{aligned}
\mathbb{E}\left[\left|\partial_{x} X^{\varepsilon}\right|^{2}(t, x)\right] & =\varepsilon^{d}(\sqrt{2 \pi})^{d} \varepsilon^{-2 \alpha} \sum_{k \in \varepsilon \mathbb{Z}_{0}^{d}}|k / \varepsilon|^{2}\left[\int_{0}^{t} e^{-(t-s)|k / \varepsilon|^{2}} \mathrm{~d} s\right]^{2} R(k) \\
& =\varepsilon^{d}(\sqrt{2 \pi})^{d} \varepsilon^{2-2 \alpha} \sum_{k \in \varepsilon \mathbb{Z}_{0}^{d}} \frac{\left[1-e^{-t(k / \varepsilon)^{2}}\right]^{2}}{k^{2}} R(k),
\end{aligned}
$$

which for $\varepsilon \rightarrow 0, t>0$, and $\alpha \leq 1$ tends to

$$
\lim _{\varepsilon \rightarrow 0} \mathbb{E}\left[\left|\partial_{x} X^{\varepsilon}\right|^{2}(t, x)\right]=\mathbb{I}_{\alpha=1}(\sqrt{2 \pi})^{d} \int_{\mathbb{R}^{d}} \frac{R(k)}{k^{2}} \mathrm{~d} k=\mathbb{I}_{\alpha=1} \sigma^{2}
$$

Let us now study the variance of $\left|\partial_{x} X^{\varepsilon}\right|^{2}(t, x)$. Using equation (4.3) we have

$$
\begin{aligned}
& \Delta_{q}\left(\left|\partial_{x} X^{\varepsilon}\right|^{2}\right)(t, x) \\
= & \frac{\varepsilon^{d-2 \alpha}}{(2 \pi)^{d / 2}} \sum_{k_{1}, k_{2} \in \varepsilon \mathbb{Z}_{0}^{d}} e^{i\left\langle k_{1}+k_{2}, x / \varepsilon\right\rangle} \rho_{q}\left(\left(k_{1}+k_{2}\right) / \varepsilon\right) G_{\varepsilon}\left(t, k_{1}\right) G_{\varepsilon}\left(t, k_{2}\right) g\left(k_{1}\right) g\left(k_{2}\right) .
\end{aligned}
$$

By Wick's theorem ([22], Theorem 1.28)

$$
\begin{aligned}
\operatorname{Cov}\left(g\left(k_{1}\right) g\left(k_{2}\right), g\left(k_{1}^{\prime}\right) g\left(k_{2}^{\prime}\right)\right)= & \mathbb{E}\left[g\left(k_{1}\right) g\left(k_{1}^{\prime}\right)\right] \mathbb{E}\left[g\left(k_{2}\right) g\left(k_{2}^{\prime}\right)\right] \\
& +\mathbb{E}\left[g\left(k_{1}\right) g\left(k_{2}^{\prime}\right)\right] \mathbb{E}\left[g\left(k_{2}\right) g\left(k_{1}^{\prime}\right)\right] \\
= & \mathbb{I}_{k_{1}+k_{1}^{\prime}=k_{2}+k_{2}^{\prime}=0}+\mathbb{I}_{k_{1}+k_{2}^{\prime}=k_{2}+k_{1}^{\prime}=0},
\end{aligned}
$$

which implies

$\operatorname{Var}\left[\Delta_{q}\left(\left|\partial_{x} X^{\varepsilon}\right|^{2}\right)(t, x)\right]=2 \frac{\varepsilon^{2 d-4 \alpha}}{(2 \pi)^{d}} \sum_{k_{1}, k_{2} \in \varepsilon \mathbb{Z}_{0}^{d}}\left(\rho_{q}\left(\left(k_{1}+k_{2}\right) / \varepsilon\right)\right)^{2}\left|G_{\varepsilon}\left(t, k_{1}\right)\right|^{2}\left|G_{\varepsilon}\left(t, k_{2}\right)\right|^{2}$.

For any $q \geqslant 0$ (the case $q=-1$ is left to the reader), the variables $k_{1}$ and $k_{2}$ are bounded away from 0 and we have

$$
\operatorname{Var}\left[\Delta_{q}\left(\left|\partial_{x} X^{\varepsilon}\right|^{2}\right)(t, x)\right] \lesssim \varepsilon^{2 d+4-4 \alpha} \sum_{k_{1}, k_{2} \in \varepsilon \mathbb{Z}_{0}^{d}}\left(\rho_{q}\left(\left(k_{1}+k_{2}\right) / \varepsilon\right)\right)^{2} \frac{\left|R\left(k_{1}\right)\right|\left|R\left(k_{2}\right)\right|}{\left|k_{1}\right|^{2}\left|k_{2}\right|^{2}}
$$

A first estimate is obtained by just dropping the factor $\rho_{q}\left(\left(k_{1}+k_{2}\right) / \varepsilon\right)$ and results in the bound

$$
\operatorname{Var}\left[\Delta_{q}\left(\left|\partial_{x} X^{\varepsilon}\right|^{2}\right)(t, x)\right] \lesssim \varepsilon^{2 d+4-4 \alpha} \sum_{k_{1}, k_{2} \in \varepsilon \mathbb{Z}_{0}^{d}} \frac{\left|R\left(k_{1}\right)\right|\left|R\left(k_{2}\right)\right|}{\left|k_{1}\right|^{2}\left|k_{2}\right|^{2}} \lesssim \varepsilon^{4-4 \alpha} \sigma^{4}
$$


Another estimate proceeds by taking into account the constraint given by the support of $\rho_{q}\left(\left(k_{1}+k_{2}\right) / \varepsilon\right)$. In order to satisfy $k_{1}+k_{2} \sim \varepsilon 2^{q}$ we must have $k_{2} \lesssim k_{1} \sim \varepsilon 2^{q}$ or $\varepsilon 2^{q} \lesssim k_{1} \sim k_{2}$. In the first case

$$
\begin{aligned}
& \varepsilon^{2 d+4-4 \alpha} \sum_{k_{1}, k_{2} \in \varepsilon \mathbb{Z}_{0}^{d}} \mathbb{I}_{k_{2} \lesssim k_{1} \sim \varepsilon 2^{q}} \frac{\left|R\left(k_{1}\right)\right|\left|R\left(k_{2}\right)\right|}{\left|k_{1}\right|^{2}\left|k_{2}\right|^{2}} \\
& \lesssim 2^{q(\beta-2)} \varepsilon^{d+\beta+2-4 \alpha}\|\tilde{R}\|_{\infty} \sum_{k_{2} \in \varepsilon \mathbb{Z}_{0}^{d}} \mathbb{I}_{k_{2} \lesssim \varepsilon 2^{q}} \frac{\left|R\left(k_{2}\right)\right|}{\left|k_{2}\right|^{2}} \\
& \lesssim\left(\varepsilon 2^{q}\right)^{\beta-2}\|\tilde{R}\|_{\infty} \int \mathrm{d} k \frac{|R(k)|}{|k|^{2}} \\
& \lesssim\left(\varepsilon 2^{q}\right)^{\beta-2} \varepsilon^{4-4 \alpha}\|\tilde{R}\|_{\infty} \sigma^{2}
\end{aligned}
$$

since $\left|R\left(k_{1}\right)\right| /\left|k_{1}\right|^{2} \lesssim\|\tilde{R}\|_{\infty}\left(\varepsilon 2^{q}\right)^{\beta-d-2}$. If $\varepsilon 2^{q} \lesssim k_{1} \sim k_{2}$ we similarly have

$$
\begin{aligned}
& \varepsilon^{2 d+4-4 \alpha} \sum_{k_{1}, k_{2} \in \varepsilon \mathbb{Z}_{0}^{d}} \mathbb{I}_{\varepsilon 2^{q} \lesssim k_{1} \sim k_{2}} \frac{\left|R\left(k_{1}\right)\right|\left|R\left(k_{2}\right)\right|}{\left|k_{1}\right|^{2}\left|k_{2}\right|^{2}} \\
& \lesssim 2^{q(\beta-2)} \varepsilon^{d+\beta+2-4 \alpha}\|\tilde{R}\|_{\infty} \sum_{k_{2} \in \varepsilon \mathbb{Z}_{0}^{d}} \mathbb{I}_{\varepsilon 2^{q} \lesssim k_{2}} \frac{\left|R\left(k_{2}\right)\right|}{\left|k_{2}\right|^{2}} \\
& \lesssim\left(\varepsilon 2^{q}\right)^{\beta-2} \varepsilon^{4-4 \alpha}\|\tilde{R}\|_{\infty} \int \mathrm{d} k \frac{|R(k)|}{|k|^{2}} \\
& \lesssim\left(\varepsilon 2^{q}\right)^{\beta-2} \varepsilon^{4-4 \alpha}\|\tilde{R}\|_{\infty} \sigma^{2} .
\end{aligned}
$$

This lemma shows that the interesting situation is $\alpha=1$. Then, provided $\sigma^{2}<+\infty$ and $\beta>2$ we have $\left|\partial_{x} X^{\varepsilon}\right|^{2}(t) \rightarrow \sigma^{2}$ in $L^{2}\left(\Omega ; \mathscr{C}^{0-}\right)$ for all $t>0$, and in fact the convergence is uniform for $t \in[c, C]$ whenever $0<c<C$. Since all the operations that appear in the equation for $v^{\varepsilon}$ are continuous, it is then easy to see that $v^{\varepsilon}$ converges to the solution of the PDE

$$
\mathscr{L} v=\sigma^{2} v
$$

and since $X^{\varepsilon}$ is a continuous linear functional of $V_{\varepsilon}$, we have $X^{\varepsilon} \rightarrow 0$ in $C_{T} \mathscr{C}^{\gamma}$ and thus we finally obtain the convergence of $\left(u^{\varepsilon}\right)_{\varepsilon>0}$ to the same $v$.

Thus, we have (modulo technical details) shown the following theorem: Theorem 3. Let $\beta \in(0, d]$ and let $R=|\cdot|^{\beta-d} \tilde{R}$, where $\tilde{R} \in \mathscr{S}\left(\mathbb{R}^{d}\right)$ is a smooth radial function of rapid decay, and assume that $\sigma^{2}=$ $(\sqrt{2 \pi})^{d} \int_{\mathbb{R}^{d}} R(k) / k^{2} \mathrm{~d} k<\infty$. Let $V: \mathbb{T}_{\varepsilon}^{d} \rightarrow \mathbb{R}$ be a continuous Gaussian function with mean zero and correlation

$$
\mathbb{E}[V(x) V(y)]=C_{\varepsilon}(x-y)=(\varepsilon / \sqrt{2 \pi})^{d} \sum_{k \in \varepsilon \mathbb{Z}^{d}} e^{i\langle x-y, k\rangle} R(k) .
$$


Consider the solution $u^{\varepsilon}: \mathbb{R}_{+} \times \mathbb{T}^{d} \rightarrow \mathbb{R}$ to the Cauchy problem

$$
\partial_{t} u^{\varepsilon}(t, x)=\Delta u^{\varepsilon}(t, x)+\varepsilon^{-\alpha} V(x / \varepsilon) u^{\varepsilon}(t, x), \quad u^{\varepsilon}(0)=u_{0},
$$

where $u_{0} \in C^{\infty}\left(\mathbb{T}^{d}\right)$. If $\alpha \in(0,1 \wedge \beta / 2)$, then $u^{\varepsilon}$ converges to the solution $u$ of

$$
\partial_{t} u(t, x)=\Delta u(t, x), \quad u(0)=u_{0} .
$$

However, if $1=\alpha<\beta / 2$, then $u^{\varepsilon}$ converges to the solution $v$ of

$$
\partial_{t} v(t, x)=\Delta v(t, x)+\sigma^{2} v(t, x), \quad v(0)=u_{0} .
$$

\subsection{The 2d generalized parabolic Anderson model}

The case $\alpha=1$ and $\beta=2$ remains open in the previous analysis. When $\beta=2$ we cannot expect $\sigma^{2}$ to be finite and moreover from the above computations we see that the variance of $\left|\partial_{x} X^{\varepsilon}\right|^{2}$ remains finite and does not go to zero, so the limiting object should satisfy a stochastic PDE rather than a deterministic one. If we let $\sigma_{\varepsilon}^{2}(t)=\mathbb{E}\left[\left|\partial_{x} X^{\varepsilon}\right|^{2}(t, x)\right]$ (which depends on time but which is easily shown to be independent of $x \in \mathbb{T}^{2}$ ), then we expect that solving the renormalized equation

$$
\mathscr{L} \tilde{u}^{\varepsilon}=V_{\varepsilon} \tilde{u}^{\varepsilon}-\sigma_{\varepsilon}^{2} \tilde{u}^{\varepsilon}
$$

should give rise in the limit to a well defined random field $\tilde{u}$ satisfying $\tilde{u}=e^{X} \tilde{v}$, where

$$
\mathscr{L} \tilde{v}=\tilde{v} \zeta+2\left\langle\partial_{x} X, \partial_{x} \tilde{v}\right\rangle_{\mathbb{R}^{d}}
$$

and where $X$ is the limit of $X^{\varepsilon}$ as $\varepsilon \rightarrow 0$ while $\zeta$ is the limit of $\left(\partial_{x} X^{\varepsilon}\right)^{2}-\sigma_{\varepsilon}^{2}$. The relation of $u^{\varepsilon}$ with $\tilde{u}^{\varepsilon}$ is $\tilde{u}^{\varepsilon}(t, x)=e^{-\int_{0}^{t} \sigma_{\varepsilon}^{2}(s) \mathrm{d} s} u^{\varepsilon}(t, x)$. The renormalization procedure is therefore equivalent to a time-dependent rescaling of the solution to the initial problem. Without renormalization, the solution will simply drift of to $+\infty$, so in order to see a nontrivial behavior, we have to put ourselves in a different reference frame by multiplying with $e^{-\int_{0}^{t} \sigma_{\varepsilon}^{2}(s) \mathrm{d} s}$. One familiar situation where such a need for renormalization arises is in the central limit theorem: If $\left(Y_{n}\right)$ is a sequence of i.i.d. random variables with unit variance and mean $\mu>0$, then $\left(n^{-1 / 2} \sum_{k=1}^{n} Y_{k}\right)$ diverges to $+\infty$, but once we subtract the diverging constants $n^{1 / 2} \mu$ we get that $\left(n^{-1 / 2} \sum_{k=1}^{n} Y_{k}-n^{1 / 2} \mu\right)$ converges weakly to a standard Gaussian distribution.

We will study the renormalization and convergence problem for a more general equation of the form

$$
\mathscr{L} u^{\varepsilon}=F\left(u^{\varepsilon}\right) V_{\varepsilon},
$$


where $F: \mathbb{R} \rightarrow \mathbb{R}$ is a sufficiently smooth function, in general non-linear. One possible motivation is that if $z^{\varepsilon}$ solves the linear $\operatorname{PDE} \mathscr{L} z^{\varepsilon}=z^{\varepsilon} V_{\varepsilon}$ and we set $u^{\varepsilon}=\varphi\left(z^{\varepsilon}\right)$ for some invertible $\varphi: \mathbb{R} \rightarrow \mathbb{R}$ such that $\varphi^{\prime}>0$, then

$\mathscr{L} u^{\varepsilon}=\varphi^{\prime}\left(z^{\varepsilon}\right) \mathscr{L} z^{\varepsilon}-\varphi^{\prime \prime}\left(z^{\varepsilon}\right)\left|\partial_{x} z^{\varepsilon}\right|^{2}=\varphi^{\prime}\left(z^{\varepsilon}\right) z^{\varepsilon} V_{\varepsilon}-\varphi^{\prime \prime}\left(z^{\varepsilon}\right)\left(\varphi^{\prime}\left(z^{\varepsilon}\right)\right)^{-2}\left|\partial_{x} u^{\varepsilon}\right|^{2}$

and thus $u^{\varepsilon}$ satisfies the PDE

$$
\mathscr{L} u^{\varepsilon}=F_{1}\left(u^{\varepsilon}\right) V_{\varepsilon}+F_{2}\left(u^{\varepsilon}\right)\left(\partial_{x} u^{\varepsilon}\right)^{2}
$$

where

$$
F_{1}(x)=\varphi^{\prime}\left(\varphi^{-1}(x)\right) \varphi^{-1}(x) \text { and } F_{2}(x)=-\varphi^{\prime \prime}\left(\varphi^{-1}(x)\right)\left(\varphi^{\prime}\left(\varphi^{-1}(x)\right)\right)^{-2} \text {. }
$$

In the situation we are interested in, the second term in the right hand side is simpler to treat than the first term. So, for the time being, we will drop it and we will concentrate on the equation (4.5) in $d=2$ with $\alpha=1$ and short ranged $(\beta=d)$ potential $V$ which we refer to as generalized parabolic Anderson model (GPAM).

Under these conditions, $V_{\varepsilon}$ converges to the white noise in space which we usually denote with $\xi$ and our aim will be to set up a theory in which the operations involved in the definition of the dynamics of the GPAM are well defined, including the possibility of the renormalization which already appears in the linear case as hinted above.

While the reader should always have in mind a limiting procedure from a well defined model like the ones we were considering so far, in the following we will mostly discuss the limiting equation. The specific phenomena appearing when trying to track the oscillations of the term $F\left(u^{\varepsilon}\right) V_{\varepsilon}$ as $\varepsilon \rightarrow 0$ will be described by a renormalized product $F(u) \diamond \xi$ and so we write the GPAM as

$$
\mathscr{L} u(t, x)=F(u(t, x)) \diamond \xi(x), \quad u(0)=u_{0} .
$$

In the linear case $F(u)=u$, the problem of the renormalization can be solved along the lines suggested above. Another possible line of attack comes from the theory of Gaussian spaces and in particular from Wick products, see for example [21]. However, the definition of the Wick product relies on the concrete chaos expansion of its factors, and since nonlinear functions change the chaos expansion in a complicated way, there is little hope of directly extending the Wick product approach to the nonlinear case and moreover using these non-local (in the probability space) objects can deliver solutions which are not physically acceptable [5].

Equation (4.6) is structurally very similar to the stochastic differential equation

$$
\partial_{t} v(t)=F(v(t)) \partial_{t} B^{H}(t), \quad v(0)=v_{0},
$$


where $B^{H}$ denotes a fractional Brownian motion with Hurst index $H \in$ $(0,1)$. There are many ways to solve $(4.7)$ in the Brownian case. Since we are interested in a way that might extend to (4.6) where the irregularity appears along the two-dimensional spatial variable $x$, we should exclude all approaches based on information, filtrations, and a direction of time; in particular, any approach that works for $H \neq 1 / 2$ might seem promising. But Lyons' theory of rough paths [25] equips us exactly with the techniques we need to solve (4.7) for general $H$. More precisely, if for $H>1 / 3$ we are given $\int_{0}^{\cdot} B_{s}^{H} \mathrm{~d} B_{s}^{H}$, then we can use the controlled rough path integral [12] to make sense of $\int_{0}^{\cdot} f_{s} \mathrm{~d} B_{s}^{H}$ for any $f$ which "looks like" $B^{H}$, and this allows us to solve (4.7). So the main ingredients required for controlled rough paths are the integral $\int_{0}^{\cdot} B_{s}^{H} \mathrm{~d} B_{s}^{H}$ for the reference path $B^{H}$, and the fact that we can describe paths which look like $B^{H}$. It is worthwhile to note that while we need probability theory to construct $\int_{0}^{\cdot} B_{s}^{H} \mathrm{~d} B_{s}^{H}$, the construction of $\int_{0}^{\cdot} f_{s} \mathrm{~d} B_{s}^{H}$ is achieved using pathwise arguments and it is given as a continuous map of $f$ and $\left(B^{H}, \int_{0}^{\cdot} B_{s}^{H} \mathrm{~d} B_{s}^{H}\right)$. As a consequence, the solution to the SDE (4.7) depends pathwise continuously on $\left(B^{H}, \int_{0}^{\cdot} B_{s}^{H} \mathrm{~d} B^{H}\right)$.

By the structural similarity of (4.6) and (4.7), we might hope to extend the rough path approach to (4.6). The equivalent of $B^{H}$ is given by the solution $\vartheta$ to $\mathscr{L} \vartheta=\xi, \vartheta(0)=0$, and the equivalent of $\int_{0}^{\cdot} B_{s}^{H} \mathrm{~d} B_{s}^{H}$ turns out to be the renormalized product $\vartheta \diamond \xi$. Then we might hope that given $\vartheta \diamond \xi$ we are able to define $f \diamond \xi$ for all $f$ that "look like $\vartheta$ ", however this is to be interpreted. Of course, rough paths can only be applied to functions of a one-dimensional index variable, while for (4.6) the problem lies in the irregularity of $\xi$ in the spatial variable $x \in \mathbb{T}^{2}$.

In the following we combine the ideas from controlled rough paths with Bony's paraproduct, a tool from functional analysis that allows us to extend rough paths to functions of a multidimensional parameter. Using the paraproduct, we are able to make precise in a simple way what we mean by "distributions looking like a reference distribution". We can then define products of suitable distributions and solve (4.6) as well as many other interesting singular SPDEs.

\subsection{More singular problems}

Keeping the homogenization problem as leitmotiv for these lectures, we could consider also space-time varying environments $V_{\varepsilon}(t, x)=$ $\varepsilon^{-\alpha} V\left(t / \varepsilon^{2}, x / \varepsilon\right)$. The scaling of the temporal variable is chosen so that it is compatible with the diffusive scaling from a microscopic description, where $V(t, x)$ has typical variation in space and time in scales of order 1. Assume that $d=1$, then when the random field $V$ is Gaussian, zero mean, and with short-range space-time correlations, the natural choice for the magnitude of the macroscopic fluctuations is $\alpha=3 / 2$. In this 
case $V_{\varepsilon}$ converges as $\varepsilon \rightarrow 0$ to a space-time white noise $\xi$. Understanding the limit dynamics as $\varepsilon \rightarrow 0$ of the solution $u^{\varepsilon}$ to the linear equation $\mathscr{L} u^{\varepsilon}=V_{\varepsilon} u^{\varepsilon}$ represents now a more difficult problem than in the time independent situation. A Gaussian computation shows that the random field $X^{\varepsilon}$, solution to $\mathscr{L} X^{\varepsilon}=V_{\varepsilon}$ (e.g. with zero initial condition), stays bounded in $C_{T} \mathscr{C}^{1 / 2-}$ as $\varepsilon \rightarrow 0$. Since $\mathscr{L}$ is a second order operator (if we use an appropriate parabolic weighting of the time and space regularities), $\xi$ is expected to live in a space of distributions of regularity $-3 / 2-$. This is to be compared with the -1 - of the space white noise which had to be dealt with in the GPAM. Renormalization effects are then expected to be stronger in this setting and the limiting object, which we denote with $w$, should satisfy a (suitably renormalized) linear stochastic heat equation with multiplicative noise (SHE)

$$
\mathscr{L} w(t, x)=w(t, x) \diamond \xi(t, x), \quad w(0)=w_{0} .
$$

As indicated by the computations in the more regular case, it is useful to consider the change of variables $w=e^{h}$ which is called Cole-Hopf transformation. Here $h:[0, \infty) \times \mathbb{T} \rightarrow \mathbb{R}$ is a new unknown which satisfies now the Kardar-Parisi-Zhang (KPZ) equation:

$$
\mathscr{L} h(t, x)=\left(\partial_{x} h(t, x)\right)^{\diamond}+\xi(t, x), \quad h(0)=h_{0}
$$

where the difficulty comes now from the squaring of the derivative but which has the nice feature to be additively perturbed by the space-time white noise, a feature which simplifies many considerations. Another relevant model in applications is obtained by taking the space derivative of KPZ and letting $u(t, x)=\partial_{x} h(t, x)$ in order to obtain the stochastic conservation law

$$
\mathscr{L} u(t, x)=\partial_{x}(u(t, x))^{\diamond}+\partial_{x} \xi(t, x), \quad u(0)=u_{0},
$$

which we will refer to as the stochastic Burgers equation (SBE). In all these cases, $\diamond$ denotes a suitably renormalized product.

The KPZ equation was derived by Kardar-Parisi-Zhang in 1986 as a universal model for the random growth of an interface [24]. For a long time it could not be solved due to the fact that there was no way to make sense of the nonlinearity $\left(\partial_{x} h\right)^{\diamond 2}$ in (4.9). The only way to make sense of KPZ was to apply the Cole-Hopf transform [3]: solve SHE (4.8) (which is accessible to Itô integration) and set $h=\log w$. But there was no intrinsic interpretation of what it means to solve (4.9). Finally, in 2011 Hairer [18] used rough paths to give a meaning to the equation and to obtain solutions directly at the KPZ level. In Section 6 we will sketch how to recover his solution in the paracontrolled setting. Applications of the techniques used by Hairer to solve the KPZ problem to a more general homogenization problem with ergodic potentials (not necessarily Gaussian) have been studied in [20]. 


\subsection{Hairer's regularity structures}

In [19], Hairer introduces a theory of regularity structures which can also be considered a generalization of the theory of controlled rough paths to functions of a multidimensional index variable. Hairer fundamentally rethinks the notion of regularity. Usually a function is called smooth if it can be approximated around every point by a polynomial of a given degree (the Taylor polynomial). Naturally, the solution to an SPDE driven by -say- Gaussian space-time white noise is not smooth in that sense. So in Hairer's theory, a function is called smooth if locally it can be approximated by the noise (and higher order terms constructed from the noise). This induces a natural topology in which the solutions to semilinear SPDEs depend continuously on the driving signal.

At this date it seems that the theory of regularity structures has a wider range of applicability than the paracontrolled approach described in [14], but also at the expense of a very deep conceptual sophistication. There are problems (like the one-dimensional heat equation with multiplicative noise and general nonlinearity) that cannot be solved using paracontrolled distributions, but these problems seem also quite difficult (even if doable and there is work in progress) to tackle with regularity structures. Moreover, equations of a more general kind, say dispersive equations or wave equations, are still poorly (or not at all) understood in both approaches. 


\section{Chapter 5}

\section{The paracontrolled PAM}

As we have tried to motivate in the previous sections we are looking for a theory for PAM which describes the possible limits of the equation

$$
\mathscr{L} u=F(u) \eta
$$

driven by sufficiently regular $\eta$ but as $\eta$ is converging to the space white noise $\xi$. From this point of view we are looking for a priori estimates on the solution $u$ to (5.1) which depend only on distributional norms of $\eta$. So in the following we will assume that we have at hand only a uniform control of $\eta$ in $C_{T} \mathscr{C}^{\gamma-2}$ for some $\gamma>0$. For the application to the $2 \mathrm{~d}$ space white noise we could take $\gamma=1-$, but we will not use this specific information in order to probe the range of applicability of our approach and we will only assume that the exponent $\gamma$ is such that $3 \gamma-2>0$.

Assume for a moment that we are in the simpler situation $\gamma>1$ and $u_{0} \in \mathscr{C}^{\gamma}$ and let us try to solve equation (5.1) via Picard iterations $\left(u^{n}\right)_{n \geqslant 0}$ starting from $u^{0} \equiv u_{0}$. Since $F$ preserves the $C \mathscr{C}^{\gamma}$-regularity (which can be seen by identifying $C \mathscr{C}^{\gamma}$ with the classical space of bounded Höldercontinuous functions of space), the product $F\left(u^{0}(t)\right) \eta$ is well defined as an element of $\mathscr{C}^{\gamma-2}$ for all $t \geqslant 0$ since $2 \gamma-2>0$ and we are in condition to apply Corollary 1 below on the product of elements in Hölder-Besov spaces. Now by Lemma 11, the heat semigroup generated by the Laplacian gains two degrees of regularity so that the solution $u^{1}$ to $\mathscr{L} u^{1}=F\left(u^{0}\right) \eta$, $u^{1}(0)=u_{0}$, is in $C \mathscr{C}^{\gamma}$. From here we obtain a contraction on $C_{T} \mathscr{C}^{\gamma}$ for some small $T>0$ whose value does not depend on $u_{0}$, which gives us global in time existence and uniqueness of solutions. Note that in one dimension the space white noise has regularity $\mathscr{C}^{-1 / 2-}$ (see Exercise 11) so taking $\gamma=3 / 2-$ we have determined that the one-dimensional PAM can be solved globally in time with standard techniques. 
When the condition $2 \gamma-2>0$ is not satisfied we still have that if $\eta \in C_{T} \mathscr{C}^{\gamma-2}$ then $u \in \mathscr{L}^{\gamma}=C_{T} \mathscr{C}^{\gamma-2} \cap C_{T}^{\gamma / 2} L^{\infty}$ by the standard parabolic estimates of Lemma 11. However with the regularities at hand we cannot use Corollary 1 anymore to guarantee the continuity of the operator $(u, \eta) \mapsto F(u) \eta$. Moreover, as already seen in the simpler homogenization problems of Theorem 3 above, this is not a technical difficulty but a real issue of the regime $\gamma \leqslant 1$. We expect that controlling the model in this regime can be quite tricky since limits exists when $\eta \rightarrow 0$ but the limiting solution still feels residual order one effects from the vanishing driving signal $\eta$. This situation cannot be improved from the point of view of standard analytic considerations. What is needed is a finer control of the solution $u$ which allows to analyse in more detail the possible resonances between the fluctuations of $u$ and those of $\eta$.

Before going on we will revise the problem of multiplication of distributions in the scale of Hölder-Besov spaces, introducing the basic tool of our general analysis: Bony's paraproduct.

\subsection{The paraproduct and the resonant term}

Paraproducts are bilinear operations introduced by Bony [4] to linearize a class of nonlinear hyperbolic PDEs in order to analyse the regularity of their solutions. In terms of Littlewood-Paley blocks, a general product $f g$ of two distributions can be (at least formally) decomposed as

$$
f g=\sum_{j \geqslant-1} \sum_{i \geqslant-1} \Delta_{i} f \Delta_{j} g=f \prec g+f \succ g+f \circ g .
$$

Here $f \prec g$ is the part of the double sum with $i<j-1, f \succ g$ is the part with $i>j+1$, and $f \circ g$ is the "diagonal" part, where $|i-j| \leqslant 1$. More precisely, we define

$$
f \prec g=g \succ f=\sum_{j \geqslant-1} \sum_{i=-1}^{j-2} \Delta_{i} f \Delta_{j} g \quad \text { and } \quad f \circ g=\sum_{|i-j| \leqslant 1} \Delta_{i} f \Delta_{j} g .
$$

Of course, the decomposition depends on the dyadic partition of unity used to define the blocks $\Delta_{j}$, and also on the particular choice of the pairs $(i, j)$ in the diagonal part. The choice of taking all $(i, j)$ with $|i-j| \leqslant 1$ into the diagonal part corresponds to the fact that the partition of unity can be chosen such that supp $\mathscr{F}\left(\Delta_{i} f \Delta_{j} g\right) \subseteq 2^{j} \mathscr{A}$ if $i<j-1$, where $\mathscr{A}$ is a suitable annulus. If $|i-j| \leqslant 1$, the only apriori information on the spectral support of the various term in the double sum is supp $\mathscr{F}\left(\Delta_{i} f \Delta_{j} g\right) \subseteq 2^{j} \mathscr{B}$, 
that is they are supported in balls and in particular they can have nonzero contributions to very low wave vectors. We call $f \prec g$ and $f \succ g$ paraproducts, and $f \circ g$ the resonant term.

Bony's crucial observation is that $f \prec g$ (and thus $f \succ g$ ) is always a welldefined distribution. Heuristically, $f \prec g$ behaves at large frequencies like $g$ (and thus retains the same regularity), and $f$ provides only a frequency modulation of $g$. The only difficulty in constructing $f g$ for arbitrary distributions lies in handling the diagonal term $f \circ g$. The basic result about these bilinear operations is given by the following estimates.

Theorem 4. (Paraproduct estimates) For any $\beta \in \mathbb{R}$ and $f, g \in \mathscr{S}^{\prime}$ we have

$$
\|f \prec g\|_{\beta} \lesssim_{\beta}\|f\|_{L^{\infty}}\|g\|_{\beta},
$$

and for $\alpha<0$ furthermore

$$
\|f \prec g\|_{\alpha+\beta} \lesssim \alpha, \beta\|f\|_{\alpha}\|g\|_{\beta} .
$$

For $\alpha+\beta>0$ we have

$$
\|f \circ g\|_{\alpha+\beta} \lesssim \alpha, \beta\|f\|_{\alpha}\|g\|_{\beta} .
$$

Proof. There exists an annulus $\mathscr{A}$ such that $S_{j-1} f \Delta_{j} g$ has Fourier transform supported in $2^{j} \mathscr{A}$, and for $f \in L^{\infty}$ we have

$$
\left\|S_{j-1} f \Delta_{j} g\right\|_{L^{\infty}} \leqslant\left\|S_{j-1} f\right\|_{L^{\infty}}\left\|\Delta_{j} g\right\|_{L^{\infty}} \lesssim\|f\|_{L^{\infty}} 2^{-j \beta}\|g\|_{\beta} .
$$

By Lemma 10, we thus obtain (5.2). The proof of (5.3) and (5.4) works in the same way, where for estimating $f \circ g$ we need $\alpha+\beta>0$ because the terms of the series are supported in a ball and not in an annulus.

In combination with Exercise 10 above, we deduce the following simple corollary:

Corollary 1. Let $f \in \mathscr{C}^{\alpha}$ and $g \in \mathscr{C}^{\beta}$ with $\alpha+\beta>0$, then the product $(f, g) \mapsto f g$ is a bounded bilinear map from $\mathscr{C}^{\alpha} \times \mathscr{C}^{\beta}$ to $\mathscr{C}^{\alpha \wedge \beta}$. While $f \prec g, f \succ g$, and $f \circ g$ depend on the specific dyadic partition of unity, the product $f g$ does not.

The independence of the product from the dyadic partition of unity easily follows by taking smooth approximations.

The ill-posedness of $f \circ g$ for $\alpha+\beta \leqslant 0$ can be interpreted as a resonance effect since $f \circ g$ contains exactly those part of the double series where $f$ and $g$ are in the same frequency range. The paraproduct $f \prec g$ can be interpreted as frequency modulation of $g$, which should become more clear in the following example. 
Example 3. In Figure 5.1 we see a slowly oscillating positive function u, while Figure 5.2 depicts a fast sine curve $v$. The product uv, which here equals the paraproduct $u \prec v$ since $u$ has no rapidly oscillating components, is shown in Figure 5.3. We see that the local fluctuations of uv are due to $v$, and that uv is essentially oscillating with the same speed as $v$.
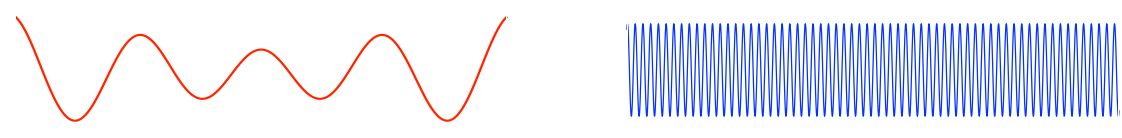

Figure 5.1: The function $u$

Figure 5.2: The function $v$

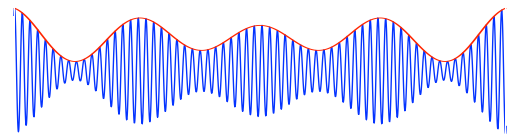

Figure 5.3: The function $u \prec v$

Example 4. If $f \in \mathscr{C}^{\gamma}(\mathbb{T})$ and $g \in \mathscr{C}^{\delta}(\mathbb{T})$ with $\gamma+\delta>1$, then we can define $\int f \mathrm{~d} g:=\int\left(f \partial_{t} g\right)$, which is well defined since $\partial_{t} g \in \mathscr{C}^{\delta-1}$ and $\gamma+\delta-1>0$, and since integration is a linear map. In this way we recover the Young integral [32].

Example 5. Let $B^{H}$ be a fractional Brownian bridge on $\mathbb{T}$ (or simply a fractional Brownian motion on $[0, \pi]$, reflected on $[\pi, 2 \pi]$ ) and assume that $H>1 / 2$. We have $\varphi\left(B^{H}\right) \in \mathscr{C}^{H-}$ for all Lipschitz continuous $\varphi$, and $\partial_{t} B^{H} \in \mathscr{C}^{(H-1)-}$, and in particular $\varphi\left(B^{H}\right) \partial_{t} B^{H}$ is well-defined. This can be used to solve SDEs driven by $B^{H}$ in a pathwise sense.

The condition $\alpha+\beta>0$ is essentially sharp, at least at this level of generality, see [32] for counterexamples. It excludes of course the Brownian case: if $B$ is a Brownian motion, then almost surely $B \in \mathscr{C}_{\text {loc }}^{\alpha}$ for all $\alpha<1 / 2$ (meaning that $\varphi B \in \mathscr{C}^{\alpha}$ whenever $\varphi$ is a smooth compactly supported function), so that $\partial_{t} B \in \mathscr{C}_{\mathrm{loc}}^{\alpha-1}$ and thus $B \circ \partial_{t} B$ fails to be well defined. See also [26], Proposition 1.29 for an instructive example which shows that this is not a shortcoming of our description of regularity, but that it is indeed impossible to define the product $B \partial_{t} B$ as a continuous bilinear operation on distribution spaces.

Other counterexamples are given by our discussion of the homogenization problem in Theorem 3 above. More simply, one can consider the following situation.

Example 6. Consider the sequence of functions $f_{n}: \mathbb{T} \rightarrow \mathbb{C}$ given by $f_{n}(x)=e^{i n^{2} x} / n$. Then it is easy to show that $\left\|f_{n}\right\|_{\gamma} \rightarrow 0$ for all $\gamma<1 / 2$. 
However let

$$
g_{n}(x)=\operatorname{Re} f_{n}(x) \operatorname{Im} \partial_{x} f_{n}(x)=\left(\cos \left(n^{2} x\right)\right)^{2}=\frac{\cos \left(2 n^{2} x\right)+1}{2}
$$

Then $g_{n} \rightarrow 1 / 2$ in $\mathscr{C}^{0-}$ which shows that the map $f \mapsto(\operatorname{Re} f)\left(\partial_{x} \operatorname{Im} f\right)$ cannot be continuous in $\mathscr{C}^{\gamma}$ if $\gamma<1 / 2$. Pictorially the situation is summarized in Figure 5.4, where we sketched the three dimensional curve given by $x \mapsto\left(\operatorname{Re} f_{n}(x), \operatorname{Im} f_{n}(x), \int_{0}^{x} g_{n}(y) \mathrm{d} y\right)$ for various values of $n$ and in the limit.

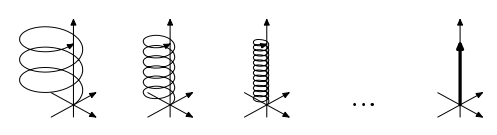

Figure 5.4: Resonances give macroscopic effects

\subsection{Commutator estimates and paralin- earization}

The product $F(u) \eta$ appearing in the right hand side of PAM can be decomposed via the paraproduct $\prec$ as a sum of three terms

$$
F(u) \eta=F(u) \prec \eta+F(u) \circ \eta+F(u) \succ \eta .
$$

The first and the last of these terms are continuous in any topology we will choose for $F(u)$ and $\eta$. The resonant term $F(u) \circ \eta$ however is problematic. It gathers the products of the oscillations of $F(u)$ and $\eta$ on comparable dyadic scales and these products can contribute to all larger scales in such a way that microscopic oscillations might build up to a macroscopic effect which does not disappear in the limit (as we have already seen in Theorem 3). If the function $F$ is smooth enough, then we expect the resonances between $F(u)$ and $\eta$ to correspond to the resonances between $u$ and $\eta$, and as we will see this is justified.

The expected regularity of the different terms is

$$
\underbrace{F(u) \prec \eta}_{\gamma-2}+\underbrace{F(u) \circ \eta}_{2 \gamma-2}+\underbrace{F(u) \succ \eta}_{2 \gamma-2},
$$

but unless $2 \gamma-2>0$ the resonant term $F(u) \circ \eta$ cannot be controlled using only the $C \mathscr{C}^{\gamma}$-norm of $u$ and the $C \mathscr{C}^{\gamma-2}-$ norm of $\eta$. If $F$ is at least 
$C^{2}$, we can use a paralinearization result (stated precisely in Lemma 16 below) to rewrite this term as

$$
F(u) \circ \eta=F^{\prime}(u)(u \circ \eta)+\Pi_{F}(u, \eta),
$$

with a remainder $\Pi_{F}(u, \eta) \in \mathscr{C}^{2 \gamma-2}$ provided $3 \gamma-2>0$. The difficulty is now localized in the linearized resonant product $u \circ \eta$. In order to control this term, we would like to exploit the fact that the function $u$ is not a generic element of $C \mathscr{C}^{\gamma}$ but that it has a specific structure, since $\mathscr{L} u$ has to match the paraproduct decomposition given in (5.5) where the least regular term is expected to be $F(u) \prec \eta \in C \mathscr{C}^{\gamma-2}$.

In order to do so, we postulate that the solution $u$ is given by the following paracontrolled ansatz:

$$
u=u^{X} \prec X+u^{\sharp},
$$

for functions $u^{X}, X, u^{\sharp}$ such that $u^{X}, X \in C \mathscr{C}^{\gamma}$ and the remainder $u^{\sharp} \in C \mathscr{C}^{2 \gamma}$. This decomposition allows for a finer analysis of the resonant term $u \circ \eta$ : indeed, we have

$$
u \circ \eta=\left(u^{X} \prec X\right) \circ \eta+u^{\sharp} \circ \eta=u^{X}(X \circ \eta)+C\left(u^{X}, X, \eta\right)+u^{\sharp} \circ \eta,
$$

where the commutator is defined by $C\left(u^{X}, X, \eta\right)=\left(u^{X} \prec X\right) \circ \eta-$ $u^{X}(X \circ \eta)$. Observe now that the term $u^{\sharp} \circ \eta$ does not pose any further problem, as it can be controlled in $C \mathscr{C}^{3 \gamma-2}$. The key point is now that the commutator is a bounded multilinear function of its arguments as long as the sum of their regularities is strictly positive, see Lemma 14 below. By assumption, we have $3 \gamma-2>0$, and therefore $C\left(u^{X}, X, \eta\right) \in C \mathscr{C}^{2 \gamma-2}$.

The only problematic term which remains to be handled is thus the bilinear functional of the noise given by $X \circ \eta$. Here we need to make the assumption that $X \circ \eta \in C \mathscr{C}^{2 \gamma-2}$ in order for the product $u^{X}(X \circ \eta)$ to be well defined. This assumption is not guaranteed by the analytical estimates at hand, and it has to be added as a further requirement to our construction.

Granting this last step, we have obtained that the right hand side of equation (5.1) is well defined and a continuous function of $\left(u, u^{X}, u^{\sharp}, X, \eta, X \circ \eta\right) \in C \mathscr{C}^{\gamma} \times C \mathscr{C}^{\gamma} \times C \mathscr{C}^{2 \gamma} \times C \mathscr{C}^{\gamma} \times C \mathscr{C}^{\gamma-2} \times C \mathscr{C}^{2 \gamma-2}$.

It remains to check that the paracontrolled ansatz is coherent with the equation satisfied by solutions to PAM. Let us first consider the linear example $F(u)=u$. Here we saw that the solution is of the form $u=e^{X} v$ with

$$
\mathscr{L} v=v\left|\partial_{x} X\right|^{2}+2\left\langle\partial_{x} v, \partial_{x} X\right\rangle_{\mathbb{R}^{2}}
$$

where $\left|\partial_{x} X\right|^{2} \in C \mathscr{C}^{2 \gamma-2}$ by Lemma 13 and $\partial_{x} X \in C \mathscr{C}^{\gamma-1}$ and therefore $v \in C \mathscr{C}^{2 \gamma}$ by the Bony and Schauder estimates. Note that here we have a 
clash of notation, because a priori the $X$ that we defined in Section 4 does not have to be equal to the paracontrolling distribution $X$. But of course, as the notation suggests, we will see momentarily that we can choose them to be the same. In the setting of Section 4, we have in particular

$$
u=e^{X} v=v \prec e^{X}+C \mathscr{C}^{2 \gamma}=v \prec\left(e^{X} \prec X\right)+C \mathscr{C}^{2 \gamma},
$$

where the notation $u=v \prec e^{X}+C \mathscr{C}^{2 \gamma}$ means that $u-v \prec e^{X} \in C \mathscr{C}^{2 \gamma}$, and where we used a paralinearization result in last step (see Lemma 15 below). Now the double paraproduct $f \prec(g \prec h)$ satisfies

$$
\|f \prec(g \prec h)-(f g) \prec h\|_{\alpha+\beta} \lesssim\|f\|_{\alpha}\|g\|_{\alpha}\|h\|_{\beta},
$$

see [4], and therefore $u=\left(v e^{X}\right) \prec X+C \mathscr{C}^{2 \gamma}=u \prec X+C \mathscr{C}^{2 \gamma}$ which shows that the paracontrolled ansatz is at least justified in the linear case and indeed we can choose the paracontrolling distribution to be $X$.

In the nonlinear case, the paracontrolled ansatz and the Leibniz rule for the paraproduct imply that (5.1) can be rewritten as

$$
\begin{aligned}
\mathscr{L} u & =\mathscr{L}\left(u^{X} \prec X+u^{\sharp}\right) \\
& =u^{X} \prec \mathscr{L} X+\left[\mathscr{L}, u^{X} \prec\right] X+\mathscr{L} u^{\sharp} \\
& =F(u) \prec \eta+F(u) \circ \eta+F(u) \succ \eta,
\end{aligned}
$$

where we recall that $\left[\mathscr{L}, u^{X} \prec\right] X=\mathscr{L}\left(u^{X} \prec X\right)-u^{X} \prec \mathscr{L} X$ denotes the commutator. If we choose $X$ such that $\mathscr{L} X=\eta$ and we set $u^{X}=F(u)$, then we can use (5.6) and (5.7) to obtain the following equation for the remainder $u^{\sharp}$ :

$$
\begin{aligned}
\mathscr{L} u^{\sharp}=F^{\prime}(u) F(u)(X \circ \eta)+F(u) \succ \eta-[\mathscr{L}, F(u) \prec] X \\
+F^{\prime}(u) C(F(u), X, \eta)+F^{\prime}(u)\left(u^{\sharp} \circ \eta\right)+\Pi_{F}(u, \eta) .
\end{aligned}
$$

Lemma 18 below ensures that $J[\mathscr{L}, F(u) \prec] X \in C \mathscr{C}^{2 \gamma}$ whenever $F(u) \in \mathscr{L}^{\gamma}$ (which easily follows from $u \in \mathscr{L}^{\gamma}$ by using the increment characterization of $\mathscr{C}^{\gamma}$ regularity), and combining the paraproduct estimates with the estimates for $C$ and $\Pi_{F}$ that we discussed above, we see that all the other terms on the right hand side are in $C \mathscr{C}^{2 \gamma-2}$. So the Schauder estimate Lemma 11 allows us to control $u^{\sharp}$ in $C \mathscr{C}^{2 \gamma}$. Together with $u=F(u) \prec X+u^{\sharp}$, equation (5.8) gives an equivalent description of the solution, because we only rewrote the original problem. This allows us to obtain a priori estimates on $u$ and $u^{\sharp}$ in terms of $\left(u_{0},\|\eta\|_{\gamma-2},\|X \circ \eta\|_{2 \gamma-2}\right)$, see Chapter 5 of [14] for details. It is now straightforward to show that if $F \in C_{b}^{3}$, then $u$ depends continuously on the data $\left(u_{0}, \eta, X \circ \eta\right)$, so that we have a robust strategy to pass to the limit in (4.5) and to make sense of the solution to (5.1) also for irregular $\eta \in C \mathscr{C}^{\gamma-2}$ as long as $\gamma>2 / 3$. 
In the remainder of this section we will prove the results (paralinearization and various key commutators) which we used in the discussion above, before going on to gather the consequences of our analysis in the next section. When the time dependence does not play any role we state the results for distributions depending only on the space variable as the extension to time varying functions will not add further difficulty.

Lemma 14. Assume that $\alpha, \beta, \gamma \in \mathbb{R}$ are such that $\alpha+\beta+\gamma>0$ and $\beta+\gamma \neq 0$. Then for $f, g, h \in C^{\infty}$ the trilinear operator

$$
C(f, g, h)=((f \prec g) \circ h)-f(g \circ h)
$$

satisfies

$$
\|C(f, g, h)\|_{\beta+\gamma} \lesssim\|f\|_{\alpha}\|g\|_{\beta}\|h\|_{\gamma},
$$

and can thus be uniquely extended to a bounded trilinear operator from $\mathscr{C}^{\alpha} \times \mathscr{C}^{\beta} \times \mathscr{C}^{\alpha}$ to $\mathscr{C}^{\beta+\gamma}$.

Proof. For $\beta+\gamma>0$ this follows from the paraproduct estimates, so let $\beta+\gamma<0$. By definition

$$
\begin{aligned}
C(f, g, h) & =\sum_{i, j, k, \ell} \Delta_{i}\left(\Delta_{j} f \Delta_{k} g\right) \Delta_{\ell} h\left(\mathbb{I}_{j<k-1} \mathbb{I}_{|i-\ell| \leqslant 1}-\mathbb{I}_{|k-\ell| \leqslant 1}\right) \\
& =\sum_{i, j, k, \ell} \Delta_{i}\left(\Delta_{j} f \Delta_{k} g\right) \Delta_{\ell} h\left(\mathbb{I}_{j<k-1} \mathbb{I}_{|i-\ell| \leqslant 1} \mathbb{I}_{|k-\ell| \leqslant N}-\mathbb{I}_{|k-\ell| \leqslant 1}\right),
\end{aligned}
$$

where we used that $\mathscr{F}\left(S_{k-1} f \Delta_{k} g\right)$ has support in an annulus $2^{k} \mathscr{A}$, so that $\Delta_{i}\left(S_{k-1} f \Delta_{k} g\right) \neq 0$ only if $|i-k| \leqslant N-1$ for some fixed $N \in \mathbb{N}$, which in combination with $|i-\ell| \leqslant 1$ yields $|k-\ell| \leqslant N$. Now the assumptions on our partition of unity guarantee that for fixed $k$, the term $\sum_{\ell} \mathbb{I}_{2 \leqslant|k-\ell| \leqslant N} \Delta_{k} g \Delta_{\ell} h$ is spectrally supported in an annulus $2^{k} \mathscr{A}$, so that $\sum_{k, \ell} \mathbb{I}_{2 \leqslant|k-\ell| \leqslant N} \Delta_{k} g \Delta_{\ell} h \in \mathscr{C}^{\beta+\gamma}$ and we may add and subtract $f \sum_{k, \ell} \mathbb{I}_{2 \leqslant|k-\ell| \leqslant N} \Delta_{k} g \Delta_{\ell} h$ to $C(f, g, h)$ while maintaining the bound (5.9). It remains to treat

$$
\begin{aligned}
& \sum_{i, j, k, \ell} \Delta_{i}\left(\Delta_{j} f \Delta_{k} g\right) \Delta_{\ell} h \mathbb{I}_{|k-\ell| \leqslant N}\left(\mathbb{I}_{j<k-1} \mathbb{I}_{|i-\ell| \leqslant 1}-1\right) \\
= & -\sum_{i, j, k, \ell} \Delta_{i}\left(\Delta_{j} f \Delta_{k} g\right) \Delta_{\ell} h \mathbb{I}_{|k-\ell| \leqslant N}\left(\mathbb{I}_{j \geqslant k-1}+\mathbb{I}_{j<k-1} \mathbb{I}_{|i-\ell|>1}\right) .
\end{aligned}
$$

We estimate both terms on the right hand side separately. For $m \geqslant-1$ we have (recall that for indices of Littlewood-Paley blocks, $i \lesssim j$ is to be 
read as $2^{i} \lesssim 2^{j}$, that is $i \leq j+c$ for some fixed $c$ ):

$$
\begin{aligned}
& \left\|\Delta_{m}\left(\sum_{i, j, k, \ell} \Delta_{i}\left(\Delta_{j} f \Delta_{k} g\right) \Delta_{\ell} h \mathbb{I}_{|k-\ell| \leqslant N} \mathbb{I}_{j \geqslant k-1}\right)\right\|_{L^{\infty}} \\
\leqslant & \sum_{j, k, \ell} \mathbb{I}_{|k-\ell| \leqslant N} \mathbb{I}_{j \geqslant k-1}\left\|\Delta_{m}\left(\Delta_{j} f \Delta_{k} g \Delta_{\ell} h\right)\right\|_{L^{\infty}} \\
\lesssim & \sum_{j \gtrsim m} \sum_{k \lesssim j} 2^{-j \alpha}\|f\|_{\alpha} 2^{-k \beta}\|g\|_{\beta} 2^{-k \gamma}\|h\|_{\gamma} \\
\lesssim & \sum_{j \gtrsim m} 2^{-j(\alpha+\beta+\gamma)}\|f\|_{\alpha}\|g\|_{\beta}\|h\|_{\gamma} \lesssim 2^{-m(\alpha+\beta+\gamma)}\|f\|_{\alpha}\|g\|_{\beta}\|h\|_{\gamma},
\end{aligned}
$$

using $\beta+\gamma<0$ to get $\sum_{k \lesssim j} 2^{k(\beta+\gamma)} \lesssim 2^{j(\alpha+\beta)}$. It remains to estimate the second term in (5.10). For $|i-\ell|>1$ and $i \sim k \sim \ell$, any term of the form $\Delta_{i}(\cdot) \Delta_{\ell}(\cdot)$ is spectrally supported in an annulus $2^{\ell} \mathscr{A}$, and therefore

$$
\begin{aligned}
& \left\|\Delta_{m}\left(\sum_{i, j, k, \ell} \Delta_{i}\left(\Delta_{j} f \Delta_{k} g\right) \Delta_{\ell} h \mathbb{I}_{|k-\ell| \leqslant N} \mathbb{I}_{j<k-1} \mathbb{I}_{|i-\ell|>1}\right)\right\|_{L^{\infty}} \\
\lesssim & \sum_{i, j, k, \ell} \mathbb{I}_{j<k-1} \mathbb{I}_{i \sim k \sim \ell \sim m}\left\|\Delta_{i}\left(\Delta_{j} f \Delta_{k} g\right) \Delta_{\ell} h\right\|_{L^{\infty}} \\
\lesssim & \sum_{j \lesssim m} 2^{-j \alpha}\|f\|_{\alpha} 2^{-m \beta}\|g\|_{\beta} 2^{-m \gamma}\|h\|_{\gamma} \lesssim 2^{-m(\beta+\gamma)}\|f\|_{\alpha}\|g\|_{\beta}\|h\|_{\gamma} .
\end{aligned}
$$

Remark 6. For $\beta+\gamma=0$ we can apply the commutator estimate with $\gamma^{\prime}<\gamma$, as long as $\alpha+\beta+\gamma^{\prime}>0$.

Our next result is a simple paralinearization lemma for non-linear operators.

Lemma 15 (see also [1], Theorem 2.92). Let $\alpha \in(0,1), \beta \in(0, \alpha]$, and let $F \in C_{b}^{1+\beta / \alpha}$. There exists a locally bounded map $R_{F}: \mathscr{C}^{\alpha} \rightarrow \mathscr{C}^{\alpha+\beta}$ such that

$$
F(f)=F^{\prime}(f) \prec f+R_{F}(f)
$$

for all $f \in \mathscr{C}^{\alpha}$. More precisely, we have

$$
\left\|R_{F}(f)\right\|_{\alpha+\beta} \lesssim\|F\|_{C_{b}^{1+\beta / \alpha}}\left(1+\|f\|_{\alpha}^{1+\beta / \alpha}\right) .
$$

If $F \in C_{b}^{2+\beta / \alpha}$, then $R_{F}$ is locally Lipschitz continuous:

$$
\left\|R_{F}(f)-R_{F}(g)\right\|_{\alpha+\beta} \lesssim\|F\|_{C_{b}^{2+\beta / \alpha}}\left(1+\|f\|_{\alpha}+\|g\|_{\alpha}\right)^{1+\beta / \alpha}\|f-g\|_{\alpha} .
$$


Remark 7. Since every element of $\mathscr{C}^{\alpha}$ is bounded, the result immediately extends to unbounded $F \in C^{1+\beta / \alpha}$ : Simply replace $F$ by an element of $C_{b}^{1+\beta / \alpha}$ which agrees with $F$ on the image of $f$.

Proof. [Proof of Lemma 15] The difference $F(f)-F^{\prime}(f) \prec f$ is given by

$$
R_{F}(f)=F(f)-F^{\prime}(f) \prec f=\sum_{i \geqslant-1}\left[\Delta_{i} F(f)-S_{i-1} F^{\prime}(f) \Delta_{i} f\right]=\sum_{i \geqslant-1} u_{i},
$$

and every $u_{i}$ is spectrally supported in a ball $2^{i} \mathscr{B}$. For $i<1$, we simply estimate $\left\|u_{i}\right\|_{L^{\infty}} \lesssim\|F\|_{C_{b}^{1}}\left(1+\|f\|_{\alpha}\right)$. For $i \geqslant 1$ we use the fact that $f$ is a bounded function to write the Littlewood-Paley projections as convolutions and obtain

$$
\begin{aligned}
& u_{i}(x) \\
= & \int K_{i}(x-y) K_{<i-1}(x-z)\left[F(f(y))-F^{\prime}(f(z)) f(y)\right] \mathrm{d} y \mathrm{~d} z \\
= & \int K_{i}(x-y) K_{<i-1}(x-z)\left[F(f(y))-F(f(z))-F^{\prime}(f(z))(f(y)-f(z))\right] \mathrm{d} y \mathrm{~d} z,
\end{aligned}
$$

where $K_{i}=\mathscr{F}^{-1} \rho_{i}, K_{<i-1}=\sum_{j<i-1} K_{j}$, and where we used that $\int K_{i}(y) \mathrm{d} y=\rho_{i}(0)=0$ for $i \geqslant 0$ and $\int K_{<i-1}(z) \mathrm{d} z=1$ for $i \geqslant 1$. Now we can apply a first order Taylor expansion to $F$ and use the $\beta / \alpha$-Hölder continuity of $F^{\prime}$ in combination with the $\alpha$-Hölder continuity of $f$, to deduce

$$
\begin{aligned}
&\left|u_{i}(x)\right| \\
& \lesssim\|F\|_{C_{b}^{1+\beta / \alpha}}\|f\|_{\alpha}^{1+\beta / \alpha} \int\left|K_{i}(x-y) K_{<0}(x-z)\right| \times|z-y|^{\alpha+\beta} \mathrm{d} y \mathrm{~d} z \\
&=\|F\|_{C_{b}^{1+\beta / \alpha}}\|f\|_{\alpha}^{1+\beta / \alpha} 2^{-(i-1)(\alpha+\beta)} \\
& \times \int\left|2^{(i-1) d} K_{1}\left(2^{i-1}(x-y)\right) 2^{(i-1) d} K_{<0}\left(2^{i-1}(x-z)\right)\right| \times\left|2^{i-1}(z-y)\right|^{\alpha+\beta} \mathrm{d} y \mathrm{~d} z \\
& \lesssim\|F\|_{C_{b}^{1+\beta / \alpha}}\|f\|_{\alpha}^{1+\beta / \alpha} 2^{-i(\alpha+\beta)} .
\end{aligned}
$$

Therefore, the estimate for $R_{F}(f)$ follows from Lemma 10. The estimate for $R_{F}(f)-R_{F}(g)$ is shown in the same way.

Let $g$ be a distribution belonging to $\mathscr{C}^{\beta}$ for some $\beta<0$. Then the map $f \mapsto f \circ g$ behaves, modulo smoother correction terms, like a derivative operator:

Lemma 16. Let $\alpha \in(0,1), \beta \in(0, \alpha], \gamma \in \mathbb{R}$ be such that $\alpha+\beta+\gamma>0$ and $\alpha+\gamma \neq 0$. Let $F \in C_{b}^{1+\beta / \alpha}$. Then there exists a locally bounded map $\Pi_{F}: \mathscr{C}^{\alpha} \times \mathscr{C}^{\gamma} \rightarrow \mathscr{C}^{\alpha+\gamma}$ such that

$$
F(f) \circ g=F^{\prime}(f)(f \circ g)+\Pi_{F}(f, g)
$$


for all $f \in \mathscr{C}^{\alpha}$ and all smooth $g$. More precisely, we have

$$
\left\|\Pi_{F}(f, g)\right\|_{\alpha+\gamma} \lesssim\|F\|_{C_{b}^{1+\beta / \alpha}}\left(1+\|f\|_{\alpha}^{1+\beta / \alpha}\right)\|g\|_{\gamma} .
$$

If $F \in C_{b}^{2+\beta / \alpha}$, then $\Pi_{F}$ is locally Lipschitz continuous:

$$
\begin{aligned}
& \left\|\Pi_{F}(f, g)-\Pi_{F}(u, v)\right\|_{\alpha+\gamma} \\
\lesssim & \|F\|_{C_{b}^{2+\beta / \alpha}}\left(1+\|f\|_{\alpha}+\|u\|_{\alpha}\right)^{1+\beta / \alpha}\left(1+\|v\|_{\gamma}\right)\left(\|f-u\|_{\alpha}+\|g-v\|_{\gamma}\right) .
\end{aligned}
$$

Proof. Use the paralinearization and commutator lemmas above to deduce that

$$
\begin{aligned}
\Pi_{F}(f, g) & =F(f) \circ g-F^{\prime}(f)(f \circ g) \\
& =R_{F}(f) \circ g+\left(F^{\prime}(f) \prec f\right) \circ g-F^{\prime}(f)(f \circ g) \\
& =R_{F}(f) \circ g+C\left(F^{\prime}(f), f, g\right),
\end{aligned}
$$

so that the claimed bounds easily follow from Lemma 14 and Lemma 15.

Besides this sort of chain rule, we also have a Leibniz rule for $f \mapsto f \circ g$ :

Lemma 17. Let $\alpha \in(0,1)$ and $\gamma<0$ be such that $2 \alpha+\gamma>0$ and $\alpha+\gamma \neq 0$. Then there exists a bounded trilinear operator $\Pi_{\times}: \mathscr{C}^{\alpha} \times \mathscr{C}^{\alpha} \times \mathscr{C}^{\gamma} \rightarrow \mathscr{C}^{\alpha+\gamma}$, such that

$$
(f u) \circ g=f(u \circ g)+u(f \circ g)+\Pi_{\times}(f, u, g)
$$

for all $f, u \in \mathscr{C}^{\alpha}(\mathbb{R})$ and all smooth $g$.

Proof. It suffices to note that $f u=f \prec u+f \succ u+f \circ u$, which leads to $\Pi_{\times}(f, u, g)=(f u) \circ g-f(u \circ g)-u(f \circ g)=C(f, u, g)+C(u, f, g)+(f \circ u) \circ g$.

Lemma 18. Let $\beta<1, \alpha \in \mathbb{R}$, and let $f \in \mathscr{L}^{\beta}$ and $G \in C \mathscr{C}^{\alpha}$ with $\mathscr{L} G \in C \mathscr{C}^{\alpha-2}$. There exists $H=H(f, G)$ such that $\mathscr{L} H=[\mathscr{L}, f \prec] G$ and $H(0)=0$. Moreover $H \in C \mathscr{C}^{\alpha+\beta} \cap C^{(\alpha \wedge \beta) / 2} L^{\infty}$ and for all $T>0$

$$
\|H\|_{C_{T}^{(\alpha \wedge \beta) / 2} L^{\infty}}+\|H\|_{C_{T} \mathscr{C}^{\alpha+\beta}} \lesssim\|f\|_{\mathscr{L}_{T}^{\beta}}\left(\|G\|_{C_{T} \mathscr{C}^{\alpha}}+\|\mathscr{L} G\|_{C_{T} \mathscr{C}^{\alpha-2}}\right) .
$$

Proof. Let $T>0$ and let $f_{\varepsilon}$ be a time mollification of $f$ such that $\left\|\partial_{t} f_{\varepsilon}\right\|_{C_{T} L^{\infty}} \lesssim \varepsilon^{\beta / 2-1}\|f\|_{\mathscr{L}_{T}^{\beta}}$ and $\left\|f_{\varepsilon}-f\right\|_{C_{T} L^{\infty}} \lesssim \varepsilon^{\beta / 2}\|f\|_{\mathscr{L}^{\beta}}$ for all $\varepsilon>0$. For example we can take $f_{\varepsilon}=\rho_{\varepsilon} * f$ with $\rho_{\varepsilon}(t)=\rho(t / \varepsilon) / \varepsilon$ and $\rho: \mathbb{R} \rightarrow \mathbb{R}$ compactly supported, smooth, and of unit integral. For $i \geqslant-1$ we have

$$
\begin{aligned}
\mathscr{L} \Delta_{i} H & =\Delta_{i} \mathscr{L} H \\
& =\Delta_{i}\left[\mathscr{L}\left(\left(f-f_{\varepsilon}\right) \prec G\right)-\left(f-f_{\varepsilon}\right) \prec \mathscr{L} G\right]+\Delta_{i}\left[\mathscr{L}\left(f_{\varepsilon} \prec G\right)-f_{\varepsilon} \prec \mathscr{L} G\right],
\end{aligned}
$$


so that

$$
\begin{aligned}
\mathscr{L} \Delta_{i}\left(H-\left(f-f_{\varepsilon}\right) \prec G\right) & =-\Delta_{i}\left[\left(f-f_{\varepsilon}\right) \prec \mathscr{L} G\right]+\Delta_{i}\left[\mathscr{L}\left(f_{\varepsilon} \prec G\right)-f_{\varepsilon} \prec \mathscr{L} G\right] \\
& =\Delta_{i}\left[\left(f_{\varepsilon}-f\right) \prec \mathscr{L} G\right]+\Delta_{i}\left[\mathscr{L} f_{\varepsilon} \prec G-2 \partial_{x} f_{\varepsilon} \prec \partial_{x} G\right],
\end{aligned}
$$

with initial condition $\Delta_{i}\left(H-\left(f-f_{\varepsilon}\right) \prec G\right)(0)=-\left(\Delta_{i}\left(f-f_{\varepsilon}\right) \prec G\right)(0)$.

The Schauder estimates for $\mathscr{L}$ (Lemma 11) give

$$
\begin{aligned}
&\left\|\Delta_{i}\left(H+\left(f-f_{\varepsilon}\right) \prec G\right)\right\|_{\mathscr{L}_{T}^{\alpha+\beta}} \\
& \lesssim\left\|\Delta_{i}\left[\left(f-f_{\varepsilon}\right) \prec \mathscr{L} G\right]+\Delta_{i}\left[\left(\mathscr{L} f_{\varepsilon}\right) \prec G-2 \partial_{x} f_{\varepsilon} \prec \partial_{x} G\right]\right\|_{C_{T} \mathscr{C}^{\alpha+\beta-2}} \\
& \quad+\left\|\left(\Delta_{i}\left(f-f_{\varepsilon}\right) \prec G\right)(0)\right\|_{\alpha+\beta} .
\end{aligned}
$$

Choosing $\varepsilon=2^{-2 i}$, we have

$$
\begin{aligned}
\left\|\Delta_{i}\left(\left(f-f_{\varepsilon}\right) \prec G\right)\right\|_{C_{T} \mathscr{C}^{\alpha+\beta}} & \lesssim 2^{\beta i}\left\|\Delta_{i}\left(\left(f-f_{\varepsilon}\right) \prec G\right)\right\|_{C_{T} \mathscr{C}^{\alpha}} \\
& \lesssim 2^{\beta i}\left\|f-f_{\varepsilon}\right\|_{C_{T} L^{\infty}}\|G\|_{C_{T} \mathscr{C}^{\alpha}} \\
& \lesssim\|f\|_{\mathscr{L}_{T}^{\beta}}\|G\|_{C_{T} \mathscr{C}^{\alpha}}
\end{aligned}
$$

and exactly the same argument also gives

$$
\left\|\Delta_{i}\left[\left(f-f_{\varepsilon}\right) \prec \mathscr{L} G\right]\right\|_{C_{T} \mathscr{C}^{\alpha+\beta-2}} \lesssim\|f\|_{\mathscr{L}_{T}^{\beta}}\|\mathscr{L} G\|_{C_{T} \mathscr{C}^{\alpha-2}} .
$$

Since $\beta<1$, we further get

$$
\begin{aligned}
& \left\|\Delta_{i}\left[\mathscr{L} f_{\varepsilon} \prec G+\partial_{x} f_{\varepsilon} \prec \partial_{x} G\right]\right\|_{C_{T} \mathscr{C}^{\alpha+\beta-2}} \\
\lesssim & 2^{i(\beta-2)}\left\|\partial_{t} f_{\varepsilon}\right\|_{C_{T} L^{\infty}}\|G\|_{C_{T} \mathscr{C}^{\alpha}}+\left\|f_{\varepsilon}\right\|_{C_{T} \mathscr{C}^{\beta}}\|G\|_{C_{T} \mathscr{C}^{\alpha}} \\
\lesssim & \|f\|_{\mathscr{L}_{T}^{\beta}}\|G\|_{C_{T} \mathscr{C}^{\alpha}}+\|f\|_{C_{T} \mathscr{C}^{\beta}}\|G\|_{C_{T} \mathscr{C}^{\alpha}} .
\end{aligned}
$$

Combining everything, we end up with

$$
\left\|\Delta_{i} H\right\|_{C_{T} \mathscr{C}^{\alpha+\beta}} \lesssim\|f\|_{\mathscr{L}_{T}^{\beta}}\left(\|G\|_{C_{T} \mathscr{C}^{\alpha}}+\|\mathscr{L} G\|_{C_{T} \mathscr{C}^{\alpha-2}}\right),
$$

which gives the estimate for the space regularity of $H$ since $\left\|\Delta_{i} H\right\|_{C_{T} L^{\infty}} \lesssim$ $2^{-(\alpha+\beta) i}\left\|\Delta_{i} H\right\|_{C_{T} \mathscr{C}^{\alpha+\beta}}$. The time regularity of $H$ can be controlled similarly by noting that $\left(f-f_{\varepsilon}\right) \prec G \in C_{T}^{(\alpha \wedge \beta) / 2} L^{\infty}$, uniformly in $\varepsilon$.

\subsection{Paracontrolled distributions}

Here we build a calculus of distributions satisfying a paracontrolled ansatz. We start by defining a suitable space of such objects.

Definition 5. Let $\alpha>0$ and $\beta \in(0, \alpha]$ be such that $\alpha+\beta \in(0,2)$, and let $u \in \mathscr{L}^{\alpha}$. A pair of distributions $\left(f, f^{u}\right) \in \mathscr{L}^{\alpha} \times \mathscr{L}^{\beta}$ is called 
paracontrolled by $u$ if $f^{\sharp}=f-f^{u} \prec u \in C \mathscr{C}^{\alpha+\beta} \cap \mathscr{L}^{\beta}$. In that case we write $f \in \mathscr{D}^{\beta}=\mathscr{D}^{\beta}(u)$, and for all $T>0$ we define the norm

$$
\|f\|_{\mathscr{D}_{T}^{\beta}}=\|f\|_{C_{T}^{\alpha / 2}}+\left\|f^{u}\right\|_{\mathscr{L}_{T}^{\beta}}+\left\|f^{\sharp}\right\|_{C_{T} \mathscr{C}^{\alpha+\beta}}+\left\|f^{\sharp}\right\|_{C_{T}^{\beta / 2} L^{\infty}} .
$$

If $\tilde{u} \in \mathscr{L}^{\alpha}$ and $\left(\tilde{f}, \tilde{f}^{\tilde{u}}\right) \in \mathscr{D}^{\beta}(\tilde{u})$, then we also write

$$
d_{\mathscr{D}_{T}^{\beta}}(f, \tilde{f})=\left\|f^{u}-\tilde{f}^{\tilde{u}}\right\|_{\mathscr{L}_{T}^{\beta}}+\left\|f^{\sharp}-\tilde{f}^{\sharp}\right\|_{C_{T} \mathscr{C}^{\alpha+\beta}}+\left\|f^{\sharp}-\tilde{f}^{\sharp}\right\|_{C_{T}^{\beta / 2} L^{\infty}} .
$$

Note that in general $f$ and $\tilde{f}$ do not live on the same space, so $d_{\mathscr{D}_{T}^{\beta}}$ is not a distance.

Of course we should really write $\left(f, f^{u}\right) \in \mathscr{D}^{\beta}$ since given $f$ and $u$, the derivative $f^{u}$ is usually not uniquely determined. But in the applications there will always be an obvious candidate for the derivative, and no confusion will arise.

Remark 8. The space $\mathscr{D}^{\beta}$ does not depend on the specific dyadic partition of unity. Indeed, Bony [4] has shown that if $\widetilde{\prec}$ is the paraproduct constructed from another partition of unity, then $\| f^{u} \prec u-$ $f^{u} \approx u\left\|_{C_{T} \mathscr{C}^{\alpha+\beta}} \lesssim\right\| f^{u}\left\|_{C_{T} \mathscr{C}^{\beta}}\right\| u \|_{C_{T} \mathscr{C}^{\alpha}}$.

Nonlinear operations As an immediate consequence of Lemma 14 we can multiply any distribution that is paracontrolled by $u$ with a given $v$, provided that we know how to multiply $u$ with $v$ (of course always under suitable regularity assumptions):

Theorem 5 (also see Theorem 3.7 of [14]). Let $\alpha, \beta \in \mathbb{R}, \gamma<0$, with $\alpha+\beta+\gamma>0$ and $\alpha+\gamma \neq 0$. Let $u \in C \mathscr{C}^{\alpha}, v \in C \mathscr{C}^{\gamma}$, and let $\zeta \in C \mathscr{C}^{\alpha+\gamma}$. Then

$$
\mathscr{D}^{\beta}(u) \ni f \mapsto f \cdot v:=f \prec v+f \succ v+f^{\sharp} \circ v+C\left(f^{u}, u, v\right)+f^{u} \zeta \in C \mathscr{C}^{\gamma}
$$

defines a bounded linear operator and for all $T>0$ we have the bound

$$
\begin{aligned}
\left\|(f v)^{\sharp}\right\|_{C_{T} \mathscr{C}^{\alpha+\gamma}} & :=\|f \cdot v-f \prec v\|_{C_{T} \mathscr{C}^{\alpha+\gamma}} \\
& \lesssim\|f\|_{\mathscr{D}_{T}^{\beta}}\left(\|v\|_{C_{T} \mathscr{C}^{\gamma}}+\|u\|_{C_{T} \mathscr{C}^{\alpha}}\|v\|_{C_{T} \mathscr{C} \gamma}+\|\zeta\|_{C_{T} \mathscr{C}^{\alpha+\gamma}}\right) .
\end{aligned}
$$

If there exist sequences of smooth functions $\left(u_{n}\right)$ and $\left(v_{n}\right)$ converging to $u$ and $v$ in $C \mathscr{C}^{\alpha}$ and $C \mathscr{C}^{\gamma}$ respectively for which $\left(u_{n} \circ v_{n}\right)$ converges to $\zeta$ in $C \mathscr{C}^{\alpha+\gamma}$, then $f \cdot v$ does not depend on the dyadic partition of unity used to construct it.

Furthermore, there exists a quadratic polynomial $P$ so that if $\tilde{u}, \tilde{v}, \tilde{\zeta}$ satisfy the same assumptions as $u, v, \zeta$ respectively, if $\tilde{f} \in \mathscr{D}^{\beta}(\tilde{u})$, and 
if

$$
\begin{array}{r}
M=\max \left\{\|u\|_{C_{T} \mathscr{C}^{\alpha}},\|v\|_{C_{T} \mathscr{C}^{\gamma}},\|\zeta\|_{C_{T} \mathscr{C}^{\alpha+\gamma}},\|\tilde{u}\|_{C_{T} \mathscr{C}^{\alpha}},\|\tilde{v}\|_{C_{T} \mathscr{C}^{\gamma}}\right. \\
\left.\|\tilde{\zeta}\|_{C_{T} \mathscr{C}^{\alpha+\gamma}},\|f\|_{\mathscr{D}_{T}^{\beta}(u)},\|\tilde{f}\|_{\mathscr{D}_{T}^{\beta}(\tilde{u})}\right\},
\end{array}
$$

then

$$
\begin{aligned}
& \left\|(f v)^{\sharp}-(\tilde{f} \tilde{v})^{\sharp}\right\|_{C_{T} \mathscr{C}^{\alpha+\gamma}} \\
\leqslant & P(M)\left(d_{\mathscr{D}^{\beta}}(f, \tilde{f})+\|u-\tilde{u}\|_{C_{T} \mathscr{C}^{\alpha}}+\|v-\tilde{v}\|_{C_{T} \mathscr{C}^{\gamma}}+\|\zeta-\tilde{\zeta}\|_{C_{T} \mathscr{C}^{\alpha+\gamma}}\right) .
\end{aligned}
$$

Proof. Given Lemma 14 (and the paraproduct estimates Theorem 4), the proof is straightforward and we leave most of it as an exercise. Let us only comment on the independence of the partition of unity: let $\left(u^{n}, v^{n}\right)$ be as announced and define $f_{n}:=f^{u} \prec u_{n}+f^{\sharp}$. Then

$$
\begin{aligned}
\lim _{n \rightarrow \infty} f_{n} v_{n} & =\lim _{n \rightarrow \infty}\left(f_{n} \prec v_{n}+f_{n} \succ v_{n}+f^{\sharp} \circ v_{n}+C\left(f^{u}, u_{n}, v_{n}\right)+f^{u}\left(u_{n} \circ v_{n}\right)\right) \\
& =f \prec v+f \succ v+f^{\sharp} \circ v+C\left(f^{u}, u, v\right)+f^{u} \zeta=f \cdot v .
\end{aligned}
$$

Since the pointwise product $f_{n} v_{n}$ does not depend on the partition of unity, also the limit must be independent.

The bound on the difference is obtained by using the boundedness and multilinearity of all operators involved.

From now on we will assume that there exist smooth functions $\left(u_{n}\right)$ and $\left(v_{n}\right)$ converging to $u$ and $v$ respectively for which $\left(u_{n} \circ v_{n}\right)$ converges to $\zeta$, so that the product does not depend on the partition of unity, and we will usually write $f v$ rather than $f \cdot v$. Later we will see that the resonant term $\left(u_{n} \circ v_{n}\right)$ must often be renormalized by subtracting a large constant, but this will not affect the independence of the product from the partition of unity.

To solve equations involving general nonlinear functions, we need to examine the stability of paracontrolled distributions under smooth functions.

Theorem 6. Let $\alpha \in(0,1)$ and $\beta \in(0, \alpha]$. Let $u \in \mathscr{L}^{\alpha}, f \in \mathscr{D}^{\alpha}(u)$, and $F \in C_{b}^{1+\beta / \alpha}$. Then $F(f) \in \mathscr{D}^{\beta}$ with derivative $(F(f))^{u}=F^{\prime}(f) f^{u}$, and for all $T>0$

$$
\|F(f)\|_{\mathscr{D}_{T}^{\beta}} \lesssim\|F\|_{C_{b}^{1+\beta / \alpha}}\left(1+\|f\|_{\mathscr{D}_{T}^{\alpha}}^{2}\right)\left(1+\|u\|_{\mathscr{L}_{T}^{\alpha}}^{2}\right) .
$$

Moreover, there exists a polynomial $P$ which satisfies, for all $F \in C_{b}^{2+\beta / \alpha}$, $\tilde{u} \in \mathscr{L}^{\alpha}, \tilde{f} \in \mathscr{D}^{\alpha}(\tilde{u})$, and

$$
M:=\max \left\{\|u\|_{\mathscr{L}_{T}^{\alpha}},\|\tilde{u}\|_{\mathscr{L}_{T}^{\alpha}},\|f\|_{\mathscr{D}_{T}^{\alpha}(u)},\|\tilde{f}\|_{\mathscr{D}_{T}^{\alpha}(\tilde{u})}\right\},
$$


the bound

$$
d_{\mathscr{D}_{T}^{\beta}}(F(f), F(\tilde{f})) \leqslant P(M)\|F\|_{C_{T}^{2+\beta / \alpha}}\left(d_{\mathscr{D}_{T}^{\alpha}}(f, \tilde{f})+\|u-\tilde{u}\|_{\mathscr{L}_{T}^{\alpha}}\right) .
$$

The proof is not very complicated but rather lengthy, and we do not present it here. The reader can find it in [14].

Schauder estimate for paracontrolled distributions The Schauder estimate Lemma 11 is not quite sufficient: we also need to understand how the heat kernel acts on the paracontrolled structure.

Theorem 7. Let $\alpha \in(0,1)$ and $\beta \in(0, \alpha]$. Let $u \in C \mathscr{C}^{\alpha-2}$ and $\mathscr{L} U=u$ with $U(0)=0$. Let $f^{u} \in \mathscr{L}^{\beta}, f^{\sharp} \in C \mathscr{C}^{\alpha+\beta-2}$, and $g_{0} \in \mathscr{C}^{\alpha+\beta}$. Then $\left(g, f^{u}\right) \in \mathscr{D}^{\beta}(U)$, where $g$ solves

$$
\mathscr{L} g=f^{u} \prec u+f^{\sharp}, \quad g(0)=g_{0},
$$

and we have the bound

$\|g\|_{\mathscr{D}_{T}^{\beta}(U)} \lesssim\left\|g_{0}\right\|_{\alpha+\beta}+(1+T)\left(\left\|f^{u}\right\|_{\mathscr{L}_{T}^{\beta}}\left(1+\|u\|_{C_{T} \mathscr{C}^{\alpha-2}}\right)+\left\|f^{\sharp}\right\|_{C_{T} \mathscr{C}^{\alpha+\beta-2}}\right)$

for all $T>0$. If furthermore $\tilde{u}, \tilde{U}, \tilde{f} \tilde{u}, \tilde{f}^{\sharp}, \tilde{g}_{0}, \tilde{g}$ satisfy the same assumptions as $u, U, f^{u}, f^{\sharp}, g_{0}, g$ respectively, and if $M=$ $\max \left\{\left\|f^{u}\right\|_{\mathscr{L}_{T}^{\beta}},\|\tilde{u}\|_{C_{T} \mathscr{C}^{\alpha-2}}, 1\right\}$, then

$$
\begin{aligned}
d_{\mathscr{D}_{T}^{\beta}}(g, \tilde{g}) \lesssim & \left\|g_{0}-\tilde{g}_{0}\right\|_{\alpha+\beta} \\
& +(1+T) M\left(\left\|f^{u}-\tilde{f}^{\tilde{u}}\right\|_{\mathscr{L}_{T}^{\beta}}+\|u-\tilde{u}\|_{C_{T} \mathscr{C}^{\alpha-2}}+\left\|f^{\sharp}-\tilde{f}^{\sharp}\right\|_{C_{T} \mathscr{C}^{\alpha+\beta-2}}\right) .
\end{aligned}
$$

Proof. Let us derive an equation for the remainder $g^{\sharp}$. We have

$$
\begin{aligned}
\mathscr{L} g^{\sharp} & =\mathscr{L} g-\mathscr{L}\left(f^{\prime} \prec U\right) \\
& =\left[f^{u} \prec u+f^{\sharp}\right]-f^{u} \prec \mathscr{L} U-\left[\mathscr{L}\left(f^{u} \prec U\right)-f^{u} \prec \mathscr{L} U\right] \\
& =f^{\sharp}-\left[\mathscr{L}, f^{u} \prec\right] U .
\end{aligned}
$$

Since $\alpha \wedge \beta=\beta$ we can now apply Lemma 18 to see that there exists $H \in C \mathscr{C}^{\alpha+\beta} \cap C^{\beta / 2} L^{\infty}$ such that $\mathscr{L} H=\left[\mathscr{L}, f^{u} \prec\right] U$, so we can apply the standard Schauder estimates of Lemma 11 to $\mathscr{L}\left(g^{\sharp}+H\right)=f^{\sharp}$ to get

$$
\begin{gathered}
\left\|g^{\sharp}\right\|_{C_{T} \mathscr{C}^{\alpha+\beta}}+\left\|g^{\sharp}\right\|_{C_{T}^{(\alpha \wedge \beta) / 2} L^{\infty}} \lesssim\left\|f^{u}\right\|_{\mathscr{L}_{T}^{\beta}}\left(\|U\|_{C_{T} \mathscr{C}^{\alpha}}+\|\mathscr{L} U\|_{C_{T} \mathscr{C}^{\alpha-2}}\right) \\
+\left\|f^{\sharp}\right\|_{C_{T} \mathscr{C}^{\alpha+\beta-2}} .
\end{gathered}
$$

The estimate for $g^{\sharp}-\tilde{g}^{\sharp}$ can be derived in the same way. 
Bibliographic notes. Paraproducts were introduced in [4]. For a nice introduction see [1]. The commutator estimate Lemma 14 is from [14], but the proof here is new and the statement is slightly different. In [14], we require the additional assumption $\alpha \in(0,1)$ under which $C$ maps $\mathscr{C}^{\alpha} \times \mathscr{C}^{\beta} \times \mathscr{C}^{\gamma}$ to $\mathscr{C}^{\alpha+\beta+\gamma}$ and not only to $\mathscr{C}^{\beta+\gamma}$. Theorem 6 is from [14].

Theorem 7 is new, but it is implicitly used in [14]. The estimates presented here will only allow us to consider regular initial conditions. More general situations can be covered by working on spaces allowing for a singularity at 0 , such as

$$
\left\{f \in C\left((0, \infty), \mathscr{C}^{\alpha}\right): \sup _{t \in(0, T]}\left\|t^{-\gamma} f(t)\right\|_{\mathscr{C}^{\alpha}}<\infty \text { for all } T>0\right\}
$$

and similar for the temporal regularity. This is also done in [14].

Of course it is easily possible to replace the Laplacian by more general pseudo-differential operators. We only used two properties of $\Delta$ : the fact that $\Delta\left(f^{\prime} \prec U\right)-f^{\prime} \prec(\Delta U)$ is relatively regular, and that the semigroup generated by $\Delta$ has a sufficiently strong regularization effect. This is also true for the fractional Laplace operator and more generally for a wide range of pseudo-differential operators.

\subsection{Fixpoint}

Let us now give the details for the solution to PAM in the space of paracontrolled distributions. Assume that $F: \mathbb{R} \rightarrow \mathbb{R}$ is in $C_{b}^{1+\varepsilon}$ for some $\varepsilon>0$ such that $(2+\varepsilon) \gamma>2$.

Let $Y \in C \mathscr{C}^{\gamma}$ and let $u \in \mathscr{D}^{\gamma}(Y)$. We will see below how to choose $Y$, for the moment it is an arbitrary $C \mathscr{C}^{\gamma}$ function. From Theorem 6 we know that $F(u) \in \mathscr{D}^{\varepsilon \gamma}(Y)$ :

$$
\mathscr{D}^{\gamma}(Y) \stackrel{u \mapsto F(u)}{\longrightarrow} \mathscr{D}^{\varepsilon \gamma}(Y) .
$$

Assume now that $Y \circ \eta \in C \mathscr{C}^{2 \gamma-2}$ is given - note that for the regularity assumptions we made, $Y \circ \eta$ is not a continuous functional of $Y$ and $\eta$ but must be controlled using other means, say stochastic computations! Under this assumption, Theorem 5 applied with $u=Y, v=\eta$, and $\zeta=Y \circ \eta$ shows that for all $f \in \mathscr{D}^{\varepsilon \gamma}(Y)$ we have $f \eta=(f \eta)^{\sharp}+f \prec \eta$ with $(f \eta)^{\sharp} \in C \mathscr{C}^{2 \gamma-2}$ - it is here that we use $(2+\varepsilon) \gamma>2$. Integrating against the heat kernel and assuming that $u_{0} \in \mathscr{C}^{2 \gamma}$, we obtain from Theorem 7 (with $\left.u=\eta, f^{u}=f, f^{\sharp}=(f \eta)^{\sharp}\right)$ that the solution $\left(J(f \eta)(t)+P_{t} u_{0}\right)_{t \geqslant 0}$ to $\mathscr{L}\left(J(f \eta)+P . u_{0}\right)=f \eta, J(f \eta)(0)+P_{0} u_{0}=u_{0}$, is in $\mathscr{D}^{\gamma}(X)$, where $X$ solves $\mathscr{L} X=\eta$ and $X(0)=0$. In other words, we have a map

$$
\mathscr{D}^{\varepsilon \gamma}(Y) \stackrel{f \mapsto P . u_{0}+J(f \eta)}{\longrightarrow} \mathscr{D}^{\gamma}(X)
$$


and combining (5.13) and (5.14) we get

$$
\mathscr{D}^{\gamma}(Y) \stackrel{u \mapsto F(u)}{\longrightarrow} \mathscr{D}^{\varepsilon \gamma}(Y) \stackrel{F(u) \mapsto P . u_{0}+J(F(u) \eta)}{\longrightarrow} \mathscr{D}^{\gamma}(X),
$$

so that for all $T>0$ we can define

$$
\Gamma_{T}: \mathscr{D}_{T}^{\gamma}(Y) \rightarrow \mathscr{D}_{T}^{\gamma}(X), \quad \Gamma_{T}(u)=\left.\left(P . u_{0}+J(F(u) \eta)\right)\right|_{[0, T]} .
$$

To set up a Picard iteration domain and image space should coincide which means we should take $Y=X$. Refining the analysis, we obtain a scaling factor $T^{\delta}$ when estimating the $\mathscr{D}_{T}^{\gamma}(X)$-norm of $\Gamma_{T}(u)$. This allows us to show that for small $T>0$, the map $\Gamma_{T}$ leaves suitable balls in $\mathscr{D}_{T}^{\gamma}(X)$ invariant, and therefore we obtain the (local in time) existence of solutions to the equation under the assumption $X \circ \eta \in C \mathscr{C}^{2 \gamma-2}$.

To obtain uniqueness we need to suppose that $F \in C_{b}^{2+\varepsilon}$. In that case Theorem 6 gives the local Lipschitz continuity of the map $u \mapsto F(u)$ from $\mathscr{D}_{T}^{\gamma}(X)$ to $\mathscr{D}_{T}^{\varepsilon \gamma}(X)$, while Theorem 5 and Theorem 7 show that $f \mapsto u_{0}+J(f \eta)$ defines a Lipschitz continuous map from $\mathscr{D}_{T}^{\varepsilon \gamma}(X)$ to $\mathscr{D}_{T}^{\gamma}(X)$. Again we can obtain a scaling factor $T^{\delta}$, so that $\Gamma_{T}$ defines a contraction on a suitable ball of $\mathscr{D}_{T}^{\gamma}(X)$ for some small $T>0$.

Even better, $\Gamma_{T}$ not only depends locally Lipschitz continuously on $u$, but also on the extended data $\left(u_{0}, \eta, X \circ \eta\right)$, and therefore the solution to (5.1) depends locally Lipschitz continuously on $\left(u_{0}, \eta, X \circ \eta\right)$.

\subsection{Renormalization}

So far we argued under the assumption that $X \circ \eta$ exists and has a sufficient regularity. This should be understood via approximations as the existence of a sequence of smooth functions $\left(\eta_{n}\right)$ that converges to $\eta$, such that $\left(X_{n} \circ \eta_{n}\right)$ converges to $X \circ \eta$. However, as we will see below, this hypothesis is questionable and actually not satisfied at all in the problem we are interested in. More concretely, recall that we would like to take $\eta=\xi$ to be the two-dimensional space white noise. If then $\varphi$ is a Schwartz function on $\mathbb{R}^{2}$ and if $\varphi_{n}=n \varphi(n \cdot)$ and

$$
\eta_{n}(x)=\varphi_{n} * \xi(x)=\int_{\mathbb{R}^{2}} \varphi_{n}(x-y) \xi(y) \mathrm{d} y=\sum_{k \in \mathbb{Z}^{2}}\left\langle\xi, \varphi_{n}(x+2 \pi k-\cdot)\right\rangle,
$$

then we will see below that there exist constants $\left(c_{n}\right)$ with $\lim _{n} c_{n}=\infty$, such that $\left(X_{n} \circ \eta_{n}-c_{n}\right)$ converges in $C_{T} \mathscr{C}^{2 \gamma-2}$ for all $T>0$.

This is not a problem with our specific approximation. The homogenization setting shows that even for $\eta \rightarrow 0$ there are cases where the limiting equation is nontrivial. In the paracontrolled setting we have 
a continuous dependence of the solution on the data $(\eta, X \circ \eta)$, so this non-triviality of the limit can only mean that it is $X \circ \eta$ which does not converge to zero.

Another way to see that there is a problem is to consider the following representation of the resonant term: use $\mathscr{L} X=\eta$ to write

$$
\begin{aligned}
X \circ \eta & =X \circ \mathscr{L} X \\
& =\frac{1}{2} \mathscr{L}(X \circ X)+\partial_{x} X \circ \partial_{x} X \\
& =\left|\partial_{x} X\right|^{2}+\frac{1}{2} \mathscr{L}(X \circ X)-2 \partial_{x} X \prec \partial_{x} X .
\end{aligned}
$$

Integrating this equation over the torus and over $t \in[0, T]$, we get

$$
\begin{aligned}
\int_{0}^{T} \int_{\mathbb{T}^{2}} X \circ \eta \mathrm{d} x \mathrm{~d} t= & \int_{0}^{T} \int_{\mathbb{T}^{2}}\left|\partial_{x} X\right|^{2} \mathrm{~d} x \mathrm{~d} t+\frac{1}{2} \int_{0}^{T} \int_{\mathbb{T}^{2}} \mathscr{L}(X \circ X) \mathrm{d} x \\
& -2 \int_{0}^{T} \int_{\mathbb{T}^{2}}\left(\partial_{x} X \prec \partial_{x} X\right) \mathrm{d} x \mathrm{~d} t .
\end{aligned}
$$

Writing $\mathscr{L}=\partial_{t}-\Delta$ and using that $X(0)=0$ and $\int_{\mathbb{T}^{2}} \Delta \psi \mathrm{d} x=0$ for all $\psi$ (which can be seen using integration by parts and pulling the operator $\Delta$ on the constant function 1), we thus get

$$
\begin{aligned}
\int_{0}^{T} \int_{\mathbb{T}^{2}} X \circ \eta \mathrm{d} x \mathrm{~d} t= & \int_{0}^{T} \int_{\mathbb{T}^{2}}\left|\partial_{x} X\right|^{2} \mathrm{~d} x \mathrm{~d} t+\frac{1}{2} \int_{\mathbb{T}^{2}}(X(T) \circ X(T)) \mathrm{d} x \\
& -2 \int_{0}^{T} \int_{\mathbb{T}^{2}}\left(\partial_{x} X \prec \partial_{x} X\right) \mathrm{d} x \mathrm{~d} t .
\end{aligned}
$$

So if $X \circ \eta \in C_{T} \mathscr{C}^{2 \gamma-2}$ and $X \in C_{T} \mathscr{C}^{\gamma}$, then all the terms should be well defined and finite (the integral over $\mathbb{T}^{2}$ corresponds to testing a distribution against to constant test function 1). This would mean that $\int_{0}^{T} \int_{\mathbb{T}^{2}}\left|\partial_{x} X\right|^{2} \mathrm{~d} x \mathrm{~d} t<+\infty$, but on the other side a direct computation shows that

$$
\int_{\mathbb{T}^{2}}\left|\partial_{x} X(t, \cdot)\right|^{2} \mathrm{~d} x=+\infty
$$

for any $t>0$ almost surely if $\eta$ is the space white noise. Note also that the problematic term $\left|\partial_{x} X\right|^{2}$ is exactly the correction term appearing in the analysis of the linear homogenization problem in Section 4 .

In order to prove the convergence of the smooth solutions in general, we should introduce corrections to the equation to remove the divergent constant $c_{n}$. Let us see where the resonant product $X \circ \eta$ appears. We 
have

$$
(F(u) \eta)^{\sharp}=F(u) \succ \eta+(F(u))^{\sharp} \circ \eta+C\left((F(u))^{X}, X, \eta\right)+(F(u))^{X}(X \circ \eta) .
$$

Now $(F(u))^{X}=F^{\prime}(u) u^{X}$ by Theorem 6 , and if $u$ solves the equation $\mathscr{L} u=F(u) \eta=F(u) \prec \eta+(F(u) \eta)^{\sharp}$, then Theorem 7 with $u=\eta, X=U$ shows that $u^{X}=F(u)$. So we should really consider the renormalized equation

$$
\mathscr{L} u_{n}=F\left(u_{n}\right) \diamond \eta_{n}:=F\left(u_{n}\right) \xi_{n}-F^{\prime}\left(u_{n}\right) F\left(u_{n}\right) c_{n},
$$

where we recall that $\left(c_{n}\right)$ are the diverging constants for which $\left(X_{n} \circ \eta_{n}-\right.$ $c_{n}$ ) converges. In that case we have

$$
\begin{aligned}
\mathscr{L} u_{n}= & F\left(u_{n}\right) \prec \eta_{n}+F\left(u_{n}\right) \succ \eta_{n}+\left(F\left(u_{n}\right)\right)^{\sharp} \circ \eta_{n} \\
& +C\left(F^{\prime}\left(u_{n}\right) F\left(u_{n}\right), X_{n}, \eta_{n}\right)+F^{\prime}\left(u_{n}\right) F\left(u_{n}\right)\left(X_{n} \circ \eta_{n}-c_{n}\right),
\end{aligned}
$$

and now all the terms on the right hand side are under control and we can safely pass to the limit, for which we obtain the equation

$$
\mathscr{L} u=F(u) \diamond \eta:=(F(u) \diamond \eta)^{\sharp}+F(u) \prec \eta,
$$

where $(F(u) \diamond \eta)^{\sharp}$ is calculated using $X \diamond \eta=\lim _{n}\left(X_{n} \circ \eta_{n}-c_{n}\right)$ in the place of $X \circ \eta$ in (5.15). Formally, we also denote this product by

$$
F(u) \diamond \eta=F(u) \eta-F^{\prime}(u) F(u) \cdot \infty,
$$

so that the solution $u$ will satisfy

$$
\mathscr{L} u=F(u)-F^{\prime}(u) F(u) \cdot \infty .
$$

Note that the correction term has exactly the same form as the Itô/Stratonovich corrector for SDEs. For the reader familiar with rough paths this will not come as a surprise: Changing the iterated integrals of a rough path $B$ from some given $\int_{0}^{*} B_{s} \mathrm{~d} B_{s}$ to $\int_{0}^{*} B_{s} \mathrm{~d} B_{s}+\varphi$ introduces a correction term $+F^{\prime}(y) F(y) \partial_{t} \varphi$ in the ODE $\partial_{t} y=F(y) \partial_{t} B$. In our setting the resonant term takes the role of the iterated integrals, and since the structure of the ODE and GPAM is very similar changing the resonant term has a similar effect as changing the iterated integrals in the ODE example.

Remark 9. The convergence properties of $\left(X_{n} \circ \eta_{n}\right)$ are in stark contrast to the ODE setting: if we consider the equation $\partial_{t} u=F(u) \zeta$ rather than PAM, then we should replace $X$ by $Z$ with $\partial_{t} Z=\zeta$. But then we have in one dimension $Z \circ \zeta=1 / 2 \partial_{t}(Z \circ Z)$, so that the convergence of $\left(Z_{n} \circ \zeta_{n}\right)$ to $Z \circ \zeta$ comes for free with the convergence of $\left(Z_{n}\right)$ to $Z$. Indeed, $\partial_{t}$ is a 
bounded linear operator from $\mathscr{C}^{\gamma}$ to $\mathscr{C}^{\gamma-1}$ whenever $\gamma \in \mathbb{R}$, and $Z \mapsto Z \circ Z$ is continuous from $\mathscr{C}^{\gamma}$ to $\mathscr{C}^{2 \gamma}$ whenever $\gamma>0$. So if $\left(Z_{n}\right)$ converges to $Z$ in a Hölder space of positive regularity, then $\left(\partial_{t}\left(Z_{n} \circ Z_{n}\right)\right)$ converges to $\partial_{t}(Z \circ Z)$. This specific representation of $Z \circ \zeta$ comes from the Leibniz rule for $\partial_{t}$ and it is the reason why rough path theory is trivial in one dimension, at least as long as one considers those rough paths which are limit of smooth paths. Of course, the argument breaks down as soon as $Z$ has at least two components. As we have discussed, for the second order differential operator $\mathscr{L}$ we have different rules and obtain

$$
(X \circ \eta)=(X \circ \mathscr{L} X)=\frac{1}{2} \mathscr{L}(X \circ X)+\left(\partial_{x} X \circ \partial_{x} X\right),
$$

so that in our setting the nontrivial term is $\partial_{x} X \circ \partial_{x} X$.

These considerations lead naturally to the following definition.

Definition 6. (PAM-enhancement) Let $\gamma \in(2 / 3,1)$ and let

$$
\mathcal{X}_{\text {pam }}^{\gamma} \subseteq \mathscr{C}^{\gamma-2} \times C \mathscr{C}^{2 \gamma-2}
$$

be the closure of the image of the map

$$
\Theta_{\text {pam }}: C^{\infty} \times C([0, \infty), \mathbb{R}) \rightarrow \mathcal{X}_{\text {pam }}^{\gamma},
$$

given by

$$
\Theta_{\operatorname{pam}}(\theta, f)=(\theta, \Phi \diamond \theta):=(\theta, \Phi \circ \theta-f),
$$

where $\Phi=J \theta$, that is $\mathscr{L} \Phi=\theta$ and $\Phi(0)=0$. We will call $\Theta_{\text {pam }}(\theta, f)$ the renormalized PAM-enhancement of the driving distribution $\theta$. For $T>0$ we define $\mathcal{X}_{\mathrm{pam}}^{\gamma}(T)=\left.\mathcal{X}_{\mathrm{pam}}^{\gamma}\right|_{[0, T]}$ and we write $\|\mathbb{X}\|_{\mathcal{X}_{\mathrm{pam}}^{\gamma}(T)}$ for the norm of $\mathbb{X} \in \mathcal{X}_{\text {pam }}^{\gamma}(T)$ in the Banach space $\mathscr{C}^{\gamma-2} \times C_{T} \mathscr{C}^{2 \gamma-2}$. Moreover, we define the distance $d_{\mathcal{X}_{\mathrm{pam}}^{\gamma}(T)}(\mathbb{X}, \tilde{\mathbb{X}})=\|\mathbb{X}-\tilde{\mathbb{X}}\|_{\mathcal{X}_{\mathrm{pam}}^{\gamma}(T)}$.

Remark 10. In the homogenization example of Section 4 we would take $\theta=V_{\varepsilon}$ and $\Phi=X_{\varepsilon}$.

Remark 11. It would be more elegant to renormalize $\Phi \circ \theta$ with a constant and not with a time-dependent function, as we discussed above. Indeed this is possible, see Chapter 5 of [14]. But since here we chose $\Phi(0)=0$, we have $\Phi(0) \circ \theta=0$ and therefore $\left(\Phi_{n}(0) \circ \theta_{n}-c_{n}\right)$ diverges for any diverging sequence of constants $\left(c_{n}\right)$. A simple way of avoiding this problem is to consider the stationary version $\tilde{\Phi}$ given by

$$
\tilde{\Phi}(x)=\int_{0}^{\infty} P_{t} \Pi_{\neq 0} \theta(x) \mathrm{d} t,
$$

where $\Pi_{\neq 0}$ denotes the projection on the non-zero Fourier modes, $\Pi_{\neq 0} u=$ $u-(2 \pi)^{-d / 2} \hat{u}(0)$. But then $\tilde{\Phi}$ does not depend on time and in particular 
$\tilde{\Phi}(0) \neq 0$, so that we have to consider irregular initial conditions in the paracontrolled approach which complicates the presentation. Alternatively, we could observe that in the white noise case there exist constants $\left(c_{n}\right)$ so that $\left(X_{n}(t) \circ \xi_{n}-c_{n}\right)$ converges for all $t>0$, and while the limit $(X(t) \diamond \xi)$ diverges as $t \rightarrow 0$, it can be integrated against the heat kernel. Again, this would complicate the presentation and here we choose the simple (and cheap) solution of taking a time-dependent renormalization.

Theorem 8. Let $\gamma \in(2 / 3,1)$ and $\varepsilon>0$ be such that $(2+\varepsilon) \gamma>2$. Let $\mathbb{X}=(\eta, X \diamond \eta) \in \mathcal{X}_{\text {pam }}^{\gamma}, F \in C_{b}^{2+\varepsilon}$, and $u_{0} \in \mathscr{C}^{2 \gamma}$. Then there exists a unique solution $u \in \mathscr{D}^{\gamma}(X)$ to the equation

$$
\mathscr{L} u=F(u) \diamond \eta, \quad u(0)=u_{0},
$$

up to the (possibly finite) explosion time $\tau=\tau(u)=\inf \left\{t \geqslant 0:\|u\|_{\mathscr{D}_{t}^{\gamma}}=\right.$ $\infty\}>0$.

Moreover, $u$ depends on $\left(u_{0}, \mathbb{X}\right) \in \mathscr{C}^{2 \gamma} \times \mathcal{X}_{\text {pam }}^{\gamma}$ in a locally Lipschitz continuous way: if $M, T>0$ are such that for all $\left(u_{0}, \mathbb{X}\right)$ with $\left\|u_{0}\right\|_{2 \gamma} \vee$ $\|\mathbb{X}\|_{\mathcal{X}_{\mathrm{pam}}^{\gamma}(T)} \leqslant M$, the solution $u$ to the equation driven by $\left(u_{0}, \mathbb{X}\right)$ satisfies $\tau(u)>T$, and if $\left(\tilde{u}_{0}, \tilde{\mathbb{X}}\right)$ is another set of data bounded in the above sense by $M$, then there exists $C(F, M)>0$ for which

$$
d_{\mathscr{D}_{T}^{\gamma}}(u, \tilde{u}) \leqslant C(F, M)\left(\left\|u_{0}-\tilde{u}_{0}\right\|_{2 \gamma}+d_{\mathcal{X}_{\mathrm{pam}}^{\gamma}(T)}(\mathbb{X}, \tilde{\mathbb{X}})\right) .
$$

Proof. We only have to turn the formal discussion of Section 5.4 into rigorous mathematics. The small factor $T^{\delta}$ on page 71 is obtained from a scaling argument and while this does not require any new insights it is somewhat lengthy and we refer to $[14,16]$ for details.

Let us just indicate how to iterate the construction to obtain the existence of solutions up to the explosion time $\tau$. Let us assume that we constructed the paracontrolled solution $\left(u, u^{X}\right.$ ) (with $u^{X}=F(u)$ ) on $\left[0, T_{0}\right]$ for some $T_{0}>0$. Now we no longer have $X\left(T_{0}\right)=0$, and also the initial condition $u\left(T_{0}\right)$ is no longer in $\mathscr{C}^{2 \gamma}$. But we only used $X(0)=0$ to see that the initial condition for $u^{\sharp}$ is $u^{\sharp}(0)=u_{0}$, and we only used $u_{0} \in \mathscr{C}^{2 \gamma}$ to obtain a $\mathscr{C}^{2 \gamma}$ initial condition for $u^{\sharp}$. On the next interval, the initial condition for $u^{\sharp}$ is $u^{\sharp}\left(T_{0}\right)=u\left(T_{0}\right)-F\left(u\left(T_{0}\right)\right) \prec X\left(T_{0}\right)$ which is in $\mathscr{C}^{2 \gamma}$ by construction, since we already know that $u^{\sharp} \in C\left(\left[0, T_{0}\right], \mathscr{C}^{2 \gamma}\right)$.

As for the continuity in $\left(u_{0}, \mathbb{X}\right)$, let $\left(\tilde{u}_{0}, \tilde{\mathbb{X}}\right)$ be another set of data also bounded by $M$. Then the solutions $u$ and $\tilde{u}$ both are bounded in $\mathscr{D}_{T}^{\gamma}$ by some constant $c=c(F, M)>0$. So by the continuity properties of the paracontrolled product (and the other operations involved), we can estimate

$$
d_{\mathscr{D}_{T}^{\gamma}}(u, \tilde{u}) \leqslant P(c)\left(\left\|u_{0}-\tilde{u}_{0}\right\|_{2 \gamma}+d_{\mathcal{X}_{\text {pam }}^{\gamma}(T)}(\mathbb{X}, \tilde{\mathbb{X}})+T^{\delta} d_{\mathscr{D}_{T}^{\gamma}}(u, \tilde{u})\right)
$$

for a polynomial $P$. The local Lipschitz continuity on $[0, T]$ immediately follows if we choose $T>0$ small enough. This can be iterated to obtain the local Lipschitz continuity on "macroscopic" intervals. 
Remark 12. For the local in time existence it is not necessary to assume $F \in C_{b}^{2+\varepsilon}$. It suffices to have $F \in C^{2+\varepsilon}$. This can be seen by considering a ball containing $u_{0}(x)$ for all $x \in \mathbb{T}^{d}$, a function $\tilde{F} \in C_{b}^{2+\varepsilon}$ which coincides with $F$ on this ball, and by stopping $u$ upon exiting the ball.

In the linear case $F(u)=u$ we have global in time solutions: in general we only get local in time solutions because we pick up a superlinear (polynomial) estimate when applying the paralinearization result Theorem 6. This step is not necessary if $F$ is linear, and all the other estimates are linear in $u$.

\subsection{Construction of the extended data}

In order to apply Theorem 8 to equation (5.1) with white noise perturbation, it remains to show that if $\xi$ is a spatial white noise on $\mathbb{T}^{2}$, then $(\xi, X \diamond \xi)$ defines an element of $\mathcal{X}_{\mathrm{pam}}^{\gamma}$ whenever $\gamma \in(2 / 3,1)$. In other words, we need to construct $X \diamond \xi$ and control its regularity.

Since $P_{t} \xi$ is a smooth function for every $t>0$, the resonant term $P_{t} \xi \circ \xi$ is a smooth function, and therefore we could formally set $X(t) \circ \xi=$ $\int_{0}^{t}\left(P_{s} \xi \circ \xi\right) \mathrm{d} s$. But we will see that this expression does not make sense.

Recall that $(\hat{\xi}(k))_{k \in \mathbb{Z}^{2}}$ is a complex valued, centered Gaussian process with covariance

$$
\mathbb{E}\left[\hat{\xi}(k) \hat{\xi}\left(k^{\prime}\right)\right]=\delta_{k+k^{\prime}=0},
$$

and such that $\hat{\xi}(k)^{*}=\hat{\xi}(-k)$.

Lemma 19. For any $x \in \mathbb{T}^{2}$ and $t>0$ we have $g_{t}=\mathbb{E}\left[\left(P_{t} \xi\right)(x) \xi(x)\right]=\mathbb{E}\left[\left(P_{t} \xi \circ \xi\right)(x)\right]=\mathbb{E}\left[\Delta_{-1}\left(P_{t} \xi \circ \xi\right)(x)\right]=(2 \pi)^{-2} \sum_{k \in \mathbb{Z}^{2}} e^{-t|k|^{2}}$.

In particular, $g_{t}$ does not depend on the partition of unity used to define the $\circ$ operator, and $\int_{0}^{t} g_{s} \mathrm{~d} s=\infty$ for all $t>0$.

Proof. Let $x \in \mathbb{T}^{2}, t>0$, and $\ell \geq-1$. Then

$$
\mathbb{E}\left[\Delta_{\ell}\left(P_{t} \xi \circ \xi\right)(x)\right]=\sum_{|i-j| \leqslant 1} \mathbb{E}\left[\Delta_{\ell}\left(\Delta_{i}\left(P_{t} \xi\right) \Delta_{j} \xi\right)(x)\right],
$$

where exchanging summation and expectation is justified because it can be easily verified that the partial sums of $\Delta_{\ell}\left(P_{t} \xi \circ \xi\right)(x)$ are uniformly $L^{p}$-bounded for any $p \geq 1$. Now $P_{t}=e^{-t|\cdot|^{2}}(\mathrm{D})$, and therefore we get 
from (5.18)

$$
\begin{aligned}
& \mathbb{E}\left[\Delta_{\ell}\left(\Delta_{i}\left(P_{t} \xi\right) \Delta_{j} \xi\right)(x)\right] \\
= & (2 \pi)^{-1} \sum_{k, k^{\prime} \in \mathbb{Z}^{2}} e_{k+k^{\prime}}^{*}(x) \rho_{\ell}\left(k+k^{\prime}\right) \rho_{i}(k) e^{-t|k|^{2}} \rho_{j}\left(k^{\prime}\right) \mathbb{E}\left[\hat{\xi}(k) \hat{\xi}\left(k^{\prime}\right)\right] \\
= & (2 \pi)^{-2} \sum_{k \in \mathbb{Z}^{2}} \rho_{\ell}(0) \rho_{i}(k) e^{-t|k|^{2}} \rho_{j}(k)=\delta_{\ell=-1}(2 \pi)^{-2} \sum_{k \in \mathbb{Z}^{2}} \rho_{i}(k) \rho_{j}(k) e^{-t|k|^{2}} .
\end{aligned}
$$

For $|i-j|>1$ we have $\rho_{i}(k) \rho_{j}(k)=0$ and therefore

$$
\begin{aligned}
g_{t} & =\mathbb{E}\left[\left(P_{t} \xi \circ \xi\right)(x)\right] \\
& =\mathbb{E}\left[\left(P_{t} \xi\right)(x) \xi(x)\right] \\
& =(2 \pi)^{-2} \sum_{k \in \mathbb{Z}^{2}} \sum_{i, j} \rho_{i}(k) \rho_{j}(k) e^{-t|k|^{2}} \\
& =(2 \pi)^{-2} \sum_{k \in \mathbb{Z}^{2}} e^{-t|k|^{2}}
\end{aligned}
$$

while $\left.\mathbb{E}\left[\left(P_{t} \xi \circ \xi\right)(x)-\Delta_{-1}\left(P_{t} \xi \circ \xi\right)\right)(x)\right]=0$.

Exercise 12. Let $\varphi$ be a Schwartz function on $\mathbb{R}^{2}$ and set

$$
\begin{aligned}
\xi_{n}(x) & =\left(\left(n^{2} \varphi(n \cdot)\right) * \xi\right)(x) \\
& =\int_{\mathbb{R}^{2}} n^{2} \varphi(n(x-y)) \xi(y) \mathrm{d} y \\
& =\sum_{k \in \mathbb{Z}^{2}}\left\langle\xi, n^{2} \varphi(n(x+2 \pi k-\cdot))\right\rangle
\end{aligned}
$$

for $x \in \mathbb{T}^{2}$. Write $\mathscr{F}_{\mathbb{R}^{2}} \varphi(z)=\int_{\mathbb{R}^{2}} e^{-i\langle z, x\rangle} \varphi(x) \mathrm{d} x$. Show that $\mathbb{E}\left[\left(P_{t} \xi_{n} \circ \xi_{n}\right)(x)\right]=\mathbb{E}\left[\Delta_{-1}\left(P_{t} \xi_{n} \circ \xi_{n}\right)(x)\right]=(2 \pi)^{-2} \sum_{k \in \mathbb{Z}^{2}} e^{-t|k|^{2}}\left|\mathscr{F}_{\mathbb{R}^{2}} \varphi(k / n)\right|^{2}$.

Hint: Use Poisson summation.

The diverging time integral motivates us to study the renormalized product $X \circ \xi-\int_{0}^{\cdot} g_{s} \mathrm{~d} s$, where $\int_{0}^{\cdot} g_{s} \mathrm{~d} s$ is an "infinite function":

Lemma 20. Set

$$
(X \diamond \xi)(t)=\int_{0}^{t}\left(P_{s} \xi \circ \xi-g_{s}\right) \mathrm{d} s .
$$

Then $\mathbb{E}\left[\|X \diamond \xi\|_{C_{T} \mathscr{C}^{2 \gamma-2}\left(\mathbb{T}^{2}\right)}^{p}\right]<\infty$ for all $\gamma<1, p \geq 1, T>0$. Moreover, if $\varphi$ is a Schwartz function on $\mathbb{R}^{2}$ with $\int \varphi(x) \mathrm{d} x=1$, if $\xi_{n}=\varphi_{n} * \xi$ with $\varphi_{n}=n^{2} \varphi(n \cdot)$ for $n \in \mathbb{N}$, and $X_{n}(t)=\int_{0}^{t} P_{s} \xi_{n} \mathrm{~d} s$, then

$$
\lim _{n \rightarrow \infty} \mathbb{E}\left[\left\|X \diamond \xi-\left(X_{n} \circ \xi_{n}-f_{n}\right)\right\|_{C_{T} \mathscr{C}^{2 \gamma-2}\left(\mathbb{T}^{2}\right)}^{p}\right]=0
$$


for all $p \geq 1$, where for all $x \in \mathbb{T}^{2}$

$$
\begin{aligned}
f_{n}(t) & =\mathbb{E}\left[X_{n}(t, x) \xi_{n}(x)\right]=\mathbb{E}\left[\left(X_{n}(t) \circ \xi_{n}\right)(x)\right] \\
& =(2 \pi)^{-2} \sum_{k \in \mathbb{Z}^{2} \backslash\{0\}} \frac{\left|\mathscr{F}_{\mathbb{R}^{2}} \varphi(k / n)\right|^{2}}{|k|^{2}}\left(1-e^{-t|k|^{2}}\right)+(2 \pi)^{-2} t .
\end{aligned}
$$

Proof. To lighten the notation, we will only show that $\mathbb{E}\left[\|X \diamond \xi\|_{C_{T} \mathscr{C}^{2 \gamma-2}}^{p}\right]<\infty$. The convergence of $\left(X_{n} \circ \xi_{n}-f_{n}\right)$ to $X \diamond \xi$ is shown by applying dominated convergence, and we leave it as an exercise. Let $t>0$ and define $\Xi_{t}=P_{t} \xi \circ \xi-g_{t}$. Let us start by estimating $\mathbb{E}\left[\left|\Delta_{\ell} \Xi_{t}(x)\right|^{2}\right]$ for $\ell \geqslant-1$ and $x \in \mathbb{T}^{2}$. Lemma 19 yields $\Delta_{\ell} g_{t}=0=\mathbb{E}\left[\Delta_{\ell}\left(P_{t} \xi \circ \xi\right)(x)\right]$ for $\ell \geq 0$ and $x \in \mathbb{T}^{2}$, and $\Delta_{-1} g_{t}=g_{t}=$ $\mathbb{E}\left[\Delta_{-1}\left(P_{t} \xi \circ \xi\right)(x)\right]$, so that $\mathbb{E}\left[\left|\Delta_{\ell} \Xi_{t}(x)\right|^{2}\right]=\operatorname{Var}\left(\Delta_{\ell}\left(P_{t} \xi \circ \xi\right)(x)\right)$. But

$$
\begin{aligned}
& \Delta_{\ell}\left(P_{t} \xi \circ \xi\right)(x) \\
= & \left.\sum_{k \in \mathbb{Z}^{2}} e_{k}^{*}(x) \rho_{\ell}(k) \mathscr{F}\left(P_{t} \xi \circ \xi\right)\right)(k) \\
= & (2 \pi)^{-1} \sum_{k_{1}, k_{2} \in \mathbb{Z}^{2}} \sum_{|i-j| \leqslant 1} e_{k_{1}+k_{2}}^{*}(x) \rho_{\ell}\left(k_{1}+k_{2}\right) \rho_{i}\left(k_{1}\right) e^{-t\left|k_{1}\right|^{2}} \hat{\xi}\left(k_{1}\right) \rho_{j}\left(k_{2}\right) \hat{\xi}\left(k_{2}\right),
\end{aligned}
$$

and therefore

$$
\begin{aligned}
& \operatorname{Var}\left(\Delta_{\ell}\left(P_{t} \xi \circ \xi\right)(x)\right) \\
= & (2 \pi)^{-2} \sum_{k_{1}, k_{2}} \sum_{k_{1}^{\prime}, k_{2}^{\prime}} \sum_{|i-j| \leqslant 1} \sum_{\left|i^{\prime}-j^{\prime}\right| \leqslant 1} e_{k_{1}+k_{2}}^{*}(x) \rho_{\ell}\left(k_{1}+k_{2}\right) \rho_{i}\left(k_{1}\right) e^{-t\left|k_{1}\right|^{2}} \rho_{j}\left(k_{2}\right) \\
& \times e_{k_{1}^{\prime}+k_{2}^{\prime}}^{*}(x) \rho_{\ell}\left(k_{1}^{\prime}+k_{2}^{\prime}\right) \rho_{i^{\prime}}\left(k_{1}^{\prime}\right) e^{-t\left|k_{1}^{\prime}\right|^{2}} \rho_{j^{\prime}}\left(k_{2}^{\prime}\right) \operatorname{Cov}\left(\hat{\xi}\left(k_{1}\right) \hat{\xi}\left(k_{2}\right), \hat{\xi}\left(k_{1}^{\prime}\right) \hat{\xi}\left(k_{2}^{\prime}\right)\right),
\end{aligned}
$$

where exchanging summation and expectation can be justified a posteriori by the uniform $L^{p}$-boundedness of the partial sums. Now Wick's theorem ([22], Theorem 1.28) gives

$$
\begin{aligned}
& \operatorname{Cov}\left(\hat{\xi}\left(k_{1}\right) \hat{\xi}\left(k_{2}\right), \hat{\xi}\left(k_{1}^{\prime}\right) \hat{\xi}\left(k_{2}^{\prime}\right)\right) \\
= & \mathbb{E}\left[\hat{\xi}\left(k_{1}\right) \hat{\xi}\left(k_{2}\right) \hat{\xi}\left(k_{1}^{\prime}\right) \hat{\xi}\left(k_{2}^{\prime}\right)\right]-\mathbb{E}\left[\hat{\xi}\left(k_{1}\right) \hat{\xi}\left(k_{2}\right)\right] \mathbb{E}\left[\hat{\xi}\left(k_{1}^{\prime}\right) \hat{\xi}\left(k_{2}^{\prime}\right)\right] \\
= & \mathbb{E}\left[\hat{\xi}\left(k_{1}\right) \hat{\xi}\left(k_{2}\right)\right] \mathbb{E}\left[\hat{\xi}\left(k_{1}^{\prime}\right) \hat{\xi}\left(k_{2}^{\prime}\right)\right]+\mathbb{E}\left[\hat{\xi}\left(k_{1}\right) \hat{\xi}\left(k_{1}^{\prime}\right)\right] \mathbb{E}\left[\hat{\xi}\left(k_{2}\right) \hat{\xi}\left(k_{2}^{\prime}\right)\right] \\
& +\mathbb{E}\left[\hat{\xi}\left(k_{1}\right) \hat{\xi}\left(k_{2}^{\prime}\right)\right] \mathbb{E}\left[\hat{\xi}\left(k_{2}\right) \hat{\xi}\left(k_{1}^{\prime}\right)\right]-\mathbb{E}\left[\hat{\xi}\left(k_{1}\right) \hat{\xi}\left(k_{2}\right)\right] \mathbb{E}\left[\hat{\xi}\left(k_{1}^{\prime}\right) \hat{\xi}\left(k_{2}^{\prime}\right)\right] \\
= & \left(\delta_{k_{1}+k_{1}^{\prime}=0} \delta_{k_{2}+k_{2}^{\prime}=0}+\delta_{k_{1}+k_{2}^{\prime}=0} \delta_{k_{2}+k_{1}^{\prime}=0}\right),
\end{aligned}
$$

which leads to

$$
\begin{aligned}
\operatorname{Var}\left(\Delta_{\ell}\left(P_{t} \xi \circ \xi\right)(x)\right)= & (2 \pi)^{-4} \sum_{k_{1}, k_{2}} \sum_{|i-j| \leqslant 1} \sum_{\left|i^{\prime}-j^{\prime}\right| \leqslant 1} \mathbb{I}_{\ell \lesssim i} \mathbb{I}_{\ell i^{\prime}} \rho_{\ell}^{2}\left(k_{1}+k_{2}\right) \rho_{i}\left(k_{1}\right) \rho_{j}\left(k_{2}\right) \\
& \times\left[\rho_{i^{\prime}}\left(k_{1}\right) \rho_{j^{\prime}}\left(k_{2}\right) e^{-2 t\left|k_{1}\right|^{2}}+\rho_{i^{\prime}}\left(k_{2}\right) \rho_{j^{\prime}}\left(k_{1}\right) e^{-t\left|k_{1}\right|^{2}-t\left|k_{2}\right|^{2}}\right] .
\end{aligned}
$$


Observe that there exists $c>0$ such that $e^{-2 t|k|^{2}} \lesssim e^{-t c 2^{2 i}}$ for all $k \in \operatorname{supp}\left(\rho_{i}\right) \cup \operatorname{supp}\left(\rho_{j}\right)$ with $i, j \geq-1$ and $|i-j| \leqslant 1$. Thus

$$
\begin{aligned}
& \operatorname{Var}\left(\Delta_{\ell}\left(P_{t} \xi \circ \xi\right)(x)\right) \\
\lesssim & \sum_{i, j, i^{\prime}, j^{\prime}} \mathbb{I}_{\ell \lesssim i} \mathbb{I}_{i \sim j \sim i^{\prime} \sim j^{\prime}} \sum_{k_{1}, k_{2}} \mathbb{I}_{\operatorname{supp}\left(\rho_{\ell}\right)}\left(k_{1}+k_{2}\right) \mathbb{I}_{\operatorname{supp}\left(\rho_{i}\right)}\left(k_{1}\right) \mathbb{I}_{\operatorname{supp}\left(\rho_{j}\right)}\left(k_{2}\right) e^{-2 t c 2^{2 i}} \\
\lesssim & \sum_{i: i \gtrsim \ell} 2^{2 i} 2^{2 \ell} e^{-t c 2^{2 i}} \lesssim \frac{2^{2 \ell}}{t} \sum_{i: i \gtrsim \ell} e^{-t c^{\prime} 2^{2 i}} \lesssim \frac{2^{2 \ell}}{t} e^{-t c^{\prime} 2^{2 \ell}},
\end{aligned}
$$

where in the third step we used that $t 2^{2 i} \lesssim e^{t\left(c-c^{\prime}\right) 2^{2 i}}$ for all $0<c^{\prime}<c$.

Consider now $X \diamond \xi(t)=\int_{0}^{t} \Xi_{s} \mathrm{~d} s$. We have for all $0 \leqslant s<t$

$$
\begin{aligned}
& \mathbb{E}\left[\|X \diamond \xi(t)-X \diamond \xi(s)\|_{B_{2 p, 2 p}^{2 \gamma-2}}^{2 p}\right] \\
= & \sum_{\ell} 2^{2 p \ell(2 \gamma-2)} \int_{\mathbb{T}^{2}} \mathbb{E}\left[\left|\Delta_{\ell}(X \diamond \xi(t)-X \diamond \xi(s))(x)\right|^{2 p}\right] \mathrm{d} x .
\end{aligned}
$$

Since the random variable $\Delta_{\ell}(X \diamond \xi(t)-X \diamond \xi(s))(x)$ lives in the second non-homogeneous chaos generated by the Gaussian white noise $\xi$, we may use Gaussian hypercontractivity ([22], Theorem 3.50) to bound

$$
\begin{aligned}
\mathbb{E}\left[\left|\Delta_{\ell}(X \diamond \xi(t)-X \diamond \xi(s))(x)\right|^{2 p}\right] & \lesssim \mathbb{E}\left[\left|\Delta_{\ell}(X \diamond \xi(t)-X \diamond \xi(s))(x)\right|\right]^{2 p} \\
& \leqslant\left(\int_{s}^{t} \mathbb{E}\left[\left|\Delta_{\ell} \Xi_{r}(x)\right|\right] \mathrm{d} r\right)^{2 p} .
\end{aligned}
$$

But we just showed that

$$
\begin{aligned}
\mathbb{E}\left[\left|\Delta_{\ell} \Xi_{r}(x)\right|\right] & \leqslant \mathbb{E}\left[\left|\Delta_{\ell} \Xi_{r}(x)\right|^{2}\right]^{1 / 2}=\left(\operatorname{Var}\left(\Delta_{\ell}\left(P_{r} \xi \circ \xi\right)(x)\right)\right)^{1 / 2} \\
& \lesssim r^{-1 / 2} 2^{\ell} e^{-\frac{1}{2} r c^{\prime} 2^{2 \ell}}=r^{-1 / 2} 2^{\ell} e^{-r c^{\prime \prime} 2^{2 \ell}}
\end{aligned}
$$

for $c^{\prime \prime}=c^{\prime} / 2>0$, and therefore

$$
\begin{aligned}
& \left(\mathbb{E}\left[\|X \diamond \xi(t)-X \diamond \xi(s)\|_{B_{2 p, 2 p}^{2 \gamma-2}}^{2 p}\right]\right)^{1 / 2 p} \\
\lesssim & \left(\sum_{\ell}\left(2^{\ell(2 \gamma-2)} \int_{s}^{t} r^{-1 / 2} 2^{\ell} e^{-r c^{\prime \prime} 2^{2 \ell}} \mathrm{d} r\right)^{2 p}\right)^{1 / 2 p} \\
\leqslant & \sum_{\ell} 2^{\ell(2 \gamma-1)} \int_{s}^{t} r^{-1 / 2} e^{-r c^{\prime \prime} 2^{2 \ell}} \mathrm{d} r \\
\lesssim & \int_{s}^{t} r^{-1 / 2} \int_{-1}^{\infty}\left(2^{x}\right)^{2 \gamma-1} e^{-r c^{\prime \prime} 2^{2 x}} \mathrm{~d} x \mathrm{~d} r
\end{aligned}
$$

The change of variable $y=\sqrt{r} 2^{x}$ leads to

$$
\left(\mathbb{E}\left[\|X \diamond \xi(t)-X \diamond \xi(s)\|_{B_{2 p}^{2 \gamma, 2 p}}^{2 p}\right]\right)^{1 / 2 p} \lesssim \int_{s}^{t} r^{-1 / 2} r^{-(2 \gamma-1) / 2} \int_{0}^{\infty} y^{2 \gamma-2} e^{-c^{\prime \prime} y^{2}} \mathrm{~d} y \mathrm{~d} r .
$$


For $\gamma>1 / 2$, the integral in $y$ is finite and we end up with

$$
\left(\mathbb{E}\left[\|X \diamond \xi(t)-X \diamond \xi(s)\|_{B_{2 p, 2 p}^{2 \gamma-2}}^{2 p}\right]\right)^{1 / 2 p} \lesssim \int_{s}^{t} r^{-\gamma} \mathrm{d} r \lesssim|t-s|^{1-\gamma}
$$

provided that $\gamma \in(1 / 2,1)$. So for large enough $p$ we can use Kolmogorov's continuity criterion to deduce that (modulo taking a modification of $X \diamond \xi$ ) we have $\mathbb{E}\left[\|X \diamond \xi\|_{C_{T} B_{2 p, 2 p}^{2 \gamma-2}}^{2 p}\right]<\infty$ for all $T>0$. Since this holds for all $\gamma<1$, the claim now follows from the Besov embedding theorem, Lemma 8 .

Combining Theorem 8 and Lemma 20, we are finally able to solve (5.1) driven by a space white noise.

Corollary 2. Let $\varepsilon>0$ and let $F \in C_{b}^{2+\varepsilon}$ and assume that $u_{0}$ is a random variable that almost surely takes its values in $\mathscr{C}^{2 \gamma}$ for some $\gamma \in(2 / 3,1)$ with $(2+\varepsilon) \gamma>2$. Let $\xi$ be a spatial white noise on $\mathbb{T}^{2}$. Then there exists a unique solution $u$ to

$$
\mathscr{L} u=F(u) \diamond \xi, \quad u(0)=u_{0},
$$

up to the (possibly finite) explosion time $\tau=\tau(u)=\inf \left\{t \geqslant 0:\|u\|_{\mathscr{D}_{t}^{\gamma}}=\right.$ $\infty\}$ which is almost surely strictly positive.

If $\left(\varphi_{n}\right)$ and $\left(\xi_{n}\right)$ are as described in Lemma 20, and if $\left(u_{0, n}\right)$ converges in probability in $\mathscr{C}^{2 \gamma}$ to $u_{0}$, then $u$ is the limit in probability of the solutions $u_{n}$ to

$$
\mathscr{L} u_{n}=F\left(u_{n}\right) \diamond \xi_{n}, \quad u_{n}(0)=u_{0, n} .
$$

Remark 13. We even have a stronger result: We can fix a null set outside of which $X \diamond \xi$ is regular enough, and once we dispose of that null set we can solve all equations for any regular enough $u_{0}$ and $F$ simultaneously, without ever having to worry about null sets again. This is for example interesting when studying stochastic flows or when studying equations with random $u_{0}$ and $F$.

The pathwise continuous dependence on the signal is also powerful in several other applications, for example support theorems and large deviations. For examples in the theory of rough paths see [9]. 


\section{Chapter 6}

\section{The stochastic Burgers equation}

Let us now return to the stochastic Burgers equation SBE

$$
\mathscr{L} u=\partial_{x} u^{2}+\partial_{x} \xi, \quad u(0)=u_{0},
$$

where $u:[0, \infty) \times \mathbb{T} \rightarrow \mathbb{R}, \xi$ is a space-time white noise, and $\partial_{x}$ denotes the spatial derivative. As we argued before, the solution $u$ cannot be expected to behave better than the Ornstein-Uhlenbeck process $X$, the solution of the linear equation $\mathscr{L} X=\partial_{x} \xi$, and as we saw in Section $2 X(t)$ is for all $t>0$ a smooth function of the space variable plus a space white noise. By Exercise 11, the white noise in dimension 1 has regularity $\mathscr{C}^{-1 / 2-}$. Thus $X \in C \mathscr{C}^{-1 / 2-}$, and in particular $u^{2}$ is the square of a distribution and a priori not well defined.

What raises some hope is that in Lemma 2 we were able to show that $\partial_{x} X^{2}$ exists as a space-time distribution. So as in the previous examples there are stochastic cancellations going into $\partial_{x} X^{2}$. The energy solution approach was designed to take those cancellations into account in the full solution $u$, but while it allowed us to work under rather weak assumptions which easily gave us existence of solutions, it did not give us sufficient control to have uniqueness of solutions. On the other side, a suitable paracontrolled ansatz for the solution $u$ will allow us to transfer the cancellation properties of $X$ to $u$ and it will allow us to construct $\partial_{x} u^{2}$ as a continuous bilinear map, from where existence and uniqueness of solutions easily follows. 


\subsection{Structure of the solution}

In this discussion we consider the case of zero initial condition and smooth noise $\xi$, and we analyze the structure of the solution. Let us expand $u$ around the Ornstein-Uhlenbeck process $X$ with $\mathscr{L} X=\partial_{x} \xi, X(0)=0$. Setting $u=X+u \geqslant 1$, we have

$$
\mathscr{L} u^{\geqslant 1}=\partial_{x}\left(u^{2}\right)=\partial_{x}\left(X^{2}\right)+2 \partial_{x}\left(X u^{\geqslant 1}\right)+\partial_{x}\left(\left(u^{\geqslant 1}\right)^{2}\right) .
$$

Let us define the bilinear map

$$
B(f, g)=J \partial_{x}(f g)=\int_{0}^{\cdot} P{ }_{-s} \partial_{x}(f(s) g(s)) \mathrm{d} s .
$$

Then we can proceed by performing a further change of variables in order to remove the term $\partial_{x}\left(X^{2}\right)$ from the equation by setting

$$
u=X+B(X, X)+u^{\geqslant 2} \text {. }
$$

Now $u \geqslant 2$ satisfies

$$
\begin{aligned}
\mathscr{L} u^{\geqslant 2}= & 2 \partial_{x}(X B(X, X))+\partial_{x}(B(X, X) B(X, X)) \\
& +2 \partial_{x}\left(X u^{\geqslant 2}\right)+2 \partial_{x}\left(B(X, X) u^{\geqslant 2}\right)+\partial_{x}\left(\left(u^{\geqslant 2}\right)^{2}\right) .
\end{aligned}
$$

We can imagine to make a similar change of variables to get rid of the term

$$
2 \partial_{x}(X B(X, X))=\mathscr{L} 2 B(X, B(X, X)) .
$$

As we proceed in this inductive expansion, we generate a number of explicit terms, obtained by various combinations of $X$ and $B$. Since we will have to deal explicitly with at least some of these terms, it is convenient to represent them with a compact notation involving binary trees. A binary tree $\tau \in \mathcal{T}$ is either the root $\bullet$ or the combination of two smaller binary trees $\tau=\left(\tau_{1} \tau_{2}\right)$, where the two edges of the root of $\tau$ are attached to $\tau_{1}$ and $\tau_{2}$ respectively. For example

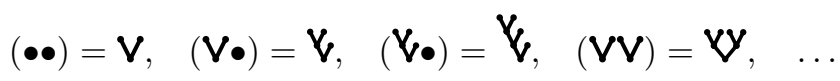

Then we define recursively

$$
X^{\bullet}=X, \quad X^{\left(\tau_{1} \tau_{2}\right)}=B\left(X^{\tau_{1}}, X^{\tau_{2}}\right),
$$

giving

$$
X^{\vee}=B(X, X), X^{\vee}=B\left(X^{\vee}, X\right), X^{\Downarrow}=B\left(X^{\vee}, X\right), X^{\vee \vee}=B\left(X^{\vee}, X^{\vee}\right),
$$

and so on. In this notation the expansion (6.2)-(6.3) reads

$$
\begin{gathered}
u=X+X^{\mathbf{V}}+u^{\geqslant 2}, \\
u^{\geqslant 2}=2 X^{\nvdash}+X^{\nvdash}+2 B\left(X, u^{\geqslant 2}\right)+2 B\left(X^{\mathbf{V}}, u^{\geqslant 2}\right)+B\left(u^{\geqslant 2}, u^{\geqslant 2}\right) .
\end{gathered}
$$


Remark 14. We observe that formally the solution $u$ of SBE can be expanded as an infinite sum of terms labelled by binary trees:

$$
u=\sum_{\tau \in \mathcal{T}} c(\tau) X^{\tau}
$$

where $c(\tau)$ is a combinatorial factor counting the number of planar trees which are isomorphic (as graphs) to $\tau$. For example $c(\bullet)=$ $1, c(\mathbf{V})=1, c(\mathbf{V})=2, c(\mathbf{V})=4, c(\mathbf{V})=1$ and in general $c(\tau)=\sum_{\tau_{1}, \tau_{2} \in \mathcal{T}} \mathbb{I}_{\left(\tau_{1} \tau_{2}\right)=\tau} c\left(\tau_{1}\right) c\left(\tau_{2}\right)$. Alternatively, we may truncate the summation at trees of degree at most $n$ and set

$$
u=\sum_{\tau \in \mathcal{T}, d(\tau)<n} c(\tau) X^{\tau}+u^{\geqslant n},
$$

where we denote by $d(\tau) \in \mathbb{N}_{0}$ the degree of the tree $\tau$, given by $d(\bullet)=0$ and then inductively $d\left(\left(\tau_{1} \tau_{2}\right)\right)=1+d\left(\tau_{1}\right)+d\left(\tau_{2}\right)$. For example $d(\mathbf{V})=1$, $d(\boldsymbol{V})=2, d(\mathbf{V})=3, d(\boldsymbol{V})=3$. We then obtain for the remainder

$$
\begin{aligned}
& u^{\geqslant n}=\sum_{\substack{\tau_{1}, \tau_{2}: d\left(\tau_{1}\right)<n, d\left(\tau_{2}\right)<n \\
d\left(\left(\tau_{1} \tau_{2}\right)\right) \geqslant n}} c\left(\tau_{1}\right) c\left(\tau_{2}\right) X^{\left(\tau_{1} \tau_{2}\right)} \\
& +\sum_{\substack{\tau: d(\tau)<n\\
}} c(\tau) B\left(X^{\tau}, u^{\geqslant n}\right)+B\left(u^{\geqslant n}, u^{\geqslant n}\right) .
\end{aligned}
$$

Our aim is to control the truncated expansion under the natural regularity assumptions in the white noise case, $X \in C \mathscr{C}^{-1 / 2-}$. Since (6.6) contains the term $B\left(X, u^{\geqslant n}\right)$ which in turn contains the paraproduct $J \partial_{x}\left(u^{\geqslant n} \prec X\right)$, the remainder $u^{\geqslant n}$ will be at best in $C \mathscr{C}^{1 / 2-}$. But then the sum of the regularities of $X$ and $u^{\geqslant n}$ is negative, and the term $B\left(X, u^{\geqslant n}\right)$ is not well defined. We therefore continue the expansion up to the point (turning out to be $u^{\geqslant 3}$ ) where we can set up a paracontrolled ansatz for the remainder, which will allow us to make sense of $\partial_{x}\left(X \circ u^{\geqslant n}\right)$ and thus of $B\left(X, u^{\geqslant n}\right)$.

\subsection{Paracontrolled solution}

Inspired by the partial tree series expansion of $u$ we set up a paracontrolled ansatz of the form

$$
u=X+X^{\vee}+2 X^{\vee}+u^{Q}, \quad u^{Q}=u^{\prime} \prec Q+u^{\sharp},
$$

where the functions $u^{\prime}, Q$ and $u^{\sharp}$ are for the moment arbitrary, but we assume $u^{\prime}, Q \in \mathscr{L}^{\gamma}$ and $u^{\sharp} \in \mathscr{L}^{2 \gamma}$, where from now on we fix 
$\gamma \in(1 / 3,1 / 2)$. For such $u$, the nonlinear term takes the form

$$
\begin{gathered}
\partial_{x} u^{2}=\partial_{x}\left(X^{2}+2 X^{\mathbf{V}} X+\left(X^{\mathbf{V}}\right)^{2}+4 X^{\vee} X\right)+2 \partial_{x}\left(u^{Q} X\right) \\
+2 \partial_{x}\left(X^{\mathbf{V}}\left(u^{Q}+2 X^{\vee}\right)\right)+\partial_{x}\left(\left(u^{Q}+2 X^{\vee}\right)^{2}\right),
\end{gathered}
$$

which gives us an equation for $u^{Q}$ :

$$
\begin{aligned}
& \mathscr{L} u^{Q} \\
& =\partial_{x}\left(\left(X^{\vee}\right)^{2}+4 X^{\vee} X\right)+2 \partial_{x}\left(u^{Q} X\right)+2 \partial_{x}\left(X^{\vee}\left(u^{Q}+2 X^{\natural}\right)\right)+\partial_{x}\left(\left(u^{Q}+2 X^{\vee}\right)^{2}\right) \\
& =\mathscr{L} X^{\vee \vee}+4 \mathscr{L} X^{\Downarrow}+2 \partial_{x}\left(u^{Q} X\right)+2 \partial_{x}\left(X^{\vee}\left(u^{Q}+2 X^{\Downarrow}\right)\right)+\partial_{x}\left(\left(u^{Q}+2 X^{\nvdash}\right)^{2}\right) \text {. }
\end{aligned}
$$

In Lemma 1 we showed that $X \in C H^{-1 / 2-}$. But now we understand Besov spaces and Gaussian hypercontractivity well enough so that we can return to the proof and modify the argumentation in order to show that $X \in$ $C \mathscr{C}^{-1 / 2-}$. If we then formally apply the paraproduct estimate Theorem 4 (which is of course not possible since the regularity requirements for the resonant term are not satisfied), we obtain $X^{2} \in C \mathscr{C}^{-1-}$ and then $\partial_{x} X^{2} \in C \mathscr{C}^{-2-}$. Therefore, $X^{\vee}=J\left(\partial_{x} X^{2}\right)$ should be in $C \mathscr{C}^{0-}$. Note that Lemma 11 does not apply here, because $-2-$ is not in $(-2,0)$. But we only needed this requirement to control the temporal regularity in $L^{\infty}$ of the image of $J$. For arbitrary $\alpha \in \mathbb{R}$ we have $J u \in C \mathscr{C}^{\alpha}$ whenever $u \in C \mathscr{C}^{\alpha-2}$, see for example Lemma A.9 in [14]. Similarly we derive the formal regularities of the remaining driving terms: $X^{\nvdash} \in \mathscr{L}^{1 / 2-}$, $X^{\nvdash} \in \mathscr{L}^{1 / 2-}$, and $X^{\nvdash} \in \mathscr{L}^{1-}$. In terms of $\gamma$, we can encode this as

$$
X \in C \mathscr{C}^{\gamma-1}, \quad X^{\vee} \in C \mathscr{C}^{2 \gamma-1}, \quad X^{\vee} \in \mathscr{L}^{\gamma}, \quad X^{\Downarrow} \in \mathscr{L}^{\gamma}, \quad X^{\nvdash} \in \mathscr{L}^{2 \gamma} .
$$

Under these regularity assumptions the term $2 \partial_{x}\left(X^{\mathbf{V}}\left(u^{Q}+X^{\vee}\right)\right)+\partial_{x}\left(\left(u^{Q}+\right.\right.$ $\left.\left.X^{\vee}\right)^{2}\right)$ is well defined and the only problematic term in (6.9) is $\partial_{x}\left(u^{Q} X\right)$. Using the paracontrolled structure of $u^{Q}$, we can make sense of $\partial_{x}\left(u^{Q} X\right)$ as a bounded operator provided that $Q \circ X \in C \mathscr{C}^{2 \gamma-1}$ is given. In other words, the right hand side of (6.9) is well defined for paracontrolled distributions.

Next, we should specify how to choose $Q$ and which form $u^{\prime}$ will take for the solution $u^{Q}$. We have formally

$$
\begin{aligned}
& \mathscr{L} u^{Q} \\
= & \mathscr{L} X^{\vee \vee}+4 \mathscr{L} X^{\nvdash}+2 \partial_{x}\left(u^{Q} X\right)+2 \partial_{x}\left(X^{\vee}\left(u^{Q}+2 X^{\nvdash}\right)\right)+\partial_{x}\left(\left(u^{Q}+2 X^{\Downarrow}\right)^{2}\right) \\
= & 4 \partial_{x}\left(X^{\nvdash} X\right)+2 \partial_{x}\left(u^{Q} X\right)+C \mathscr{C}^{2 \gamma-2} \\
= & 4 X^{\nvdash} \prec \partial_{x} X+2 u^{Q} \prec \partial_{x} X+C \mathscr{C}^{2 \gamma-2},
\end{aligned}
$$


where we assumed that not only $\mathscr{L} X^{\Downarrow} \in C \mathscr{C}^{\gamma-2}$, but that $\partial_{x}\left(X^{\nvdash} \circ X\right) \in$ $C \mathscr{C}^{2 \gamma-1}$ (which implies $\mathscr{L} X^{\Downarrow} \in C \mathscr{C}^{\gamma-2}$, but also the stronger statement $\left.\mathscr{L} X^{\nvdash}-X^{\nvdash} \prec \partial_{x} X \in C \mathscr{C}^{2 \gamma-2}\right)$. By Theorem $7, u^{Q}$ is paracontrolled by $J\left(\partial_{x} X\right)$, and in other words we should set $Q=J\left(\partial_{x} X\right)$. The derivative $u^{\prime}$ of the solution $u^{Q}$ will then be given by $u^{\prime}=4 X^{\nvdash}+2 u^{Q}$.

Unlike for PAM, here we do not need to introduce a renormalization. This is due to the fact that we differentiate after taking the square: to construct $u^{2}$, we would have to subtract an infinite constant and formally consider $u^{\diamond 2}=u^{2}-\infty$, or at the level of the approximation $u_{n}^{2}-c_{n}$. But then

$$
\partial_{x} u^{\diamond 2}=\lim _{n \rightarrow \infty} \partial_{x}\left(u_{n}^{2}-c_{n}\right)=\lim _{n \rightarrow \infty} \partial_{x} u_{n}^{2}=\partial_{x} u^{2} .
$$

So we obtain the following description of the driving data for the stochastic Burgers equation.

Definition 7. (SBE-enhancement) Let $\gamma \in(1 / 3,1 / 2)$ and let

$$
\mathcal{X}_{\text {sbe }} \subseteq C \mathscr{C}^{\gamma-1} \times C \mathscr{C}^{2 \gamma-1} \times \mathscr{L}^{\gamma} \times \mathscr{L}^{2 \gamma} \times C \mathscr{C}^{2 \gamma-1} \times C \mathscr{C}^{2 \gamma-1}
$$

be the closure of the image of the map $\Theta_{\text {sbe }}: C\left(\mathbb{R}_{+}, C^{\infty}(\mathbb{T})\right) \rightarrow \mathcal{X}_{\text {sbe }}$ given by

$$
\Theta_{\text {sbe }}(\theta)=\left(X(\theta), X^{\vee}(\theta), X^{\vee}(\theta), X^{\nvdash}(\theta),\left(X^{\nvdash} \circ X\right)(\theta),(Q \circ X)(\theta)\right),
$$

where

$$
\begin{aligned}
X(\theta) & =J\left(\partial_{x} \theta\right), \\
X^{\mathbf{v}}(\theta) & =B(X(\theta), X(\theta)), \\
X^{\vee}(\theta) & =B\left(X^{\mathbf{v}}(\theta), X(\theta)\right), \\
X^{\vee \mathbf{v}}(\theta) & =B\left(X^{\mathbf{v}}(\theta), X^{\mathbf{V}}(\theta)\right), \\
Q(\theta) & =J\left(\partial_{x} X(\theta)\right) .
\end{aligned}
$$

We will call $\Theta_{\text {sbe }}(\theta)$ the $\mathrm{SBE}-$ enhancement of the driving distribution $\theta$. For $T>0$ we define $\mathcal{X}_{\text {sbe }}(T)=\left.\mathcal{X}_{\text {sbe }}\right|_{[0, T]}$ and we write $\|\mathbb{X}\|_{\mathcal{X}_{\text {sbe }}(T)}$ for the norm of $\mathbb{X}$ in the Banach space $C_{T} \mathscr{C}^{\gamma-1} \times C_{T} \mathscr{C}^{2 \gamma-1} \times \mathscr{L}_{T}^{\gamma} \times \mathscr{L}_{T}^{2 \gamma} \times$ $C_{T} \mathscr{C}^{2 \gamma-1} \times C_{T} \mathscr{C}^{2 \gamma-1}$. Moreover, we define the distance $d_{\mathcal{X}_{\text {sbe }}(T)}(\mathbb{X}, \mathbb{X})=$ $\|\mathbb{X}-\tilde{\mathbb{X}}\|_{\mathcal{X}_{\text {sbe }}(T)}$

For every $\mathbb{X} \in \mathcal{X}_{\text {sbe }}$, there is an associated space of paracontrolled distributions:

Definition 8. Let $\mathbb{X} \in \mathcal{X}_{\text {sbe. }}$. Then the space of paracontrolled distributions $\mathscr{D}^{\gamma}(\mathbb{X})$ is defined as the set of all $\left(u, u^{\prime}\right) \in C \mathscr{C}^{\gamma-1} \times \mathscr{L}^{\gamma}$ with

$$
u=X+X^{\vee}+2 X^{\nvdash}+u^{\prime} \prec Q+u^{\sharp},
$$


where $u^{\sharp} \in \mathscr{L}^{2 \gamma}$. For $T>0$ we define

$$
\|u\|_{\mathscr{D}_{T}^{\gamma}}=\left\|u^{\prime}\right\|_{\mathscr{L}_{T}^{\gamma}}+\left\|u^{\sharp}\right\|_{C_{T} \mathscr{C}^{2 \gamma}} .
$$

If $\tilde{\mathbb{X}} \in \mathcal{X}_{\text {sbe }}$ and $\left(\tilde{u}, \tilde{u}^{\prime}\right) \in \mathscr{D}^{\gamma}(\tilde{\mathbb{X}})$, then we also write

$$
d_{\mathscr{D}_{T}^{\gamma}}(u, \tilde{u})=\left\|u^{\prime}-\tilde{u}^{\prime}\right\|_{\mathscr{L}_{T}^{\gamma}}+\left\|u^{\sharp}-\tilde{u}^{\sharp}\right\|_{C_{T} \mathscr{C}_{T}^{2 \gamma}} .
$$

We now have everything in place to solve sBE driven by $\mathbb{X} \in \mathcal{X}_{\text {sbe }}$.

Theorem 9. Let $\gamma \in(1 / 3,1 / 2)$. Let $\mathbb{X} \in \mathcal{X}_{\text {sbe }}$, write $\partial_{x} \theta=\mathscr{L} X$, and let $u_{0} \in \mathscr{C}^{2 \gamma}$. Then there exists a unique solution $u \in \mathscr{D}^{\gamma}(\mathbb{X})$ to the equation

$$
\mathscr{L} u=\partial_{x} u^{2}+\partial_{x} \theta, \quad u(0)=u_{0},
$$

up to the (possibly finite) explosion time $\tau=\tau(u)=\inf \left\{t \geqslant 0:\|u\|_{\mathscr{D}_{t}^{\gamma}}=\right.$ $\infty\}>0$.

Moreover, $u$ depends on $\left(u_{0}, \mathbb{X}\right) \in \mathscr{C}^{2 \gamma} \times \mathcal{X}_{\text {sbe }}$ in a locally Lipschitz continuous way: if $M, T>0$ are such that for all $\left(u_{0}, \mathbb{X}\right)$ with $\left\|u_{0}\right\|_{2 \gamma} \vee$ $\|\mathbb{X}\|_{\mathcal{X}_{\text {sbe }}(T)} \leqslant M$, the solution $u$ to the equation driven by $\left(u_{0}, \mathbb{X}\right)$ satisfies $\tau(u)>T$, and if $\left(\tilde{u}_{0}, \tilde{\mathbb{X}}\right)$ is another set of data bounded in the above sense by $M$, then there exists $C(M)>0$ for which

$$
d_{\mathscr{D}_{T}^{\gamma}}(u, \tilde{u}) \leqslant C(M)\left(\left\|u_{0}-\tilde{u}_{0}\right\|_{2 \gamma}+d_{\mathcal{X}_{\text {sbe }}(T)}(\mathbb{X}, \tilde{\mathbb{X}})\right) .
$$

Proof. By definition of the term $\partial_{x} u^{2}$, the distribution $u \in \mathscr{D}^{\gamma}(\mathbb{X})$ solves (6.12) if and only if $u^{Q}=u-X-X^{\vee}-2 X^{\vee}$ solves

$\mathscr{L} u^{Q}=\mathscr{L} X^{\vee \bigvee}+4 \partial_{x}\left(X^{\vee} X\right)+2 \partial_{x}\left(u^{Q} X\right)+2 \partial_{x}\left(X^{\vee}\left(u^{Q}+2 X^{\Downarrow}\right)\right)+\partial_{x}\left(\left(u^{Q}+2 X^{\Downarrow}\right)^{2}\right)$

with initial condition $u^{Q}(0)=u_{0}$. This equation is structurally very similar to PAM (5.1) and can be solved using the same arguments, which we do not reproduce here.

For this result to be of any use we still have to show that if $\xi$ is the spacetime white noise, then there is almost surely an element of $\mathcal{X}_{\text {sbe }}$ associated to $\partial_{x} \xi$. While for PAM we needed to construct only one term, here we have to construct five terms: $X^{\vee}, X^{\vee}, X^{\vee}, X^{\vee} \circ X, Q \circ X$. For details we refer to [16]. Alternatively we can simply differentiate the extended data which Hairer constructed for the KPZ equation in Chapter 5 of [18].

The same approach allows us to solve the KPZ equation $\mathscr{L} h=$ $\left(\partial_{x} h\right)^{\diamond 2}+\xi$, and if we are careful how to interpret the product $w \diamond \xi$, then also the linear heat equation $\mathscr{L} w=w \diamond \xi$. In both cases the solution depends continuously on some suitably extended data that is constructed from $\xi$ in a similar way as described in Definition 7. Moreover, the formal links between the three equations that we discussed in Section 4.2 can be made rigorous. These results are included in [16]. 


\section{Bibliography}

[1] Hajer Bahouri, Jean-Yves Chemin and Raphael Danchin. Fourier analysis and nonlinear partial differential equations. Springer, Berlin, 2011.

[2] Guillaume Bal and $\mathrm{Yu} \mathrm{Gu}$. Limiting models for equations with large random potential; a review. Commun. Math. Sci., 13(3), 729-748, 2015.

[3] Lorenzo Bertini and Giambattista Giacomin. Stochastic Burgers and KPZ equations from particle systems. Comm. Math. Phys., 183(3):571-607, 1997.

[4] Jean-Michel Bony. Calcul symbolique et propagation des singularites pour les Équations aux dérivées partielles non linéaires. Ann. Sci. Éc. Norm. Supér. (4), 14:209-246, 1981.

[5] Terence Chan. Scaling limits of Wick ordered KPZ equation. Comm. Math. Phys., 209(3):671-690, 2000.

[6] Ivan Corwin. The Kardar-Parisi-Zhang equation and universality class. Random Matrices Theory Appl., 1(1), 2012.

[7] Pedro Echeverría. A criterion for invariant measures of Markov processes.Z. Wahrsch. Verw. Gebiete, 61(1):1-16, 1982.

[8] Peter Friz and Martin Hairer. A Course on Rough Paths. Springer, Berlin, 2014.

[9] Peter Friz and Nicolas Victoir. Multidimensional stochastic processes as rough paths. Theory and applications. Cambridge University Press, Cambridge, 2010.

[10] Patricia Gonçalves and Milton Jara. Universality of KPZ equation. ArXiv:1003.4478, 2010. 
[11] Patricia Gonçalves and Milton Jara. Nonlinear fluctuations of weakly asymmetric interacting particle systems. Arch. Ration. Mech. Anal., 212(2), 597-644, 2014.

[12] Massimiliano Gubinelli. Controlling rough paths. J. Funct. Anal., 216(1):86-140, 2004.

[13] Massimiliano Gubinelli. Ramification of rough paths. J. Differential Equations, 248(4):693-721, 2010.

[14] Massimiliano Gubinelli, Peter Imkeller and Nicolas Perkowski. Paracontrolled distributions and singular PDEs. Forum Math. Pi, 3(6), 2015.

[15] Massimiliano Gubinelli and Milton Jara. Regularization by noise and stochastic Burgers equations. Stochastic Partial Differential Equations: Analysis and Computations, 1(2):325-350, 2013.

[16] Massimiliano Gubinelli and Nicolas Perkowski. KPZ reloaded. ArXiv:1508.03877, 2015.

[17] Massimiliano Gubinelli and Nicolas Perkowski. Energy solutions of KPZ are unique. ArXiv:1508.07764, 2015.

[18] Martin Hairer. Solving the KPZ equation. Ann. Math., 178(2):559$664,2013$.

[19] Martin Hairer. A theory of regularity structures. Invent. math., 198(2):269-504, 2014.

[20] Martin Hairer, Etienne Pardoux and Andrey Piatnitski. Random homogenisation of a highly oscillatory singular potential. Stochastic Partial Differential Equations: Analysis and Computations, 1(4):571-605, 2013.

[21] Yaozhong Hu. Chaos expansion of heat equations with white noise potentials. Potential Anal., 16(1):45-66, 2002.

[22] Svante Janson. Gaussian Hilbert spaces, volume 129 of Cambridge Tracts in Mathematics. Cambridge University Press, Cambridge, 1997.

[23] Ioannis Karatzas and Steven Shreve. Brownian Motion and Stochastic Calculus. Springer-Verlag New York, 1998.

[24] Mehran Kardar, Giorgio Parisi and Yi-Cheng Zhang. Dynamic scaling of growing interfaces. Physical Review Letters, 56(9):889-892, 1986. 
[25] Terry J. Lyons. Differential equations driven by rough signals. Rev. Mat. Iberoam., 14(2):215-310, 1998.

[26] Terry J. Lyons, Michael Caruana and Thierry Lévy. Differential equations driven by rough paths, volume 1908 of Lecture Notes in Mathematics. Springer, Berlin, 2007.

[27] Terry J. Lyons and Zhongmin Qian. System control and rough paths. Oxford University Press, 2002.

[28] Jeremy Quastel. Introduction to KPZ. Current developments in mathematics, 2011, Int. Press, Somerville, MA, 2012.

[29] Jeremy Quastel and Herbert Spohn. The One-Dimensional KPZ Equation and Its Universality Class. J. Stat. Phys., 160(4):965-984, 2015.

[30] Francesco Russo, Pierre Vallois and Jochen Wolf. A generalized class of Lyons-Zheng processes. Bernoulli, 7(2):363-379, 2001.

[31] Hans-Jürgen Schmeisser and Hans Triebel. Topics in Fourier analysis and function spaces. Akademische Verlagsgesellschaft Geest \& Portig K.-G., Leipzig, 1987.

[32] Laurence C. Young. An inequality of the Hölder type, connected with Stieltjes integration. Acta Math., 67(1):251-282, 1936.

Massimiliano Gubinelli

CEREMADE \& CNRS UMR 7534, Université Paris Dauphine and IUF, France.

Hausdorff Center of Mathematics \& Institute of Applied Mathematics, Universität Bonn, Germany.

gubinelli@iam.uni-bonn.de

http://www.iam.uni-bonn.de/abteilung-gubinelli/home

Nicolas Perkowski

Institut für Mathematik

Humboldt-Universität zu Berlin, Germany.

perkowsk@math.hu-berlin.de

https://www.math.hu-berlin.de/ perkowsk/ 